UNITED STATES

DEPARTMENT OF THE INTERIOR

GEOLOGICAL SURVEY

\title{
ANALYSES OF ROCK AND STREAM-SEDIMENT SAMPLES FROM THE \\ PRINCE RUPERT QUADRANGLE, SOUTHEASTERN ALASKA
}

By R. D. Koch and R. L. Elliott

OPEN-FILE REPORT 78-156-B

This report is preliminary and has not been edited or reviewed for conformity with Geological Survey standards and nomenclature

Menlo Park, California 


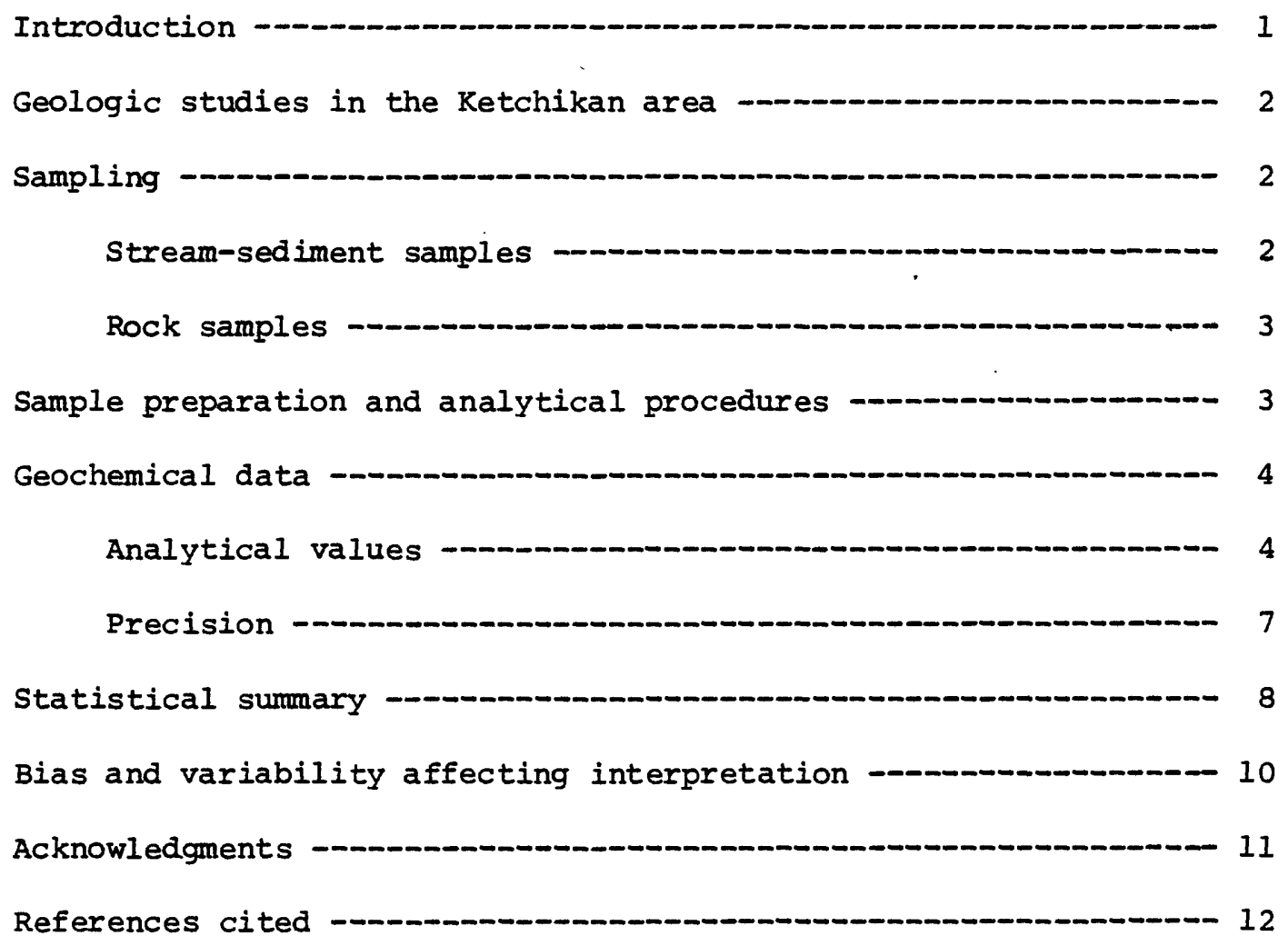

\section{Illustrations}

Plate I. Sample location maps

Figure 1. Stream-sediment sample locations

2. Rock sample locations

Tables

Table 1. Lower determination limits and units for analyses --- 5

2. Qualification codes

3. Class intervals of the six-step scale

4. Rock name and mineral modifier codes - 15

5. Statistical summary - stream-sediment samples - - 17

6. Analytical data for stream-sediment samples -_-_-_ 46

7. Statistical summary - rock samples - 61

8. Analytical data for rock samples 


\section{Analyses of rock and stream-sediment samples from Prince Rupert quadrangle, southeastern Alaska}

by

R. D. Koch and R. I. Elliott

\section{Introduction}

A reconnaissance geochemical sampling program was conducted between 1975 and 1977 in the Ketchikan and Prince Rupert 1:250,000-scale quadrangles, southeastern Alaska. The study was done to assist in a mineral resource evaluation of the area as part of the Alaska Mineral Resource Assessment Program (AMRAP). This report contains the analytical data for all samples collected in the Prince Rupert quadrangle during this study and also includes data from samples collected during U.S. Geological Survey mapping in 1969 and 1970. Data from 117 rock and 206 stream-sediment samples are reported. These samples comprise all of the normal rock and stream-sediment geochemical samples collected during U.S. Geological Survey geological mapping investigations within the Prince Rupert quadrangle through 1977. Brief statistical summaries of the rock and stream-sediment analytical data are included in this report.

Analytical data from geochemical sampling within the Ketchikan quadrangle directly north of the Prince Rupert quadrangle are contained in two companion reports (Koch and Elliott, 1978a, 1978b), and data from the southern portion of the Bradfield Canal quadrangle (north of the Ketchikan quadrangle) in Koch and others (1976). Data from all of the normal U.S. Geological Survey rock and stream-sediment geochemical samples collected in the Ketchikan and Prince Rupert quadrangles are available on magnetic computer tape (Koch, Van Trump, and McDanal, 1978). 


\section{Geologic studies in the Ketchikan area}

The northeastern portion of the Prince Rupert quadrangle is underlain by Cretaceous granitic rocks of the Coast Range metamorphic-plutonic complex and associated greenschist to amphiholite facies metasedimentary and metavolcanic rocks. A metamorphosed early Paleozoic or older trondhjemite pluton underlies the area between Foggy Bay and Cape Fox. To the west, Duke and Percy Islands are dominated by early Cretacenus ultramafic bodies which intrude metamorphosed sedimentary and volcanic rocks.

The earliest comprehensive discussions of the geology of the Ketchikan area are contained in reports by Wright and wright (1.908) and Buddington and Chapin (1929). Recent discussions of the geology of this area include a report by Hutchison (1970) on the Coast Range metamorphic-plutonic complex in the Prince Rupert region of British Columbia and one by Irvine (1974) on the ultramafic complex on Duke Island.

Recent geologic investigations by the U.S. Geological Survey in the Prince Rupert quadrangle began with reconnaissance mapping in the Prince Rupert D2 and D3 quadrangles in 1969 and 1970. Field studies continued from 1975 to 1977 (Berg, Elliott, and Köch, 1976; Elliott, Smith, and Hudson, 1976; Koch and others, 1977). Reconnaissance mapping of the Ketchikan and Prince Rupert quadrangles has been completed and a geologic map at a scale of $1: 250,000$ has been published (Berg and others, 1978).

\section{Sampling}

Stream-sediment Samples

Stream-sediment samples were generally collected from the finest, most organic-free sediment in the active stream channel. In rare instances where this was not possible, samples were collected from bank or terrace 
deposits adjacent to the channel. At sites below the tree line, it was not always possible to collect a sample completely free of organic material and a smail number of samples have low to occasionally high organic content. Stream-sediment samples collected from shoreline sites were obtained above highest high tide level whenever possible. Most of the study area is steep and sediment in the resulting swift streams is essentially all detrital material resulting from mechanical, not chemical weathering. The bulk of most stream-sediment samples comprises material ranging in size from very fine sand to pebbles. Samples with a large percentage of silt- and claysized material are rare and are generally from areas of low elevation and gèntle gradient.

Rock Samples

The rock samples are primarily grab samples chosen to provide data on background values for a lithologic unit. The majority of these samples are representative of the dominant lithologies at the sample site. A lesser number of samples were collected from minor lithologies, known mineralized occurrences, or outcrops that are conspicuously iron-stained or contain visible metallic minerals.

\section{Sample preparation and analytical procedures}

Stream-sediment samples were dried and sieved; the -80 mesh $(-0.2 \mathrm{~mm})$ fraction was pulverized and a split was analyzed. Rock samples were crushed, a split was pulverized and a split of this material analyzed.

Samples were analyzed by the Branch of Exploration Research of the U.S. Geological survey for up to 30 elements by the six-step semiquantitative emission spectrographic method (Grimes and Marranzino, 1968), and for gold, copper, lead and zinc by atomic absorption spectrophotometry (Ward and 
others, 1969). Some analyses were not performed on all samples. The semiquantitative spectrographic analyses were performed by E. F. Cooley, K. J. Curry, and C. L. Forn. The atomic absorption analyses were done by D. E. Deira, J. D. Hoffman, A. L. Meier, R. L. Miller, R. M. O'Leary, J. A. Roybal, and R. F. Sanzolone.

\section{Geochemical data}

Locations of sample sites are shown on plate I and identificd by station numbers. The analytical data for the stream-sediment and rock samples are given in tables 6 and 8 respectively and are identified by station number with a letter appended to the station number to distinguish different samples from the same station. Four stream-sediment samples were re-anaiyzed as part of a test of analytical variance. These sample numbers appear twice-in table 6 along with data for both analyses:

Eàch rock sample has been assigned a rock type and is labeled in table 8 with a rock name code consisting of a two-letter rock-name symbol which may be followed by one or more two-letter mineral-modifier symbols. An explanation of these rock-name and mineral-modifier codes is listed in table 4 (p. 15).

\section{Analytical Values}

Analytical results are reported in tables 6 and 8 as percent of the sample (for spectrographic analyses of $\mathrm{Fe}, \mathrm{Mg}, \mathrm{Ca}$, and $\mathrm{Ti}$ ) or as parts per million (ppm) for all other analyses. The minimum limits of determination and the units used to report each analysis are listed in table 1.

A single-letter symbol is used by USGS analysts to indicate that no analysis was performed for an element or that the analytical result is outside the limits of determinability. These symbols (commonly called 
Table 1. Lower determination limits and units for analyses performed from 1969 through 1977. S - indicates spectrographic analysis, AA - indicates atomic absorption analysis. The units used to report values for each analytical procedure are listed after the determination limit.

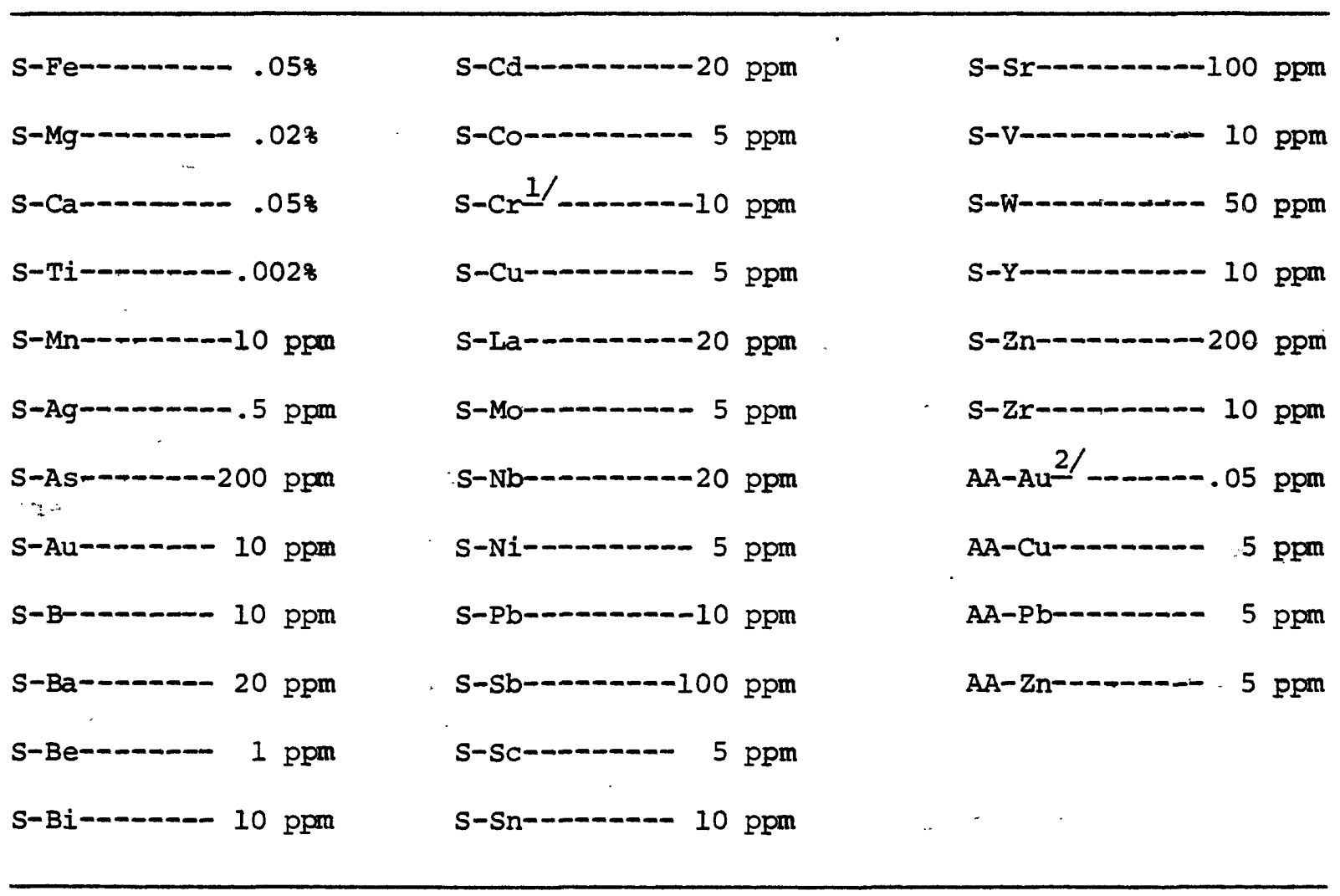

I/ 5 ppm prior to 1970 .

2/. .02 ppm prior to 1972 . 
"qualification codes") are used in the statistical sumary but some are represented differently in the data tables. An explanation of both forms is listed in table 2 . The code $T$ does not appear in these data.

Table 2.--Qualification codes

\begin{tabular}{|c|c|c|}
\hline $\begin{array}{l}\text { Qualification } \\
\text { code }\end{array}$ & $\begin{array}{l}\text { Form in } \\
\text { tables } 6 \& 8\end{array}$ & Explanation \\
\hline B & - & No analytical data for this analysis. \\
\hline $\mathrm{N}$ & $\mathbf{N}$ & Nothing detected by analysis. \\
\hline L & $<$ & $\begin{array}{l}\text { Element detected but below listed } \\
\text { value (lower limit of determinability). }\end{array}$ \\
\hline G & $>$ & $\begin{array}{l}\text { Element detected in amount greater } \\
\text { than listed value (upper limit of } \\
\text { determinability). }\end{array}$ \\
\hline $\mathrm{H}$ & $($ value $=0)$ & Interference - no valid data. \\
\hline$T$ & & Trace \\
\hline
\end{tabular}

Because the original computer printout is used in the tables, element: symbols are in capital letters; for example, the symbol for iron, $\mathrm{Fe}$, is shown as FE, magnesium, Mg, is shown as MG, and so on. In the tables, theprefix S stands for spectrographic analysis and AA for atomic absorption analysis.

Results from semiquantitative emission spectrographic analyses (also referred to as six-step spectrographic analyses) are reported as the midpoints of geometric class intervals. Midpoints of class intervals and the associated class interval boundaries are listed in table 3 . Reported values may be any integral power of ten times one of the listed class interval midpoints. 
Table 3. Class intervals of the six-step scale.

\section{Class interval midpoint}

$\begin{array}{llc}1.0 & 0.83 & 1.2 \\ 1.5 & 1.2 & 1.8 \\ 2.0 & 1.8 & 2.6 \\ 3.0 & 2.6 & 3.8 \\ 5.0 & 3.8 & 5.6 \\ 7.0 & 5.6 & 8.3 \\ 10.0 & 8.3 & 12.0\end{array}$

Precision

Tests have been performed to determine the analytical precision of the six-step semiquantitative spectrographic technique used by the Branch of Exploration Research (Motooka and Grimes, 1976). These tests indicate that the frequency with which values from repeated analyses of the same sample will fall within the class interval containing the "true" value (as measured by the mean of a series of analytical runs) plus or minus one and two adjoining intervals is approximately 83 percent and 96 percent respectively. For example, if a value is reported as 3.0 , the probability is .83 that a repeated analysis would be reported as $2.0,3.0$, or 5.0 . This study found analytical variance to be consistent for a variety of geologic materials, and to show no appreciable difference between elements or concentration ranges except near the limits of determinability. Another experiment (Johnson and others, 1977) suggests that analytical precision may vary appreciably between elements. Analyses by the atomic absorption methods are not reported on the six-step scale; they are more sensitive and more precise than spectrographic analyses. 


\section{Statistical summary}

The analytical data from the stream-sediment and rock samples were processed by a computer program known as GEOSUM and the program's output is presented in tables 5 and 7 respectively. For samples which have been re-analyzed, the values listed second in the data tables have been arbitrárily omitted from the statistical summary to reduce bias. The GEOSUM program is designed to summarize and tabulate geochemical data--primarily data from semiquantitative spectrographic analyses. All distributions are treated in terms of the six-step class intervals described above and thus the atomic absorption data are regrouped to fit into these intervals. The program output consists of: (a) a frequency distribution table, histogram, sumary of qualified values, range of values anä geometric mean and deviàtion for each element, and (b) a statistical summary for all elements, which includes geometric means and geometric deviations.

The histograms are on a logarithmic scale and are computed using the class intervals of the six-step semiquantitative scale. The histogram bars are composed of X's; each X represents approximately $I$ percent of the total number of samples analyzed for that element. Decimal numbers are printed by the computer as powers of 10, for example:

$$
\begin{array}{lllll}
7.0 \mathrm{e}-01 & \text { means } & 7.0 \times 10^{-1} & \text { or } & 0.7 \\
7.0 \mathrm{e}+00 & \text { means } & 7.0 \times 10^{\circ} & \text { or } & 7.0 \\
7.0 \mathrm{e}+01 & \text { means } & 7.0 \times 10^{1} & \text { or } & 70.0 \\
7.0 \mathrm{e}+02 & \text { means } & 7.0 \times 10^{2} & \text { or } & 700.0
\end{array}
$$

The frequency distribution tables, histograms, and statistics for each element were derived using only data values within the range of analytical determination which have been used since 1975. Between 1969 and 1975, the lower limits of determinability were raised for atomic absorption analyses 
of Au and for spectrographic analyses of Cr. Unqualified values less than the current determinability limits and all values qualified with $\mathrm{N}, \mathrm{L}$, $G$, or $\mathrm{H}$ were ignored in these computations. The resulting frequency tables and statistics are biased and the histograms incomplete.

The summaries at the ends of tables 5 and 7 show which elements have qualified values, as well as the number of values having each type of qualification. The summary also presents a recomputed geometric mean and geometric deviation using a method devised by A. J. Cohen for treating censored distributions. If an element has no qualified data values, the mean and geometric deviation will be the same in both this summary and on the page within the table for the particular element. For elements with qualified data, the estimates of mean and geometric deviation are unbiased in a strict sense only where the data are derived from a log-normal parent population, but experiments have shown that large departures from this requirement do not usually invalidate the results. Acceptance and use of the estimates, however, is the responsibility of the user.

The geometric mean is the antilogarithm of the arithmetic mean of the logarithms of the analyses. It is not an estimate of geochemical abundance but of "central tendency" (or characteristic value) for a frequency distribution that is approximately symetrical on a logarithmic scale. The geometric mean is useful for characterizing many geochemical distributions. The geometric deviation is the antilogarithm of the standard deviation of the logarithms of the analyses.

For further discussion of geometric mean and geometric deviation and of Cohen's method for censored distributions, see Miesch $(1963,1967)$. 


\section{Bias and variability affecting interpretation}

In reviewing the data in tables 6 and 8 and the statistical summary in tables 5 and 7 , several sources of bias and variability in the data must be considered. Factors including time limitations, weather, snow and vegetative cover, outcrop exposure, and availability of helicopter landing sites prevented uniform sampling in all areas. Uneven sample density also resulted from more concentrated sampling of some areas which show evidence of mineralization such as iron-staining or visible metallic minerals. This practice has biased the data slightly in favor of samples containing values above true background levels. This applies most strongly to rock samples. For stream-sediment samples the requirement of truly random sampling--that all potential samples have an equal likelihood of being selected--is not met either. In addition, samples were collected from a large area, where lithologic units of various origins or rock types may comprise several dissimilar geochemical populations. No attempt has been made here to group samples on the basis of geological or geochemical affinity. The summary of their values thus provides only a general indication of the trends that may be present.

Values from stream-sediment samples which contain appreciable organic material may be influenced by scavenging. Variability of any value is influenced by many factors including the difficulty of obtaining representative samples of inhomogeneous media, variation in sample preparation and variability inherent in the analytical techniques. It is likely with any large data-set that errors have occurred during recording, key-punching, and editing of the data which have gone undetected. Therefore, high values for a single element or a single site should be considered questionable indicators of bedrock mineralization. 


\section{Acknowledgments}

We wish to thank several U.S. Geological Survey colleagues for their considerable cooperation and assistance. George Van Trump, Jr., provided invaluable aid by resolving computer-programming problems. Steven $\mathrm{K}$. McDanal and Christine M. McDougal helped locate and collect computer data-files from previous projects and with entry of data into the computer. Michael F. Diggles worked tirelessly proofing enormous lists and cluttered maps.

We also wish to thank Kenneth Eichner of TEMSCO Helicopters who was most generous with his time and advice and volunteered logistical assistance which greatly aided our field efforts. The skillful flying of TEMsCo pilot, Barry Roberts, allowed us to sample this particularly inaccessible terrain. Bud Bodding, captain of M/N MYTIME, went out of his way to help with our fieldwork and guided us safely through several field seasons. 


\section{References cited}

Berg, H. C., Elliott, R. L., and Koch, R. D., 1976, Progress report on geology and mineral resources of the Ketchikan quadrangle, in Cobb, E. H., ed., The U.S. Geological Survey in Alaska; accomplishments during 1975: U.S. Geol. Survey Circ. 733, p. 62-64.

Berg, H. C., Elliott, R. L., Smith, J. G., and Koch, R. D., 1978, Geologic map of the Ketchikan and Prince Rupert quadrangles, Alaska: U.S. Geol. Survey open-file rept. 78-73A, 1 sheet, scale 1:250,000.

Buddington, A. F., and Chapin, T., 1929, Geology and mineral deposits of southeastern Alaska: U.S. Geol. Survey Bull. 800, 398 p.

Elliott, R. L., Smith, J. G., and Hudson, Travis, 1976, Upper Tertiary highlevel plutons of the Smeaton Bay area, southeastern Alaska: U.S. Geol. Survey open-file rept. 76-507, $15 \mathrm{p}$.

Grimes, D. J., and Marranzino, A. P., 1968, Direct-current arc and alternating-current spark emission spectrographic field methods for the semiquantitative analysis of geologic materials: U.S. Geol. Survey Circ. 591, 6 p.

Hutchison, W. W., 1970, Metamorphic framework and plutonic styles in the Prince Rupert region of the Central Coast Mountains, British Columbia: Canadian Jour. Earth Sci., v. 7, p. 376-405.

Irvine, T. N., 1974, Petrology of the Duke Island Ultramafic Complex, southeastern Alaska: Geol. Soc. America Mem. 138, 240 p.

Johnson, B. R., Forn, C. I., Hoffman, J. D., Brew, D. A., and Nutt, C. J., 1977, Geochemical sampling of stream sediments, Tracy Arm, southeastern Alaska, in Blean, K. M., ed., The United States Geological Survey in Alaska, 1976: U.S. Geol. Survey Circ. 751-B, p. B80-B82. 
Koch, R. D., and Elliott, R. L., 1978a, Analyses of stream-sediment samples from the Ketchikan quadrangle, southeastern Alaska: U.S. Geol. Survey open-file rept. $78-156 \mathrm{C}, 214 \mathrm{p}$. 1978b, Analyses of rock samples from the Ketchikan quadrangle, southeastern Alaska: U.S. Geol. Survey open-file rept. 78-156A, 163 p. Koch, R. D., Elliott, R. L., Berg, H. C., and Smith, J. G., 1976, Analyses of rock and stream-sediment samples from the Bradfield Canal quadrangle, southeastern Alaska: U.S. Geol. Survey open-file rept. 76-486, 136 p. Koch, R. D., Elliott, R. L., Smith, J. G., and Berg, H. C., 1977, Metamorphosed trondhjemite of the Alexander terrane in Coast Range plutonic complex, in Blean, K. M., ed., The United States Geological Survey in Alaska; accomplishments during 1976: U.S. Geol. Survey Circ. 751-B, p. B72-B74.

Koch, R. D., Van Trump, George, Jr., and McDanal, S. K., 1978, Magnetic tape containing analytical data for rock and stream-sediment samples from Ketchikan and Prince Rupert quadrangles, southeastern Alaska: U.S. Geol. Survey Rept., 8 p., computer tape [Available from the Natl. Tech. Inf. Service, U.S. Dept. Commerce, Springfield, VA, NTIS PB-276-7771. Motooka, J. M., and Grimes, D. J., 1976, Analytical precision of one-sixth order semiquantitative spectrographic analysis: U.S. Geol. Survey Circ. 738,25 p.

Miesch, A. T., 1963, Distribution of elements in Colorado Plateau uraniumdeposits--a preliminary report: U. S. Geol. Survey Bull. 1147-E, 57 p. 1967, Methods of computation for estimating geochemical abundance: U.S. Geol. Survey Prof. Paper 574-B, 15 p.

Ward, F. N., Nakagawa, H. M., Harms, T. F., and Van Sickle, G. H., 1969, Atomic-absorption methods of analysis useful in geochemical exploration: U.S. Geol. Survey Bull. 1289, 45 p. 
Wright, F. E., and Wright, C. W., 1908, The Ketchikan and Wrangell mining districts, Aláska: U.S. Geol. Survey Bull. 347, 210 p. 


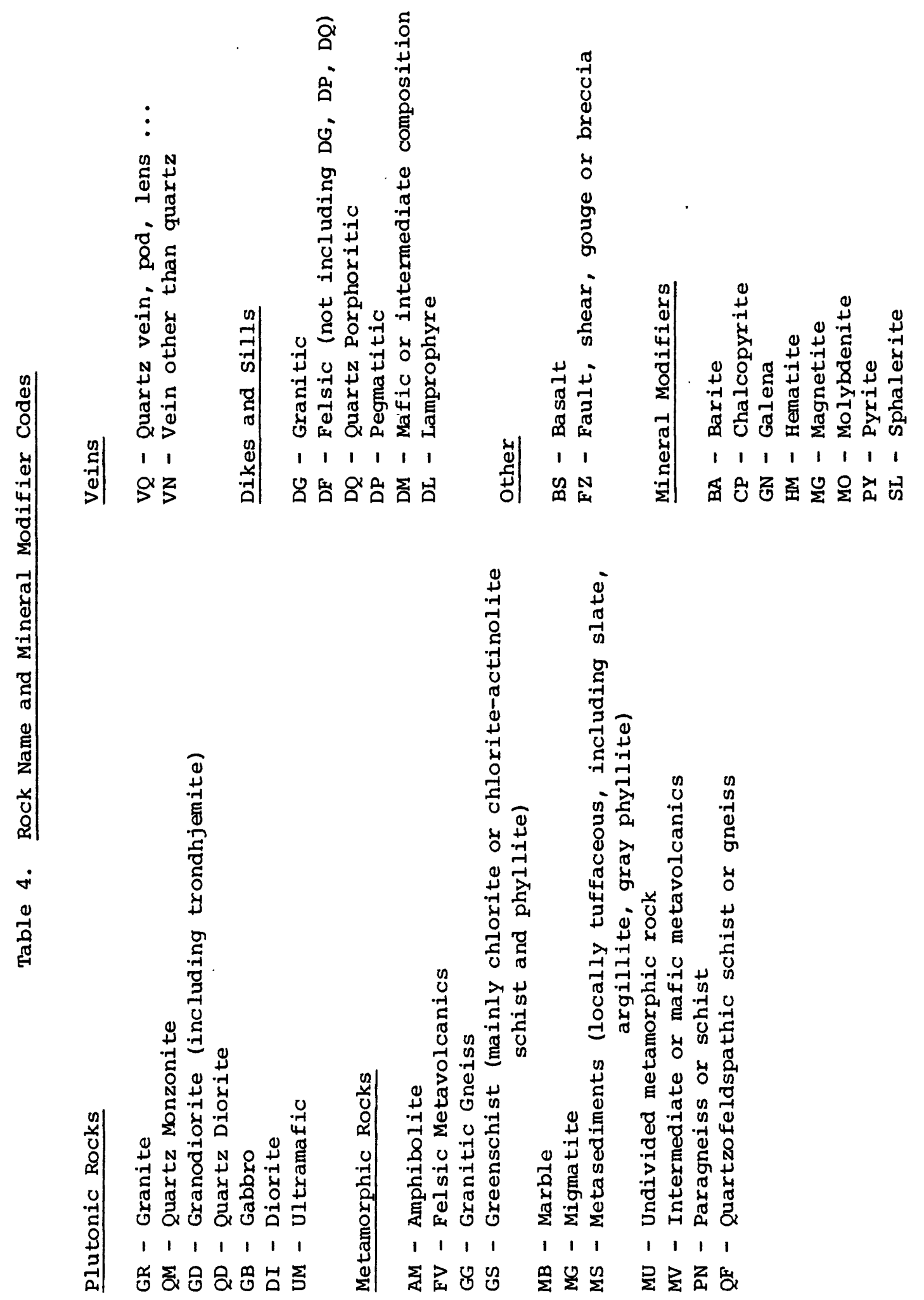




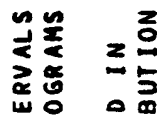

แ응

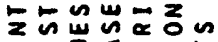

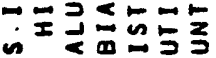

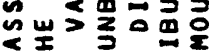

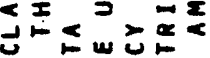

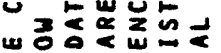

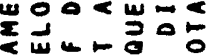

的向出出

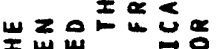

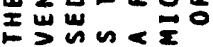

- $0 \leq w \omega$

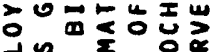

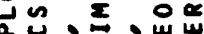

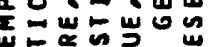

$5 \div 0 د$ 운ㄷㄴ

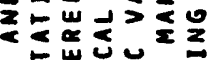

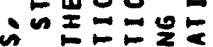

แ⿻上丨纹

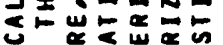

的出的巨出

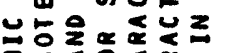

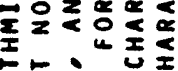

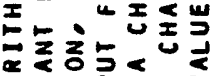

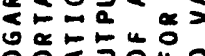

웅중의

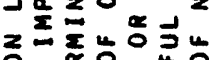

잉

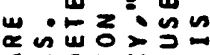

< w口出 wo

舟,

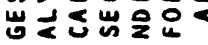

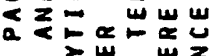

ט

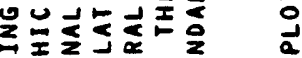

후인

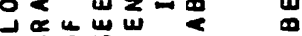

可过台出岂是

웅 $n$.

w元出。

I

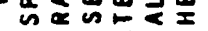

중w

nII

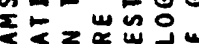

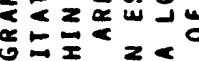

O픈

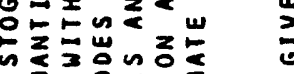

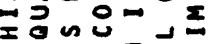

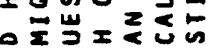

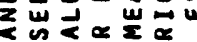

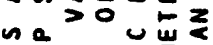

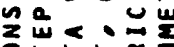

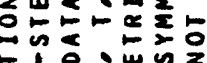

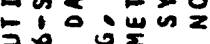

D 00

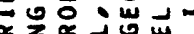

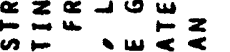

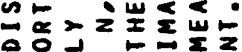

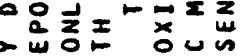

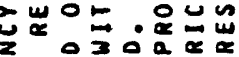

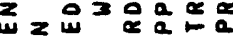

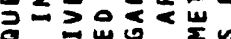

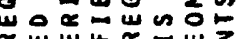

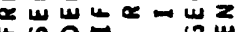

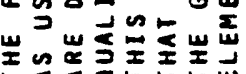

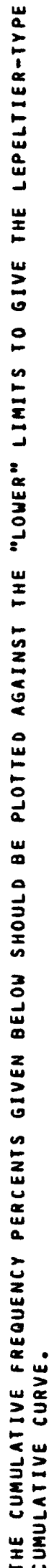



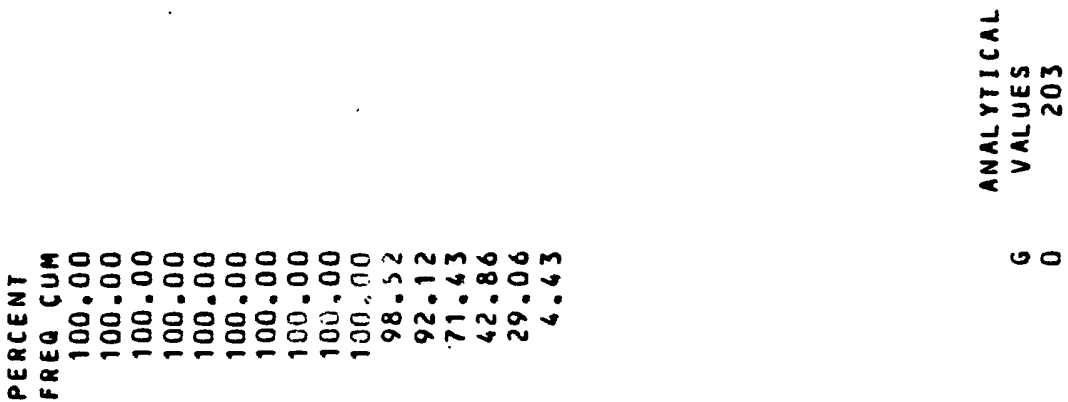

$00 \%$ w 웅ㅇㅇㅇㅇㅇㅇㅇㅇㅇㅛ แ⿺辶巛

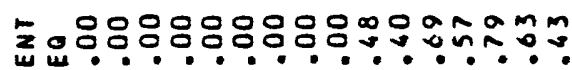
ú00000000-000m: a

疍

$=\quad$ i $000000000 m m n \infty i \infty n$

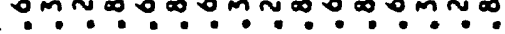

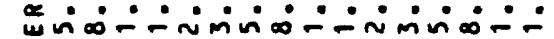
$\approx \frac{a}{5}$

$\Xi_{1}, 1,1,1,1,1,1,1,1,1$

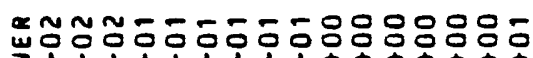
눙! ! ! ! ! ! ! ! ! t t t t t t $\perp \infty \circ m \sim \infty 0_{\infty} \circ m \sim \infty 0_{\infty} 0 m \sim$

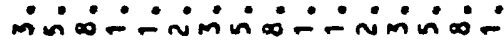

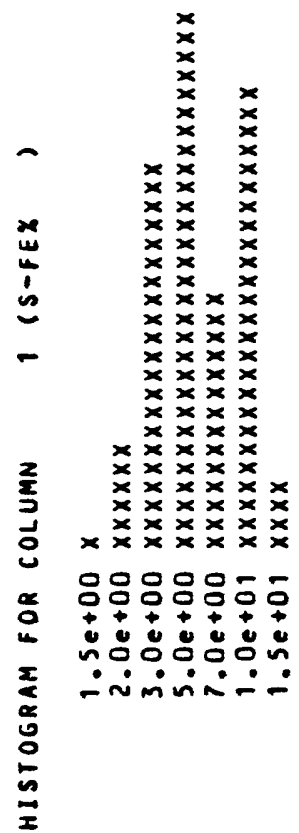

-0:

$\infty m$

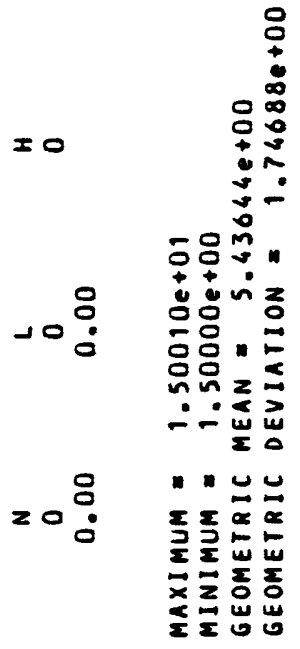




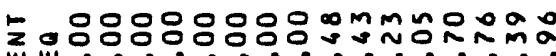

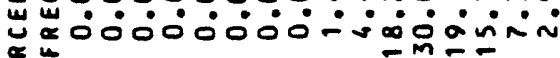
w

- $\stackrel{8}{\circ}$

픈

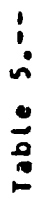

$\sim$

\& $00000000 m a n g 0 m \tilde{m}$

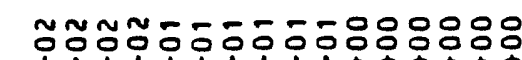
:

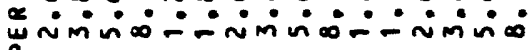
$\because \frac{a}{a}$

$\Sigma_{1}, 1,1,1,1,1,1,1$

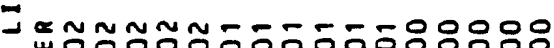
눙우웅우웅ㅇㅇㅇㅇㅇㅇㅇㅇ은

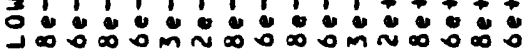
-

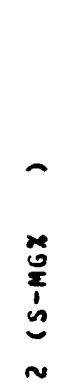

m m

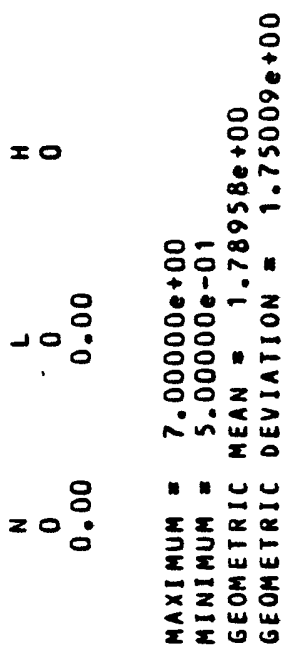




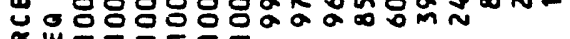
w

远

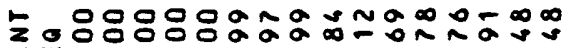

$-0 \stackrel{8}{0}$

$4 \times 000000-00 \approx 0 \leq n$

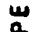

is

$\underset{\sim}{\infty}$

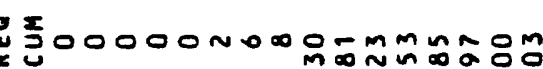

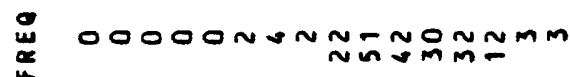

$$
\text { NẼ }
$$

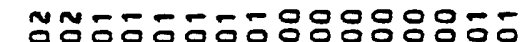

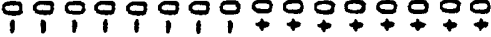

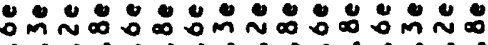
世 $\sim \frac{a}{a}$

$1,1,1,1,1,1,1,1$

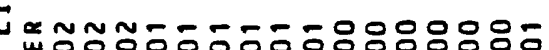

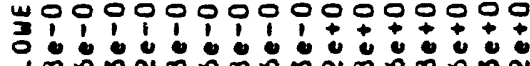

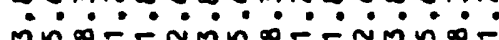

$\infty m$

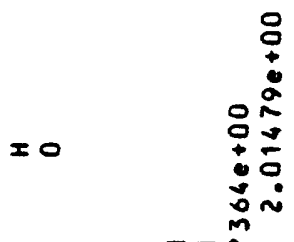
ธธ㇒ก

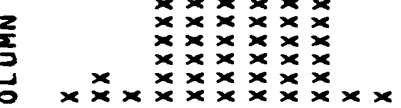

듣ㅇㅇㅇㅇㅇㅇㅇㅇ읃

-

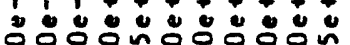

$t ! \dot{0}=$ +0 ○

ㅇㅇㅇ 용요은 m min-๐nmmi-:

$$
20 \stackrel{\circ}{\circ}
$$

*

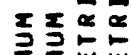
现 Хरํํㅇㅎㅇ 玉灭岕 
z u

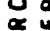

总

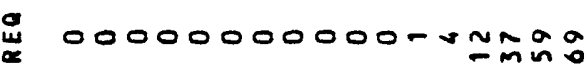

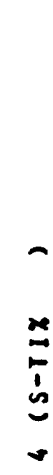

-

$\infty m$

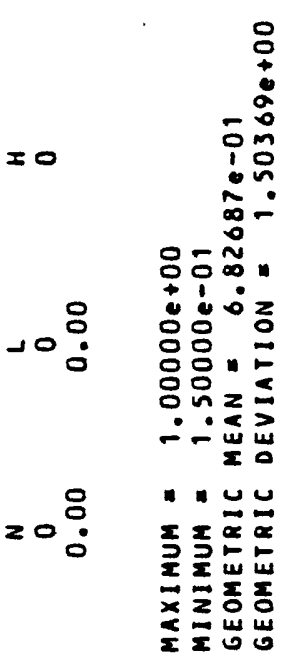




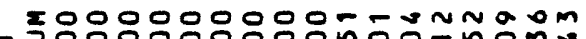

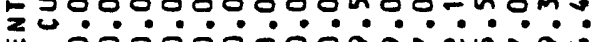

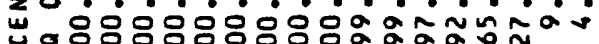

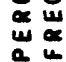

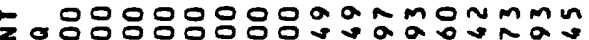
z

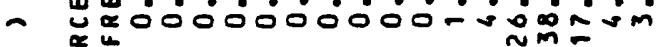
a

$\frac{z}{\vdots}$

元

i

n

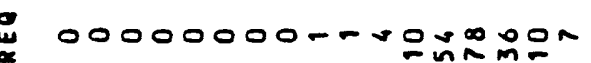

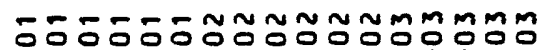

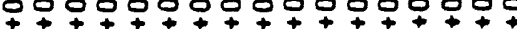

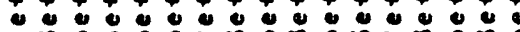
ก⿻:丷a $\cong \frac{a}{5}$

$1,1,1,1,1,1,1,1,1$

×

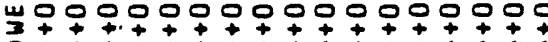

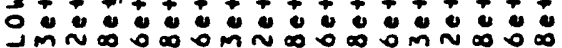
$\because \simeq \infty 0 \infty 0 m \sim \infty 0 \infty 0 m \sim \infty 0 \infty$

- $\stackrel{0}{0}$
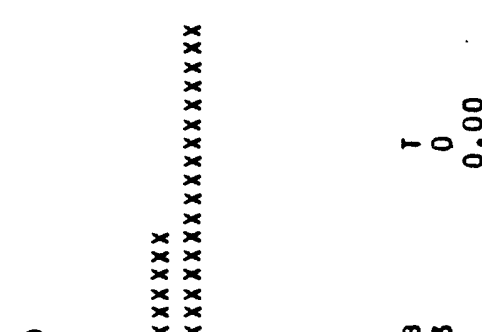

$\infty m$

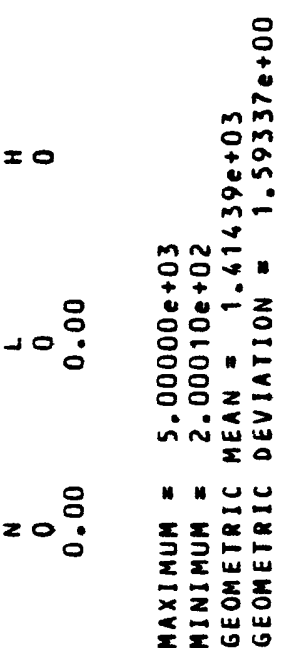




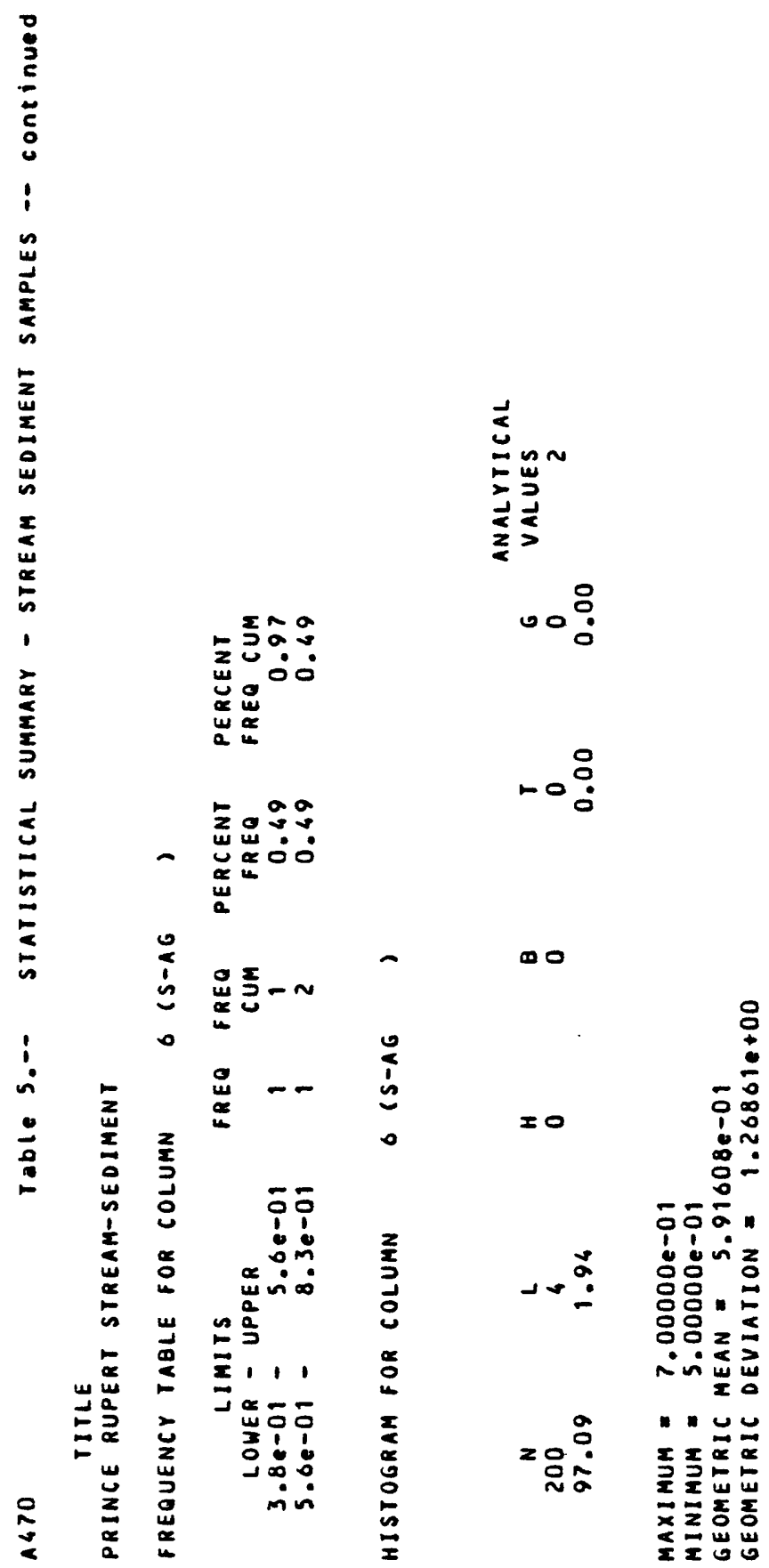


w $\infty^{\infty} \infty+\infty$

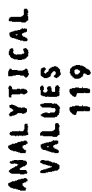

$$
\text { ○ }
$$

- 80

zommiñog แ

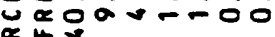
$\div-$

a.

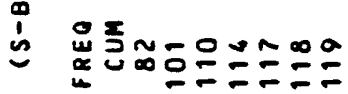
$a$

!

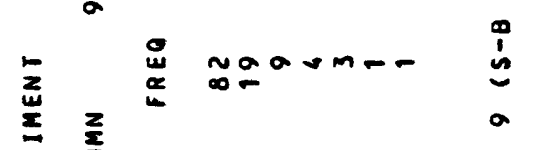

$x$
$x$
$x$
$x$
$x$
$x$
$x$
$x$
$x$
$x$
$x$
$x$
$x$
$x$
$x$
$x$
$x$
$x$
$x$
$x$
$x$
$x$
$x$
$x$
$x$
$x$
$x$
$x$
$x$

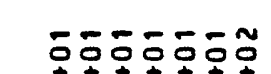

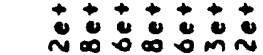

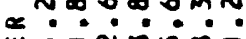
w- - Nmina

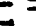

1111111

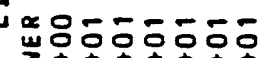

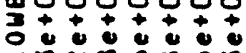

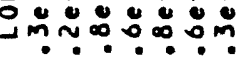

$\infty m$

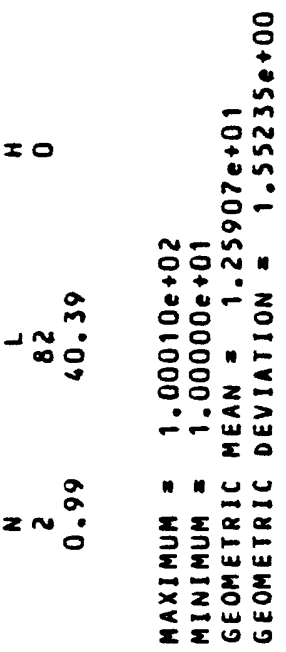




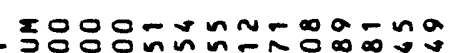

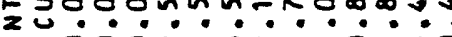

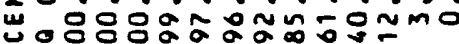

a d

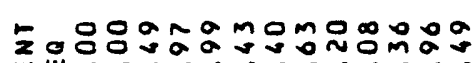
ứ 运

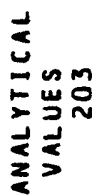

.

-0: 总 : oorvamosna-

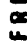

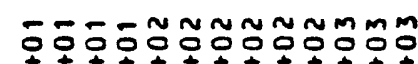
:

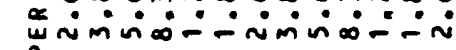

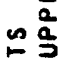
$=$ $\vec{\Sigma}, 1,1, \ldots, 1$, มะร 눙우웅우우운우웅웅

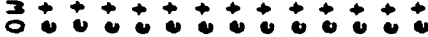
- imini- - iminio
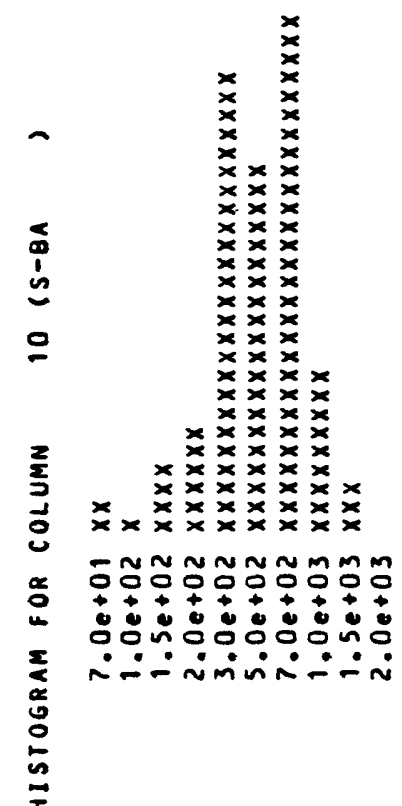

$\infty m$

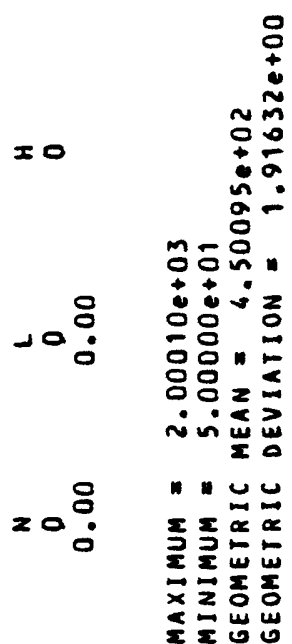




$$
\begin{array}{r}
008 \\
0 \\
-0 \frac{0}{0}
\end{array}
$$

은요웅

ư

正品

a

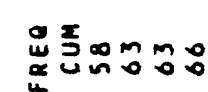

$E$

!:

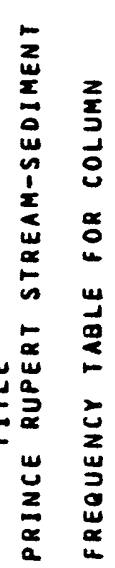

« \#nom

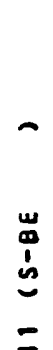

$x$
$x$
$x$
$x$
$x$
$x$
$x$

mo

㗭

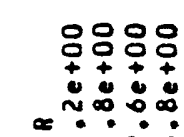

aa

⿷匚1,1

뚣ㅇㅇㅇㅇㅇㅇㅇ 뜽연연

\lrcorner $m \sim \infty$

$\dot{\infty}-\dot{\sim}$

$=$

2.

- 옹ㅇㅇㅇㅇㅇ

-

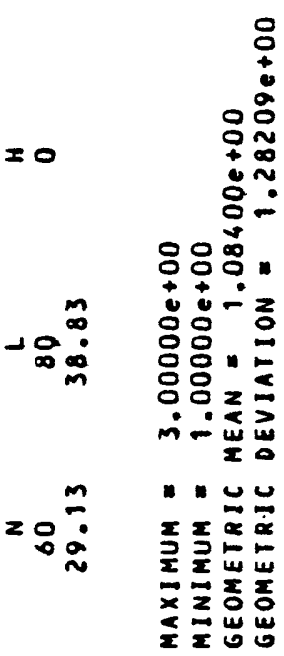




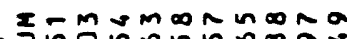

ร

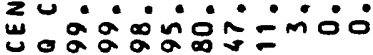

$\sum_{0}^{\frac{2}{2}}$

w

5
$\vdots$
$\vdots$
$\vdots$

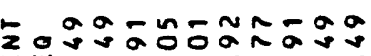

m

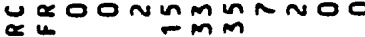
แ.

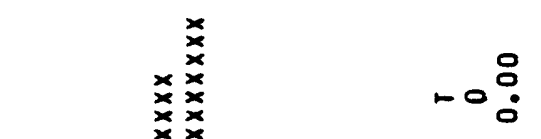

is

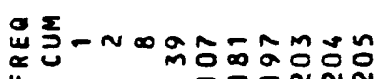

:

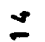

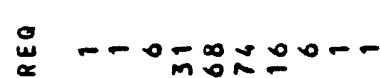

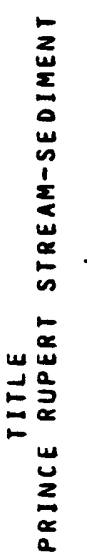

$\stackrel{\circ}{u}$

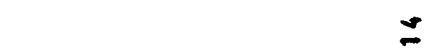

응응ㅎㄷㅇㄷㅇㅇㅇㅇ

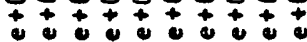
$\because m \sim \infty 0 \infty 0 m \sim \infty$

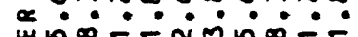
$\sim \frac{u}{a}$

ש

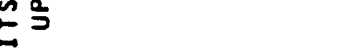

1111111111

풍ㅇㅇㅇㅇㅁㄷㅎㅎㅎㅎㅇ

ํ. : t: $-\infty \circ m \sim \infty 0 \infty 0 m \sim$ in $\dot{0} \dot{-}-\dot{n} \dot{m} \dot{0} \dot{\infty}-\dot{-}$

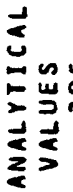

용

$-0 \stackrel{8}{\circ}$

$\infty$

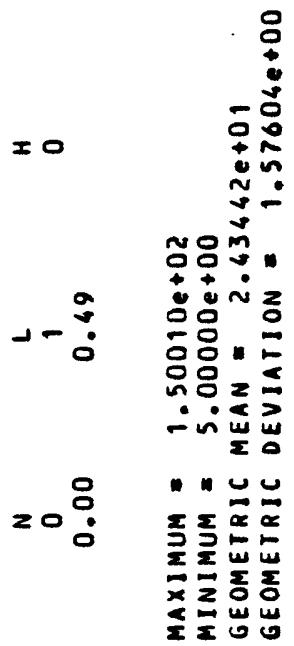




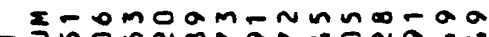

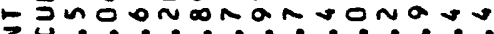

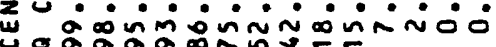
兽 แै山 a

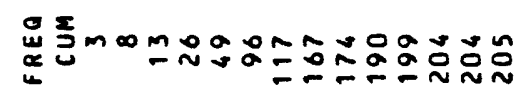
:

․ mnnmmñoñonor $\underset{4}{4}$

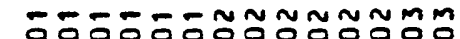
tot? $\sim$

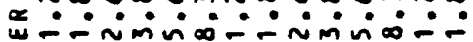

$n \frac{a}{a}$

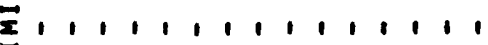

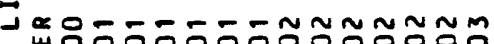

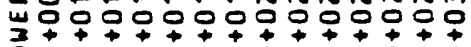

ㄱㄴ :- :

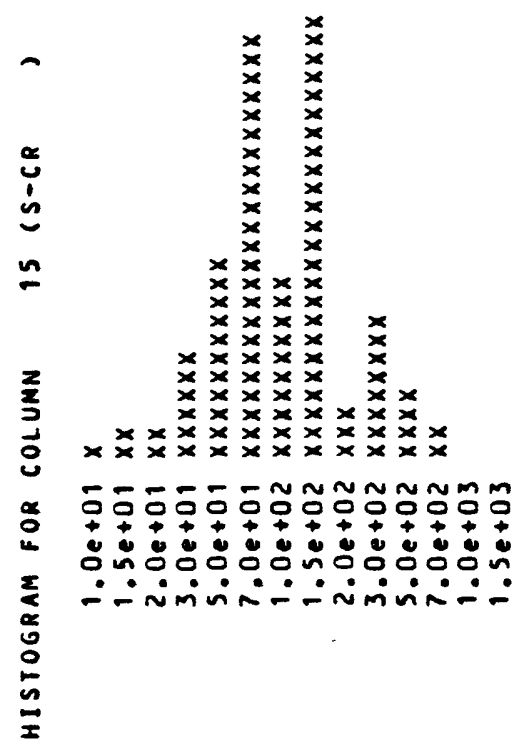

$\infty 0$

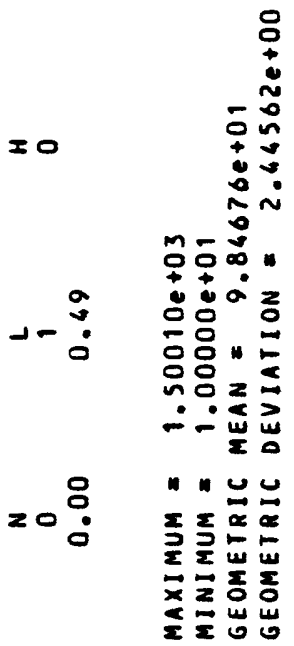


z

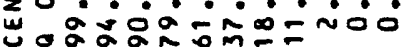
崖

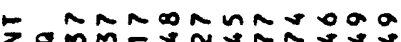
zе

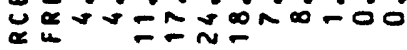
$\omega$

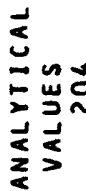

ט 0

$-0 \stackrel{\circ}{\circ}$

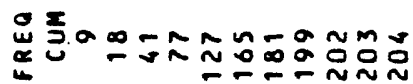

:

a $00 m 00000$.

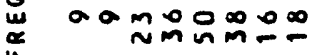

응듀듀융ํำ

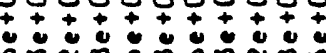
ดับัณ:

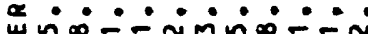
$n a$

$\approx 5$

工 $11,111,1,1,111$

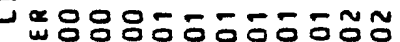

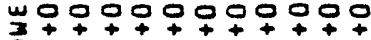

定: $\dot{1} \dot{0}=\dot{\sim} \dot{\sim} \dot{\infty} \dot{-}$

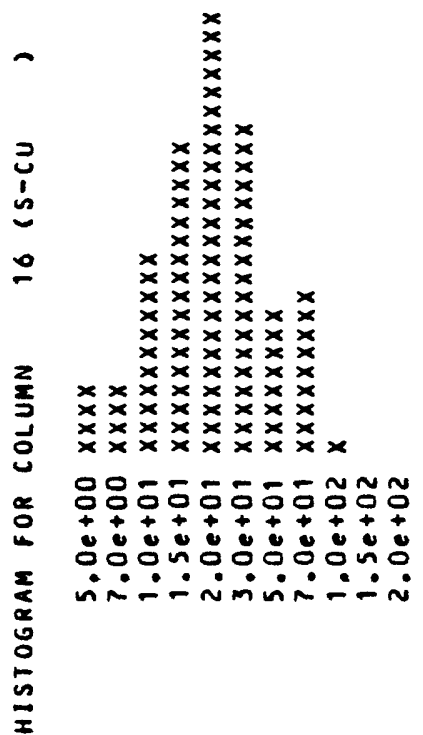

$\infty$

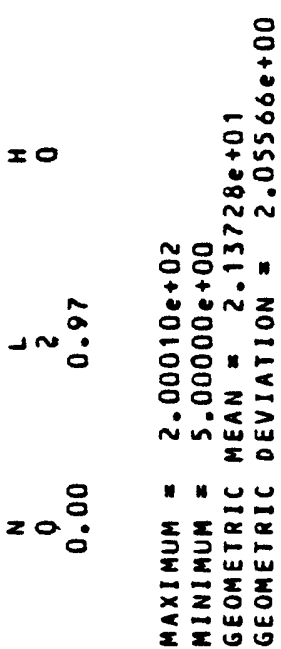


In $\infty=m-\infty \leq a+a$

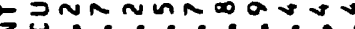

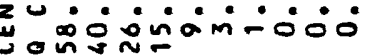
西果

$w \approx$

בै

뜬

至

ט 0

- $0 \stackrel{8}{0}$

- $0 \infty m m=0000$

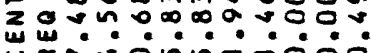
我 a

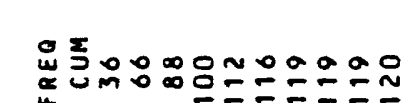

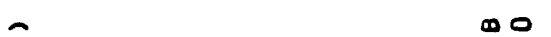

i

$\simeq$

:

is

$\underset{x}{x} \times \underset{x}{x}$

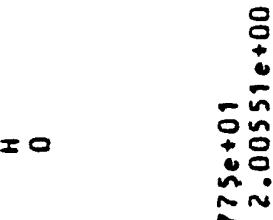

ㄷํㅇ.

- 등ํํำกั $+t+t+t+t+t$

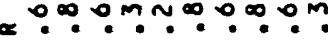

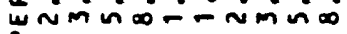
$\simeq \frac{a}{2}$

$\sum 1: 1111,1,1$,

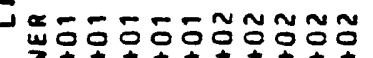

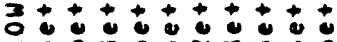

$\pm 0 \infty 0 m \sim \infty 0 \infty$

- imis- - imi

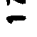

$x \times x$

$x \times x$

$x \times$

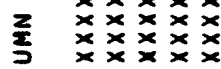

$\begin{array}{ll}x \times \times x \times x \times x & x \times x \\ x & x \times 4\end{array}$

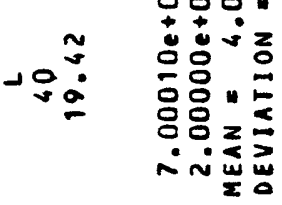

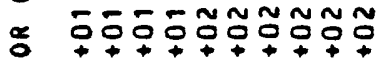

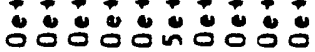

$=\underset{\sim}{m}$

* $\div \div$

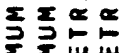

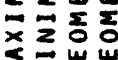

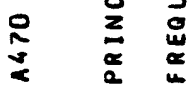




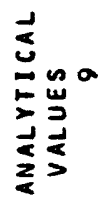

- InMn

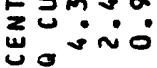

ט 0

ㅇ․

w

-0

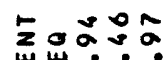

u世

的

a

䍃

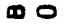

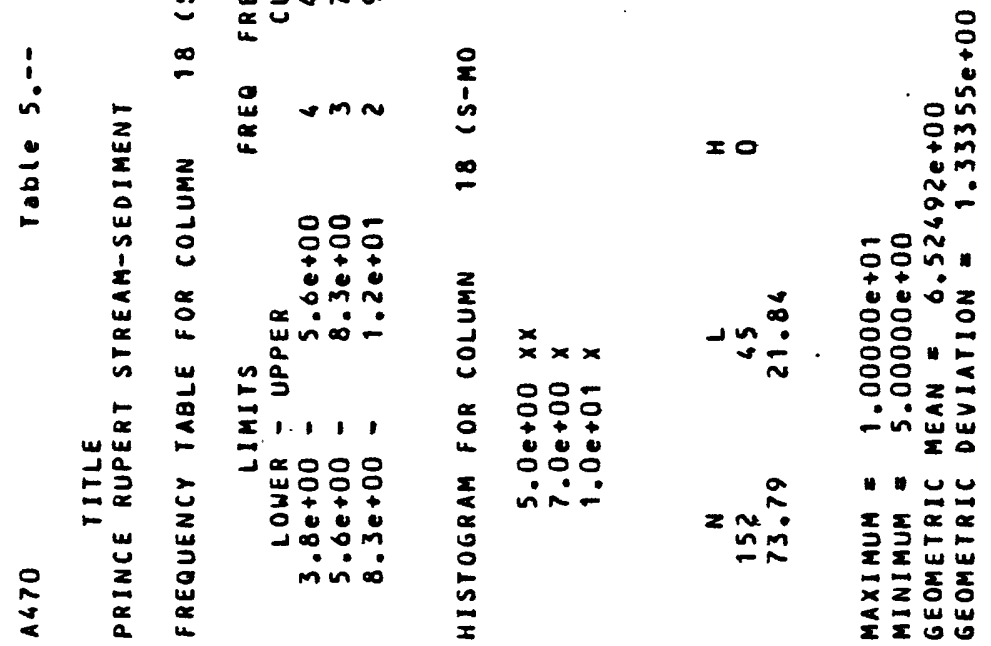


$\sum_{w}^{2}$

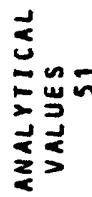

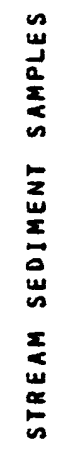

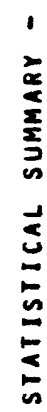

- 0 :

- $0 \stackrel{8}{\circ}$

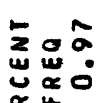

W

品

幽

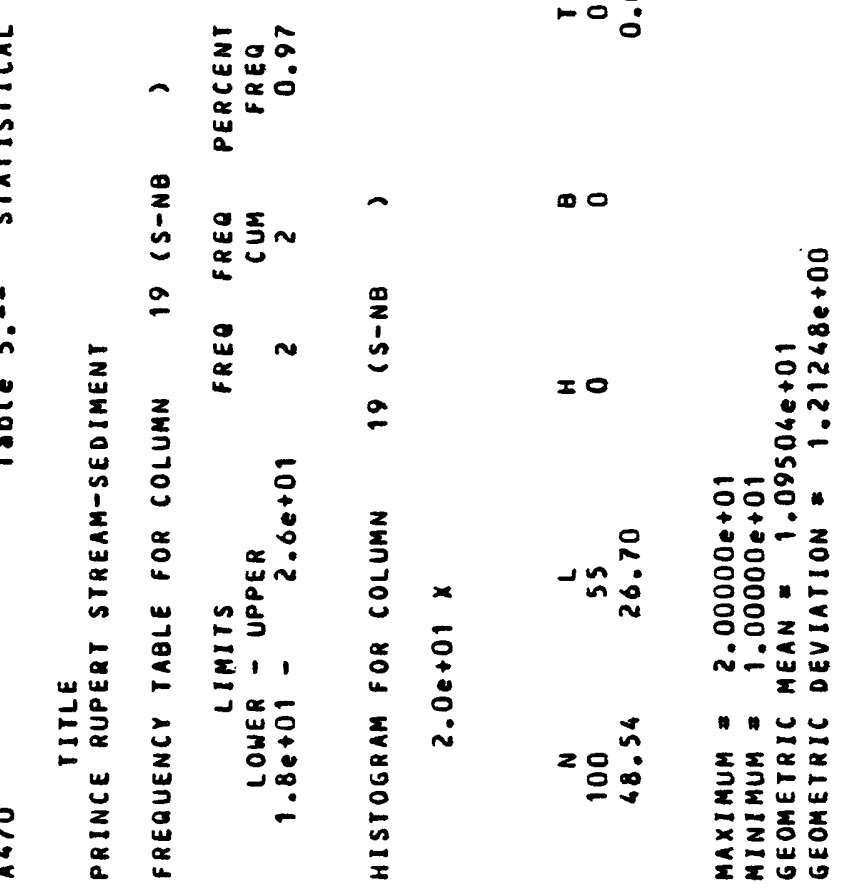


ร

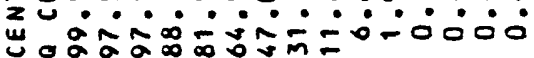

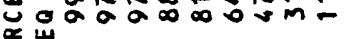
$\stackrel{m}{a}$

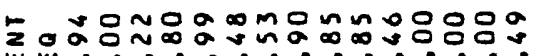

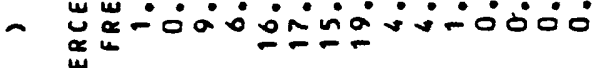
a

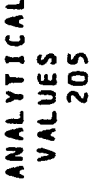

ง

-

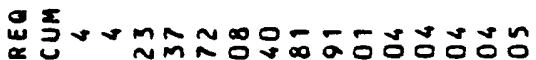
$\stackrel{\circ}{\sim}$

นMก

总 og:

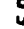

$\sum_{0}^{2}$

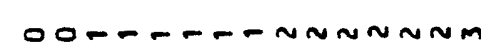

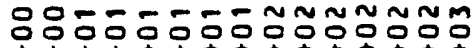

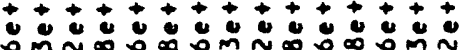
:m a $\sim \frac{a}{a}$

₹ $1,1,1,1,1,1,1,1,1,1,1,1,1,1,0$

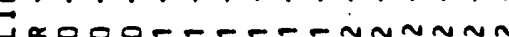

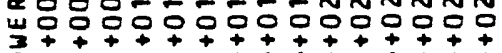

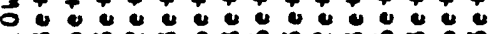

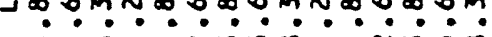

$\infty 0$

\section{2
$\vdots$
$\vdots$
0}
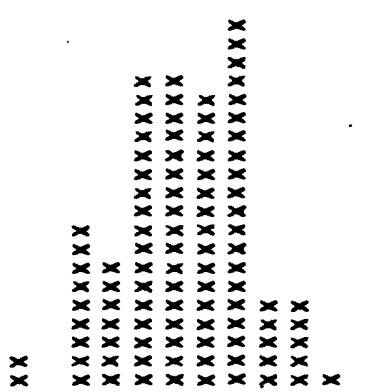

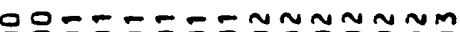

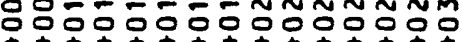
:

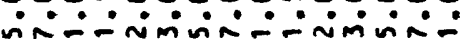

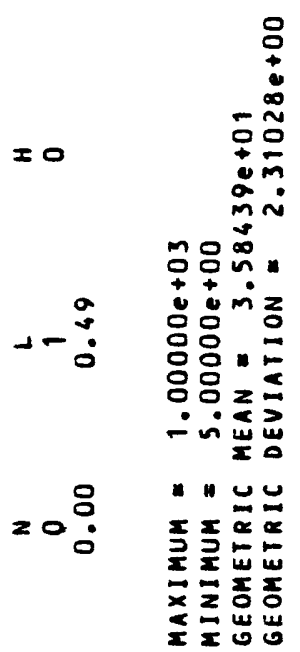


Eoñ

- ய

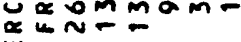
a

a

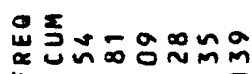
iv

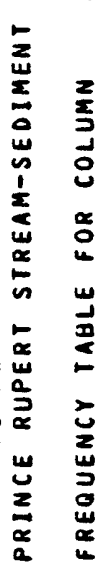

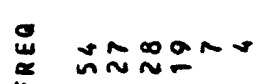

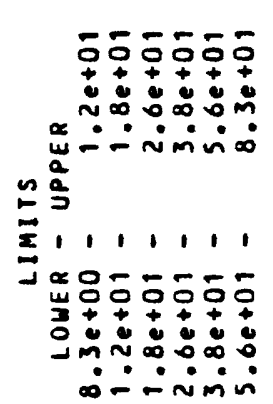

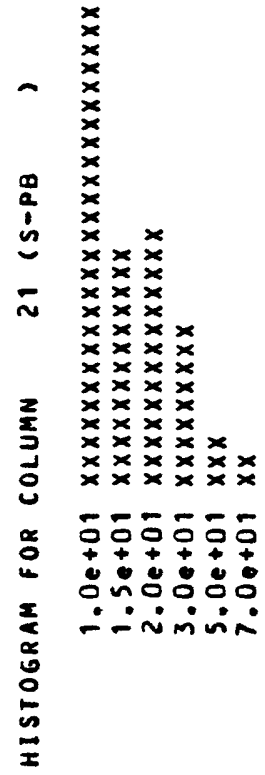

$\infty 0$

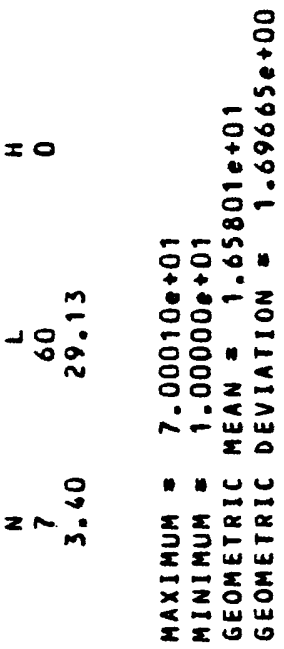


연?ำง

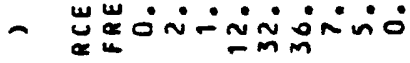
w

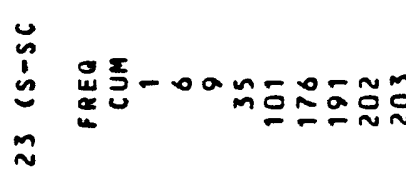

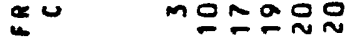

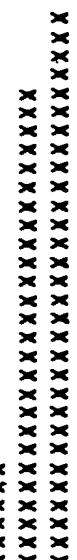

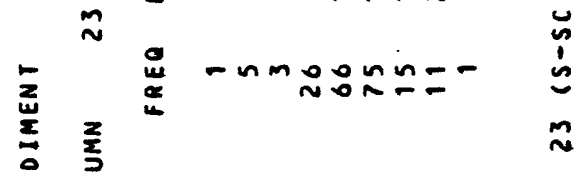
$+t+t+t+t+$ $0 m \sim \infty 0 \infty 0 m \sim$

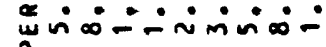
a

\section{z $\quad x \times x \times x$}

$x x x x$

$x \times \underset{x}{x} \times \underset{x}{x}$

응훙ㅎㅇㅇ

E, 1, 1, 1, 1

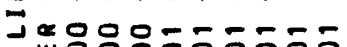

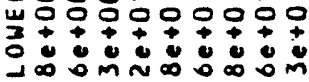
$-\infty 0 m \sim \infty 0 \infty \cdots$

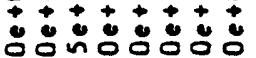
$\because \because$ nivin-

$\infty m$

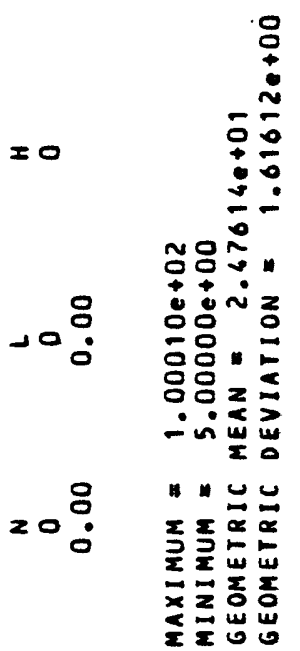




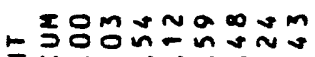

z

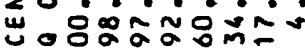

幽

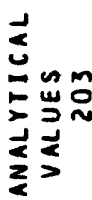

৩

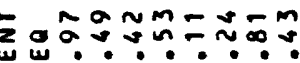

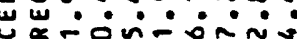

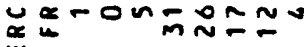

a.

$\underset{x}{x}$

-

$\infty m$

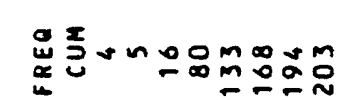

:

แ

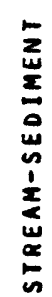

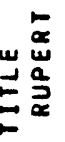

군

ํํํํํํํํำ :

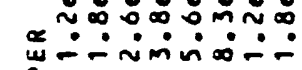

$\cong \stackrel{a}{\frac{a}{5}}$

1111111

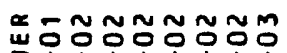

$3^{+}+t+t+t+$

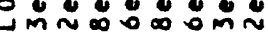

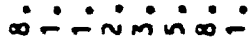
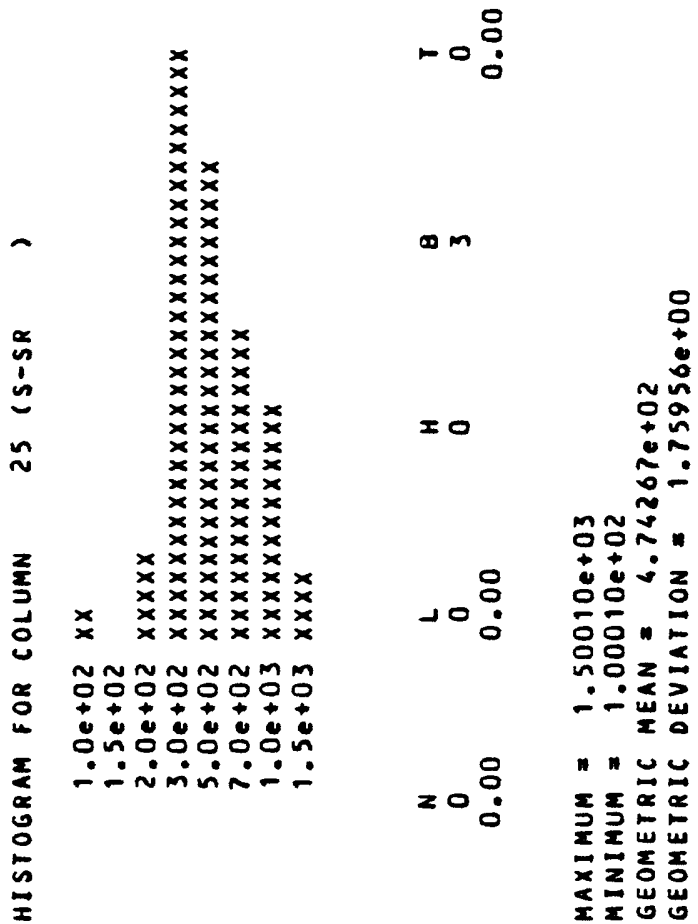
zㅇㅇㅇㅇㅇ음 n nomoa

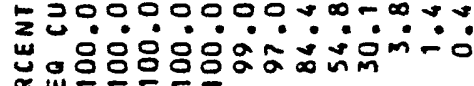
㟧

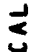

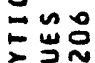

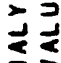

$>$

00 :

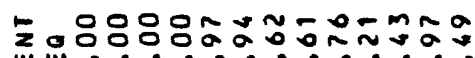

- U山ّó a.

¿

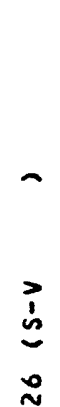

$-0 \stackrel{8}{0}$

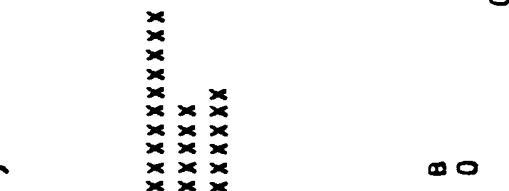

:

$\stackrel{\circ}{\sim}$

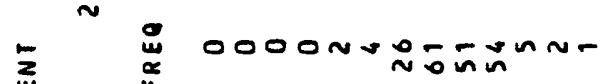
$\frac{2}{2}$

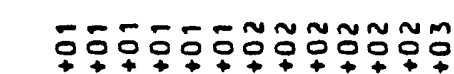

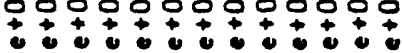
$\sim \infty: \infty: m \sim \infty: \infty: m:$

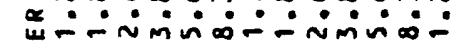

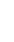

ב! $1,1,1,1,1,1,1,1,1,1,1,0$

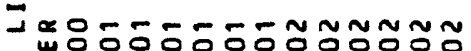

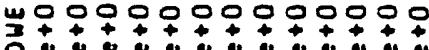

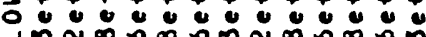

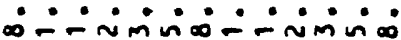

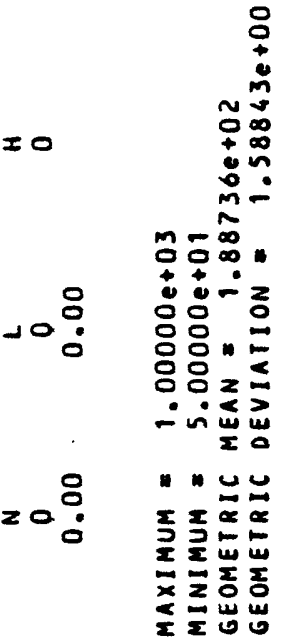




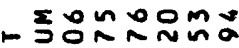

żบำ

s

똥

这

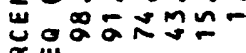

$\underset{\omega}{\infty}$

ט 0 :

- 0 웅

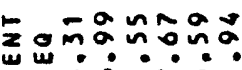

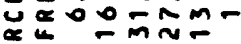

a

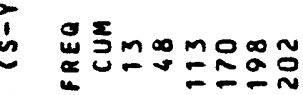

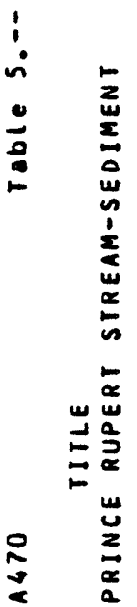

$\stackrel{\infty}{\sim}$

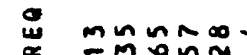

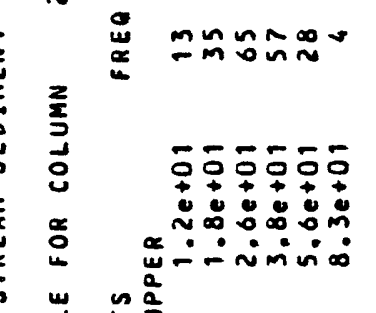

ํํํ

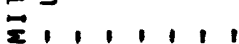

I $\times 8=5=$

느어어여우운

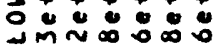

- - - imi

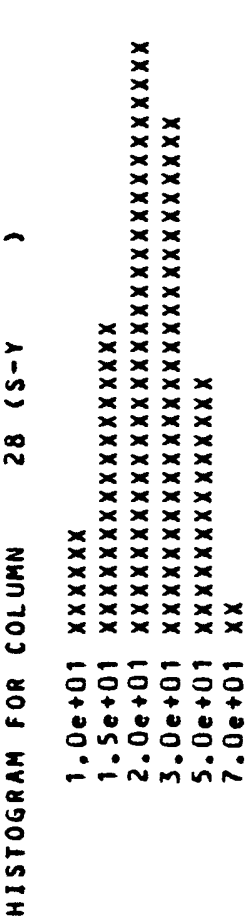

$\infty 0$

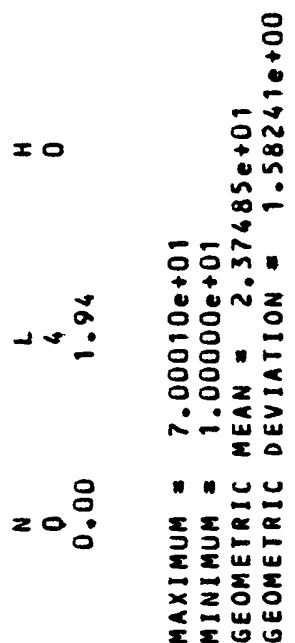


Inaagaoa

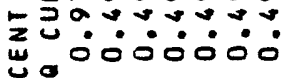

w

แ

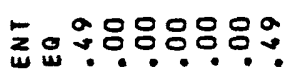

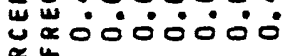
$\underset{a}{a}$

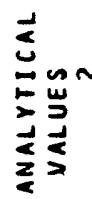

$$
\begin{array}{r}
00: \\
-0: 0
\end{array}
$$

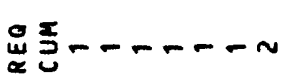

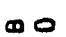<smiles>[Li][Mg]</smiles><smiles>C=CCCCCC</smiles>

ก ก กmmm 웅ㅇㅇㅇㅇㅇ웅 ○ั: ш $\sim \frac{a}{a}$

: - $00000-$

is

i

Io

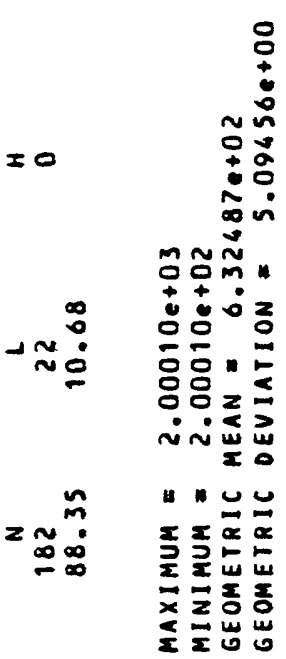



ะ

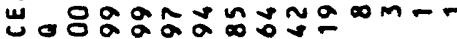
뜽

w

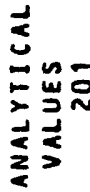

งก:

$-0 \stackrel{0}{0}$

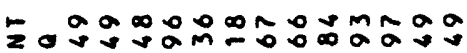

- ứ w

을

!'

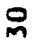

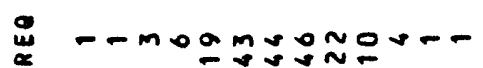

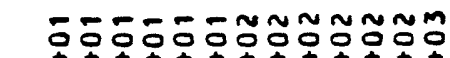

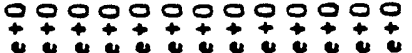
กั

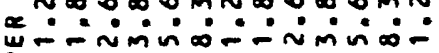

$\simeq \frac{\omega}{2}$

要进, $1,1,1,1,1,1$

$=11111112111111$

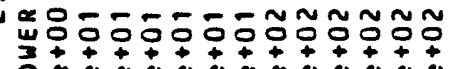

3ㄴ?

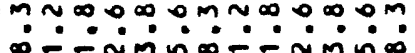

$\infty m$

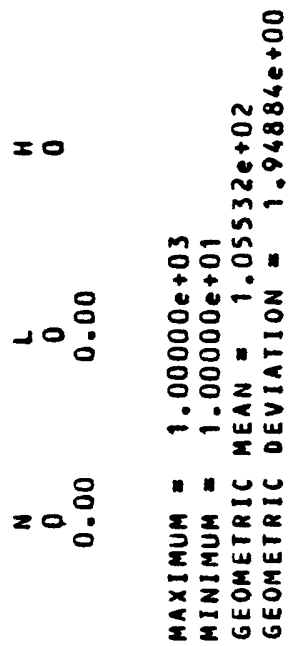


in

- mo은 $=0 n$ zom

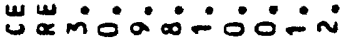

$\stackrel{4}{a}$

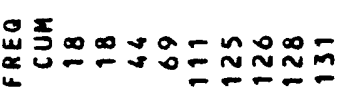
a doonnarnm - Donnatam

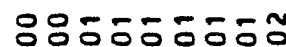

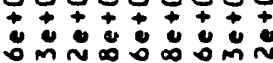

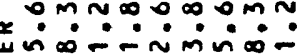
$\sim \stackrel{2}{a}$

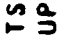

工 1111.111111

숭ㅇㅇㅇㅇㅎㅎㅎㅎㅇ $3 t+t+t+t+t$ ـ mí- - imin

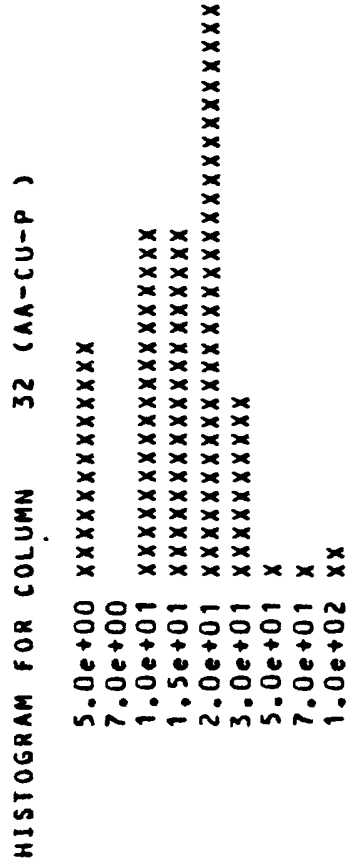

- $0 \stackrel{8}{\circ}$

$\infty$

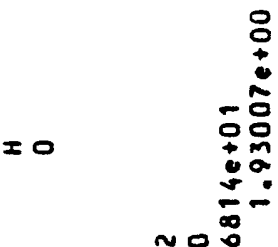

동용.

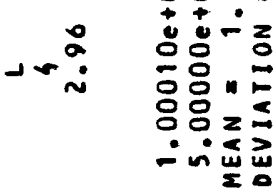

$=0 \stackrel{\circ}{\circ}$

- $\Sigma=0$

골

즐응중

这出出 


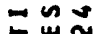

든

丧衣

$-\sum_{\infty}^{n}= \pm \sim \sim$

$\sum_{u}=\ddot{0} \div \div$

แ。大ே的ニ

a

$=$

\section{₹oํㅇㅁํำ}

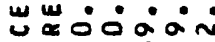

a

!

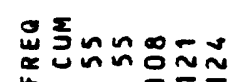
4

i

: ñ ñmm

$\frac{2}{3}$

웅후히 :

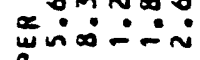
呟

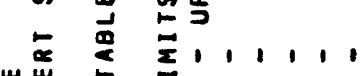

至嵒

4 으음

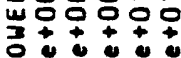

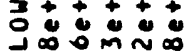
min்-:
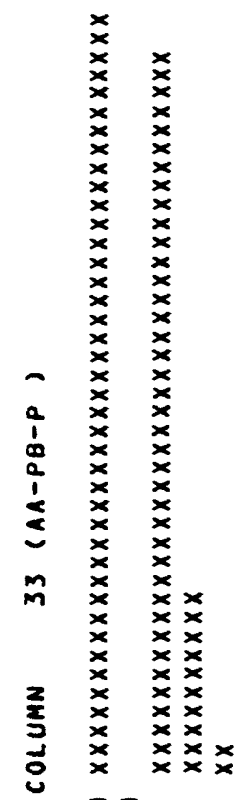

008

-0:

$\infty$

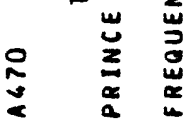

옴ㄷㅁ

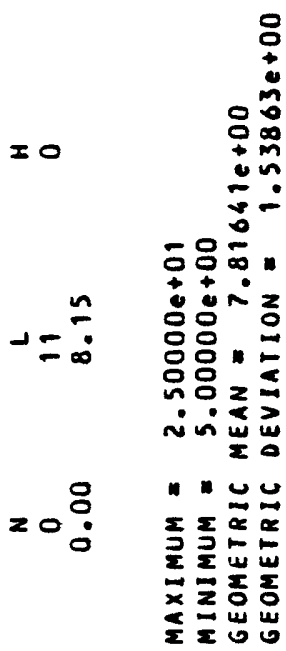




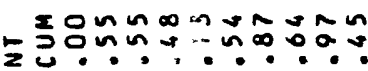

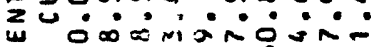

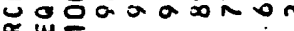

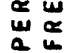

ì

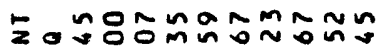
แ 电-0ns=000. 空

a.

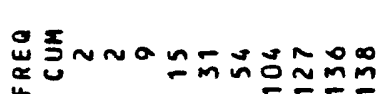

¿ NONOOMONON

을 우웅ㅇㅇ우웅ㅇㅇㅇㅜ 4 $\alpha \because \infty$ แni-nmino-

$\approx \frac{a}{5}$

11111,111

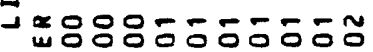
$3+++4++++++$ J in $\underline{\xi}$

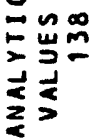

$00:$

- : :

$\infty$
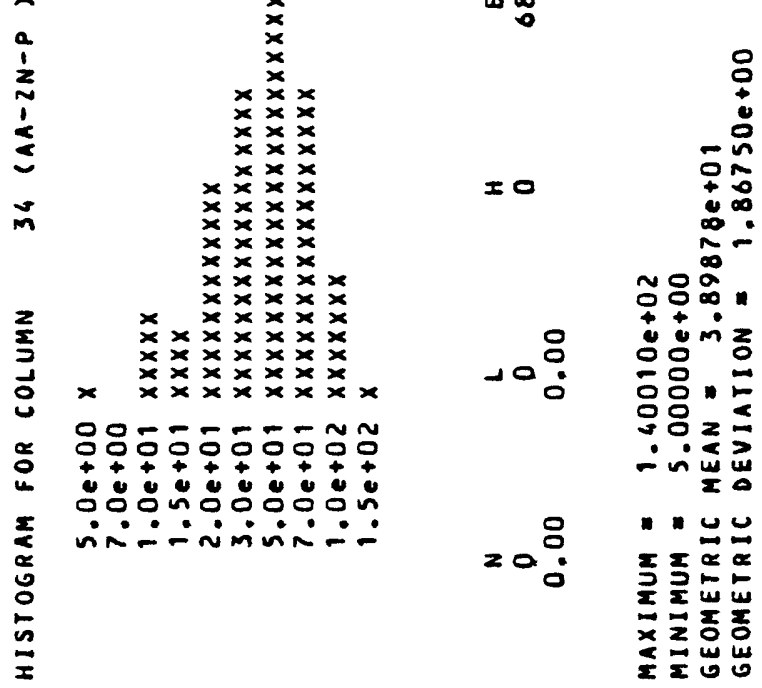
山<总。옹

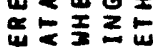

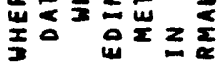

证证出人。

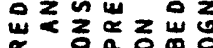

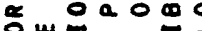

2

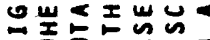

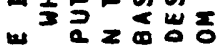

ख

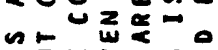

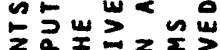

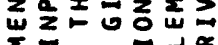

到之山上希

증 -

这全a

J造出地

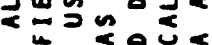

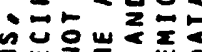

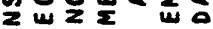

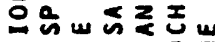

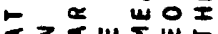

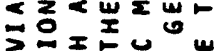

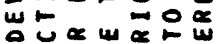

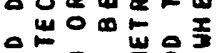

之ी

$<0$ 工 3 인

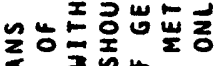

wn

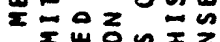

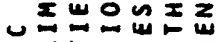

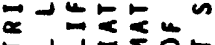

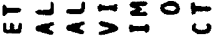

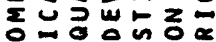

w:n

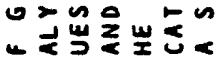

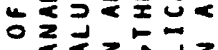

$4 \leq z \leq d z$

崖崖毛。

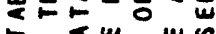

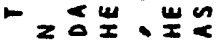

님

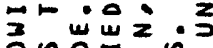

의에

눈

○ـ

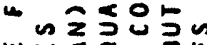

$\omega \rightarrow \leq 0$ का

InI

w

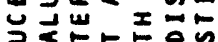

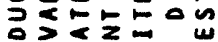

w $=0$

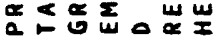

$0<\omega w 0$

(o v uz

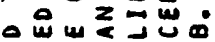

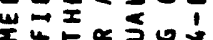

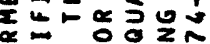

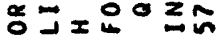

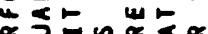

$w$ a $3<w u$

a 200

n $4 \leq k a$

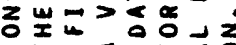

$m b \leq u \leq 0$

䟝

- 000 in $00000000000000000 N 000$

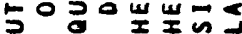

。 


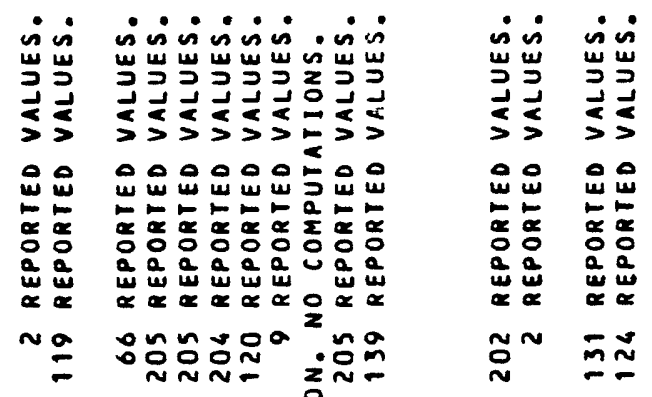

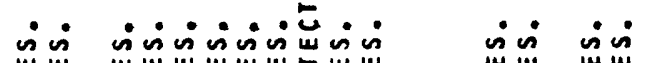

岂岂出岂出出出出岂岂岂岂出

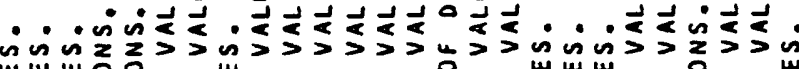

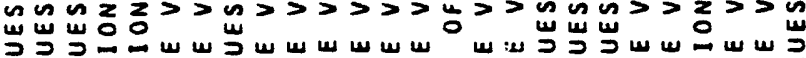

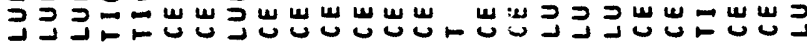

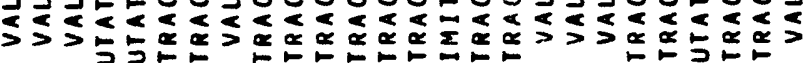

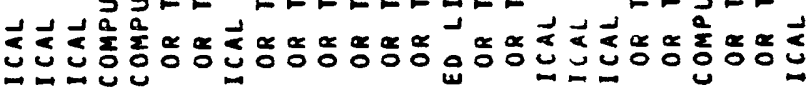

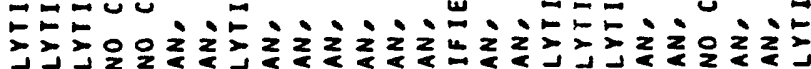

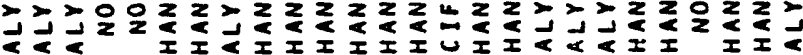

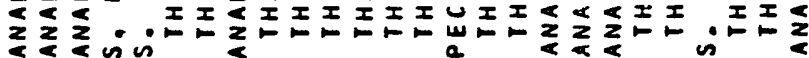

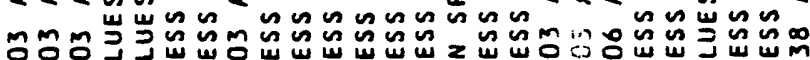

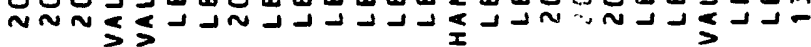

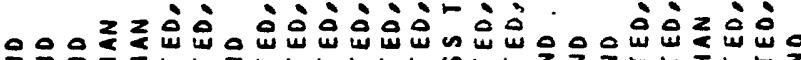

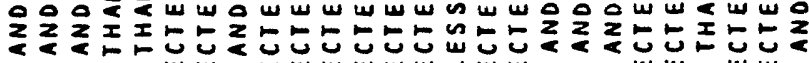

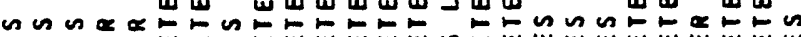

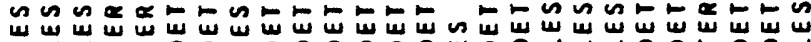

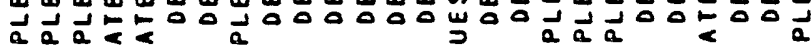

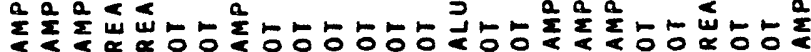

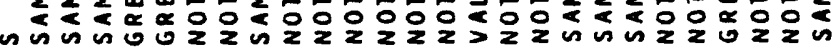

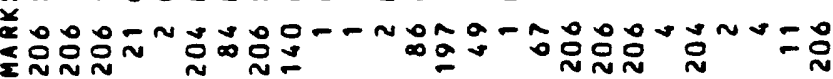
$\underset{\sim}{\omega}$

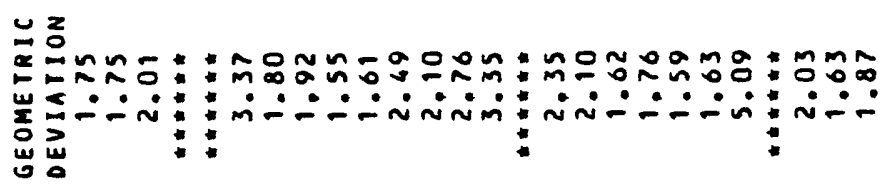

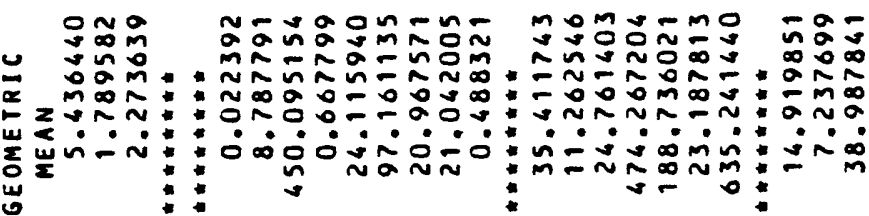

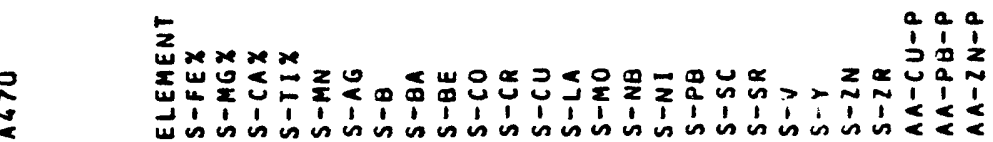




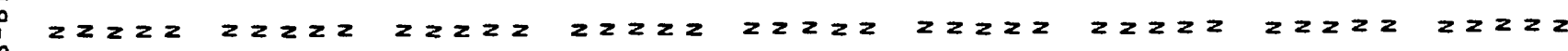

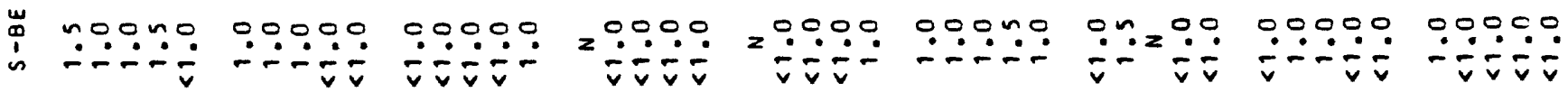

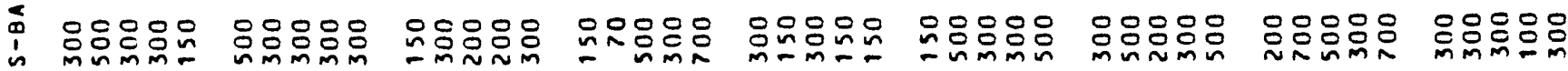

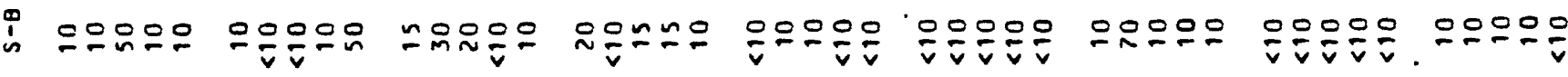

帝

ㄴ?

is

$2 z 22 z \quad 2222 z$

$z \geq z z z$

$z z 2 z z$

$z z z z z$

$z z z z$

$z z z z z$

$z z z z z$

$\because \because 2 z z$

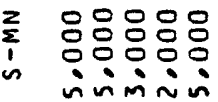

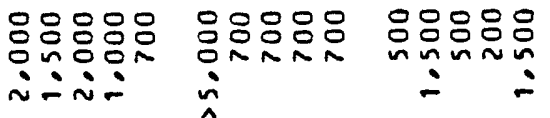

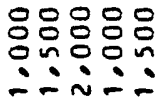

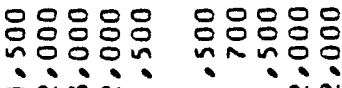

응응은용으

$\because 2 i$ :

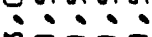

응응응응응

$\because 909$

- in in

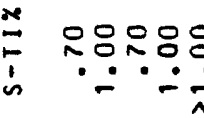

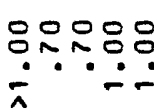

둥ㅇㅇㅇㅜ

00000

00000

0000

00000

00000

- $-\div \div$

$\because \div \div$

$\dot{\wedge}$

$x$
$\vdots$
1
0

imini:

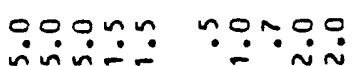

$0.0 \% 0$

$\because \frac{1}{1}$

0000 in

00000

00000

00000

응응읏은

is

ㅇํㅇำ

ㅇํㅇํ․

onomn

$000 \div 0$

$0000 n$

00000

onooo

00000

00000

ש⿱乛龰

0000000000

00000

ooono

00000

00000

00000

nmunm

MNN

¿

ginini

oninni

inm-i

-

ㅇㅇ슴

- míㅇㅇㅇ

00000

00000

ஸिㅇㅇㅇ

ำnㅇํㅇ

$=0 a n$

의

ํㅡㄴㅇㅛ

mojan

nñnñ

ตี山์ํํํำ

ํํํํํํํำ

นํํํำ

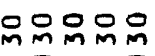

압음옹요

음음음요

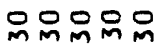

MMMMm

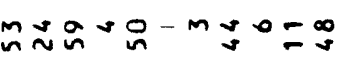

$m \tilde{m}=\tilde{m}$

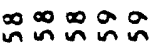

$0 \infty \infty \tilde{n}$

$\sim \sim \sim m \sim$

$\Xi \pm \simeq m a$

$-a \infty \sim \sim$

สฺำ

ทั่งั่ง

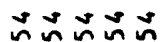

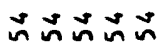

站故的的

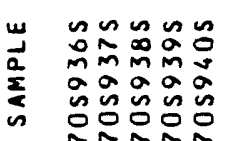

nก⿻上丨丶

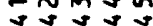

ำกำ nosun

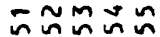
as a $\begin{aligned} & 0 \\ & \text { is }\end{aligned}$ 은옹요
드는
을

운욤의

2.

maja

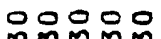

0000

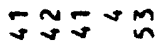

nnoton

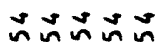

ษ $0 \simeq \approx n$

ำ

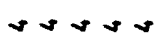

จำ

N NMMM

?am

$m m m \leq m$

$\bar{m}^{n \infty m a}$

OPoos

00000

mNㅡ믄

snmnn

00000

$m M m M m$

$\because \cong ニ=0$

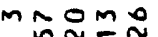

ษัธงท

으는든

mm心

กินกำณ

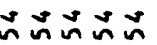

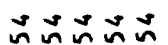

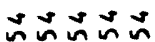

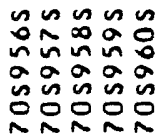

$\because \sim \sim \sim n$ ธํํㅇำ

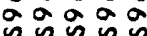

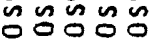

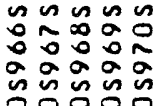

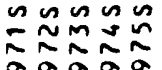

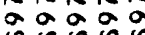
ผูำำ

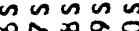

$\sim \pi \infty 2$

a合合

ํํำดัด 


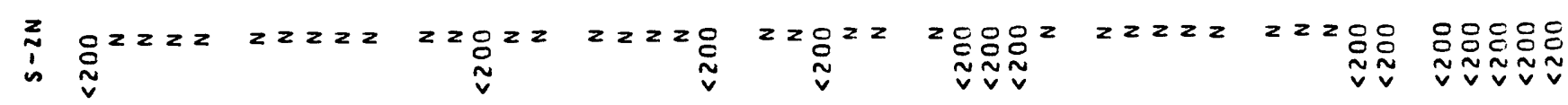

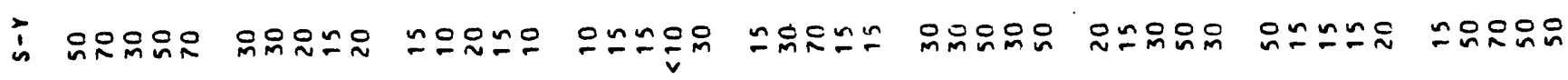

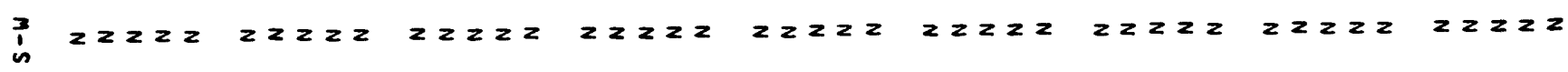

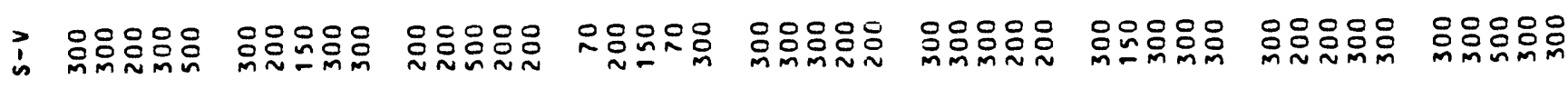

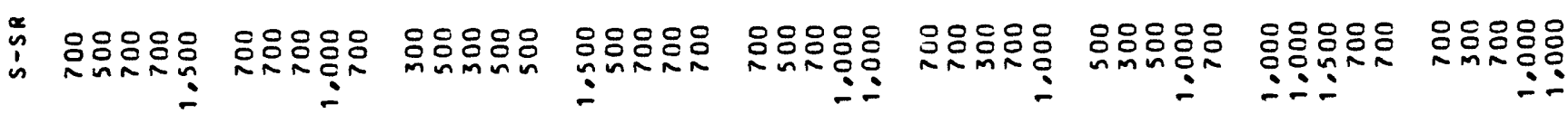
i⿱ 口)

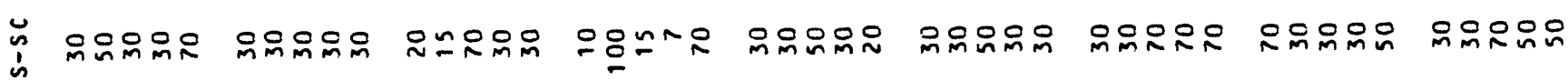
if is

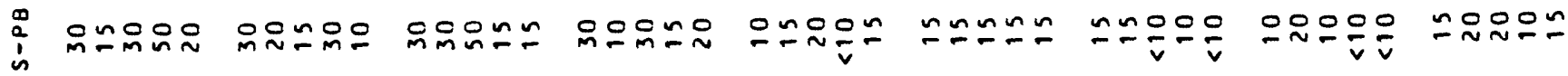

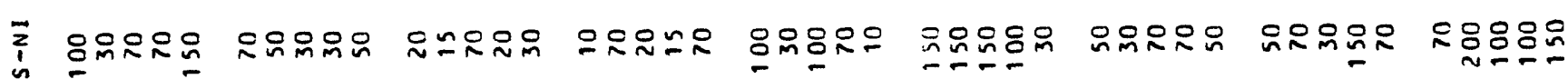

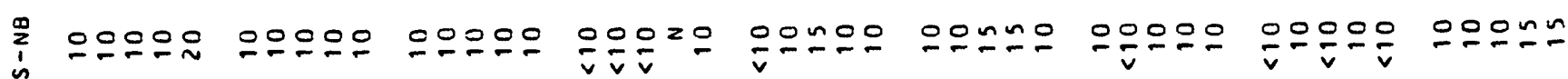

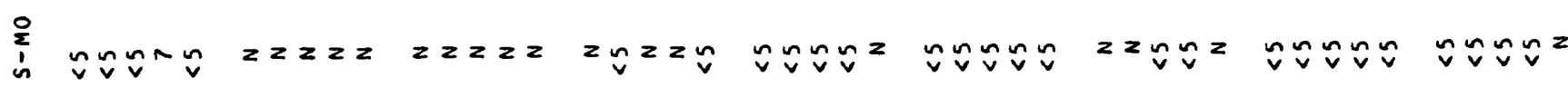

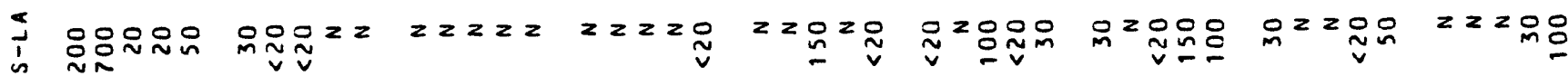

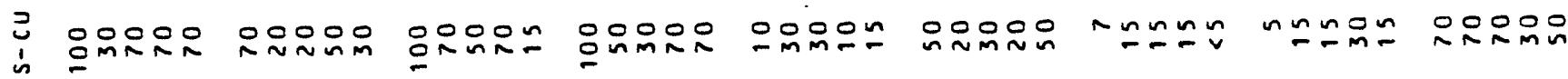

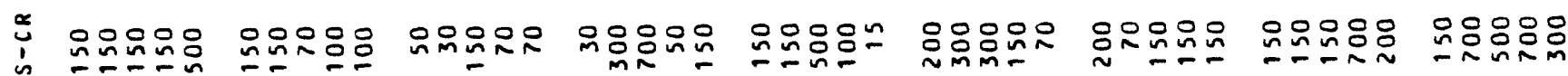

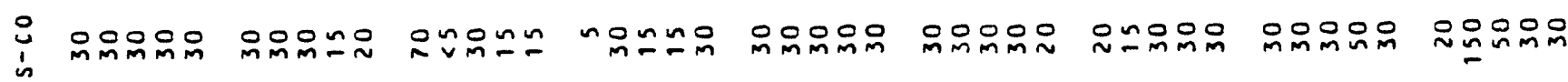

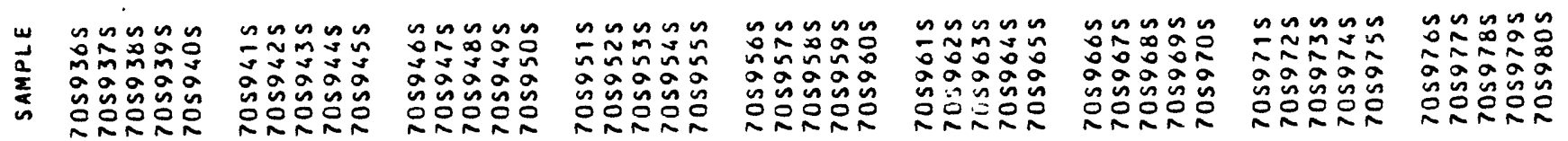




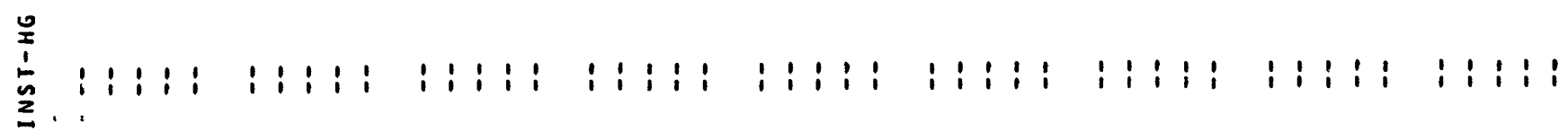

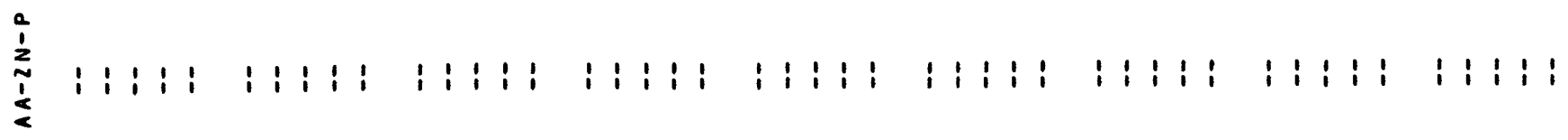
章

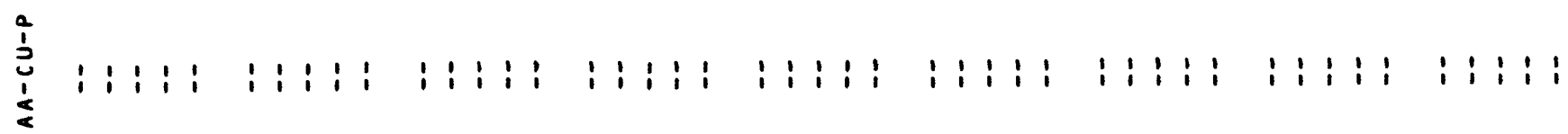

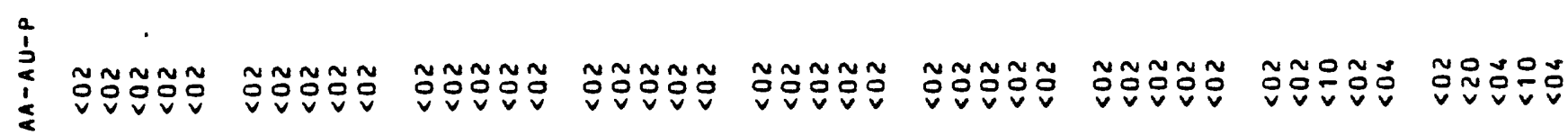

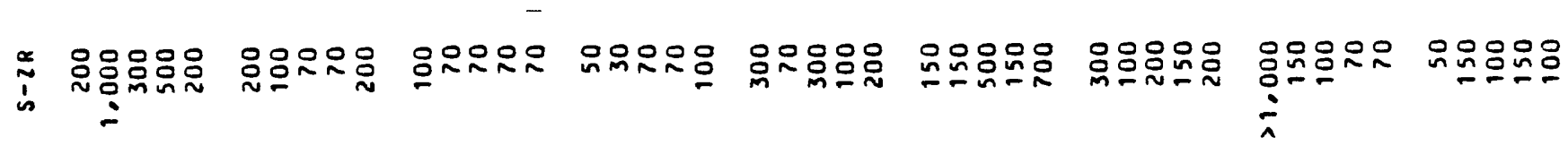

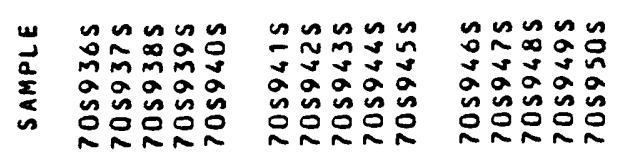

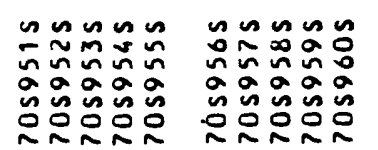

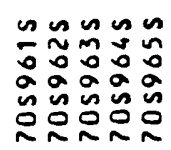

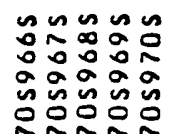

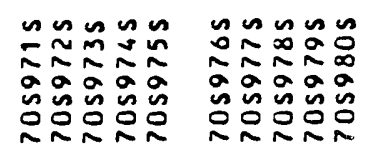




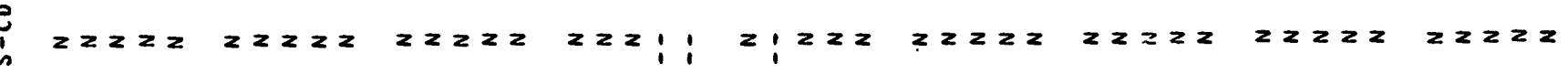

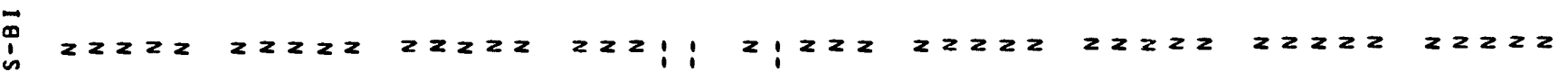

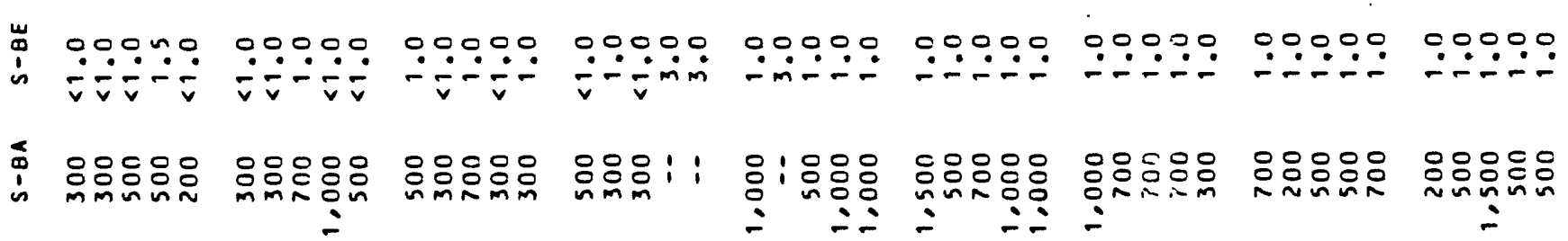

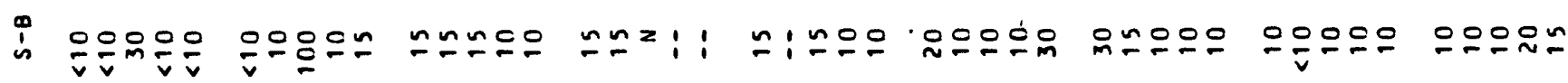

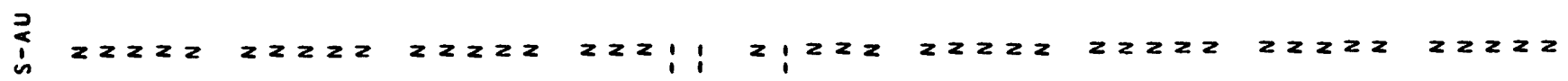

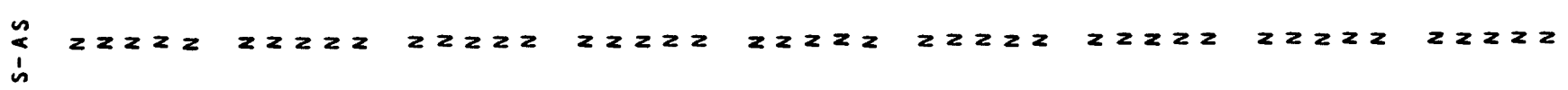

2

용요요

응응응 융영:

的㖣

向市

i : 욤요

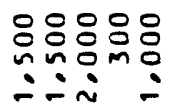

응웅유유

움윰용ㅇㅇㅇ

은등옹응

กับํ:-

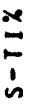

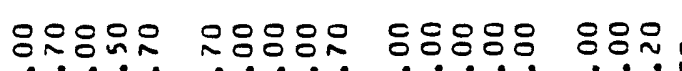

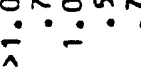

$\ddot{1} \div \div$

$\div \div \div \div-$ $\therefore-$

$\therefore$ í:

$\therefore \therefore$ mi

o:

00000

0 \%.0.

ำ.!!

11000

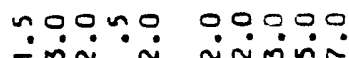

0.000

응용요

앤오운윤요

niñ

oomoo $\sim \sim m m$

产

O००००
जinim

웅․

on 0 :

$000: 1$

$1: 000$

$\because 0000$

- $\dot{m} \dot{i} \dot{0}$

ㅇํㅇํㅇ

00000

जिंmin

nONOO

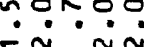
:

웅ㅇㅇ

nㅡㄴ응

00000 - nis

00000 운

0.0 ! : 움

0웅

00000

nónin

00000 승ㅇㅇㅇ

00000 용ㅇ

00000 용ㅇㅇ onmo m $\operatorname{lin} \log _{2}$ - ionan

$\simeq-n \simeq 0$

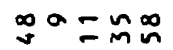

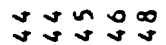

क⿻上丨

ggnnn

n゚ะN요

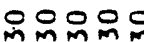

은음음은

잉ㅇㅇㅇ

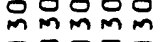

읐있있이

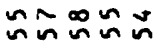

음용ㅇ

minns

ฟ v yao ตnฝññ แnก ññ nี

오은 웅이웅 웅ㅇㅇㅇ

o요요

음잉ㅇㅁ웅

$m m m m m$

$\tilde{n} \sim \tilde{n} \sim$ 을있음음

un $\sim m a$ 00๊ึñ

añnn

$=\tilde{m} \tilde{m}^{N} \tilde{n}$

근

$m \tilde{n} \infty m \sim$

$\checkmark \mathfrak{M}=$

$\stackrel{\sim}{\sim} m \infty$

y요

nைn்

$\pi 0 \infty 00$

mm̃ñ

이의의

은요용

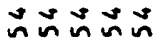

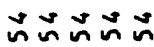

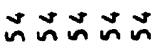

$a \infty \infty \infty$

vosisur

$a \infty \infty \infty \infty$

แั่งป็

แ $\quad n n n \backsim n$ $\bar{\infty} \infty \infty_{\infty}^{\infty} \sim$ a a a a 응오옹

nnn nn

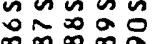
aaa

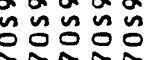

inn

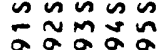
a aaa ํํㅇํํㅇํㅇ
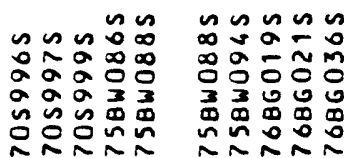

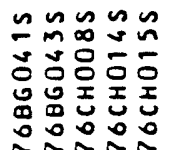

$\sim \sim \sim n n$

$\tilde{\sim} \tilde{\sim} \tilde{N}$

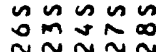

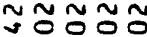

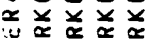

0000 


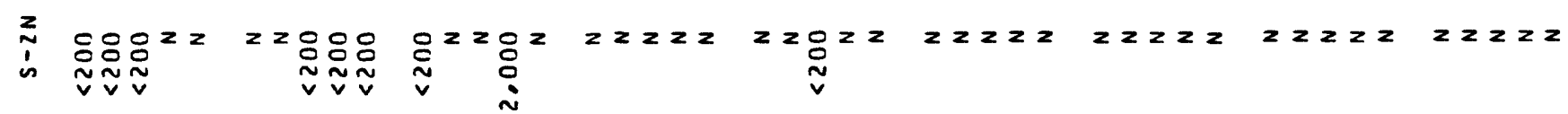

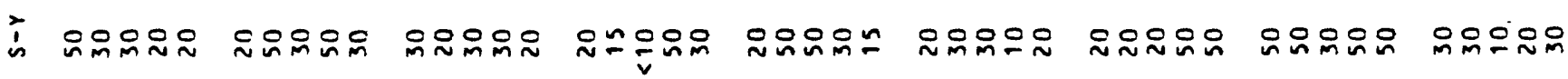

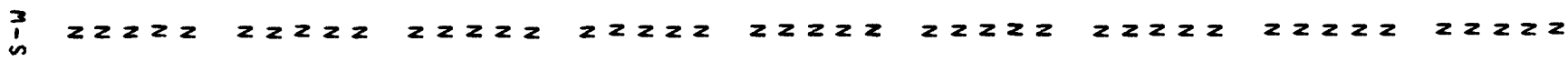

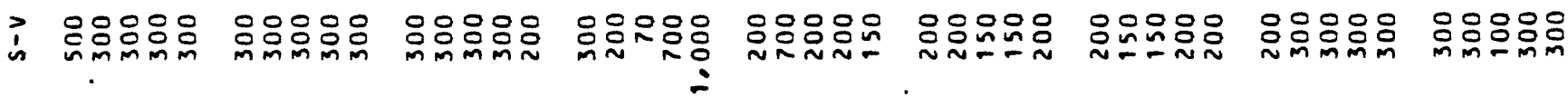

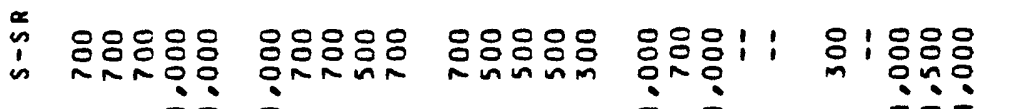

웅ㅇㅇㅇㅇㅇㅇㅇㅇㅇ

옹연온응은

응옹응옹용

웅요

옹옹응응응

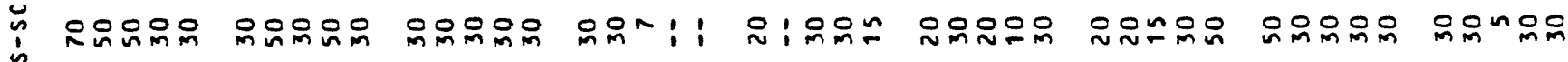
if

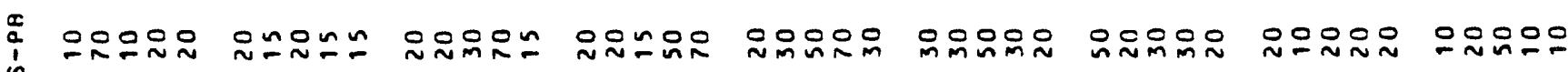

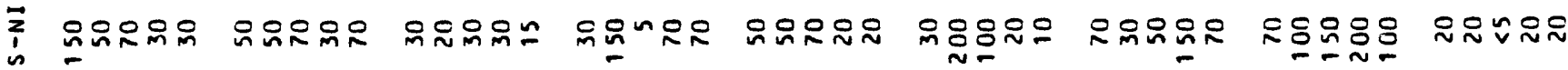

i

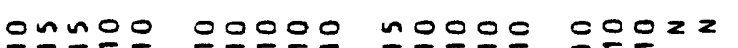

$\sum_{1}^{0}$ ㅇำ $\tilde{v} \tilde{v}$ $z \tilde{v} \sim \tilde{v}$ $\sim \tilde{v} \tilde{v} \tilde{v}$ 웅ㅇㅇㅇ $\stackrel{\sim}{\sim} 20$ ís ì

is 흥은으응 은용요

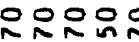
으눈응ㅇㅇㅇㅛ ํํㅇํํ요

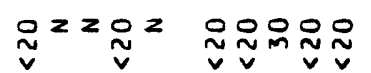
농ㅇㅇㅇㅇㅡ 응응으은 읏요욤 응응응요 용유온요 0000 은요

o000

00000

00000

이요 $n$ mO

00000 N $N$ N

은요입

noogo non

욤요

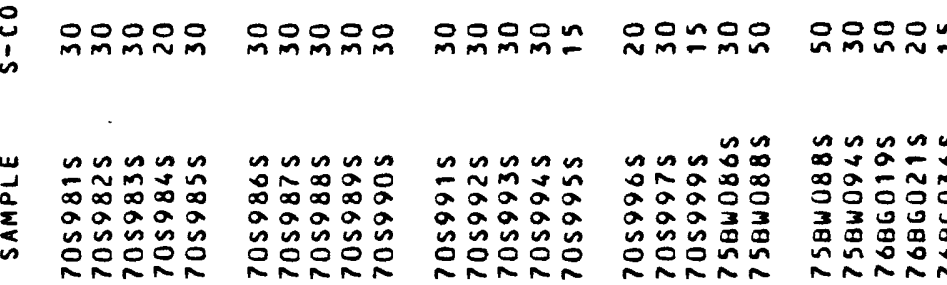

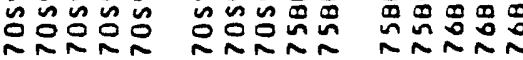

응음오 윰용ㅇㅇㅇ 뜽ํำ

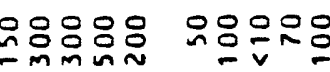

0000n 00000

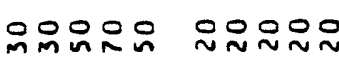

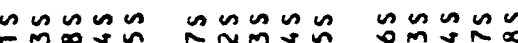

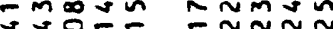
잉ㅇㅇㅇㅇㅇ

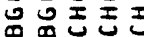

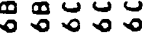
$-\approx \sim \simeq \sim$ I $\approx \approx \approx \sim ⿻ 上 丨$

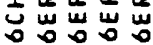

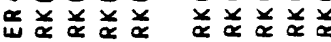
000000000 
号

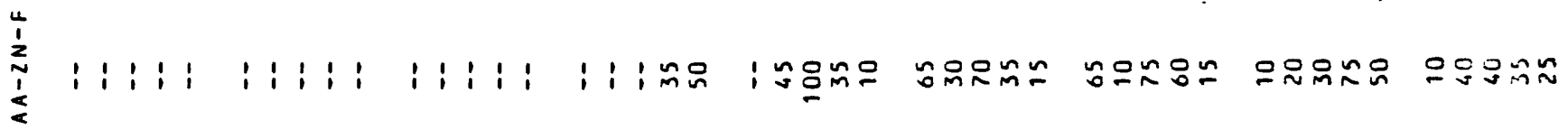

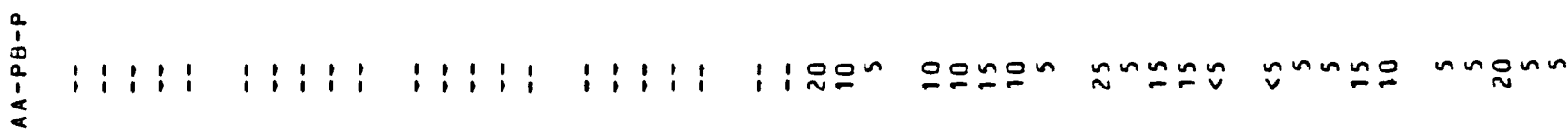

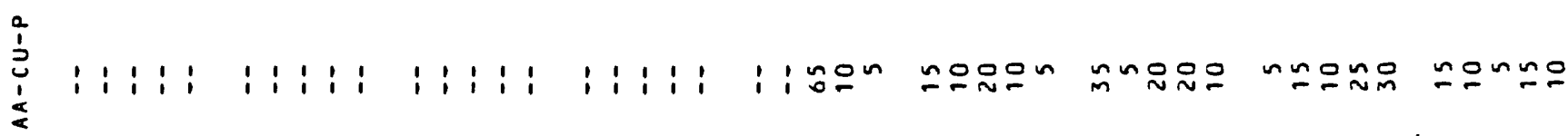

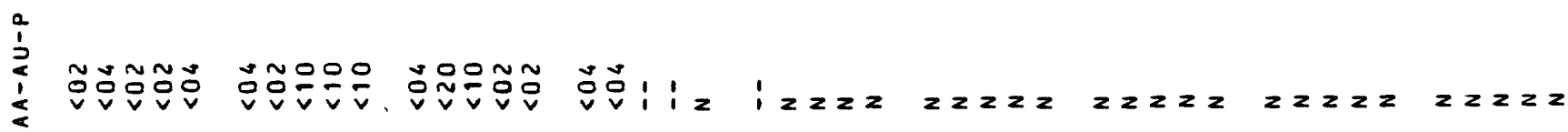

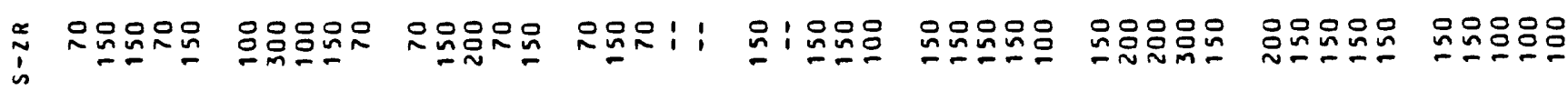

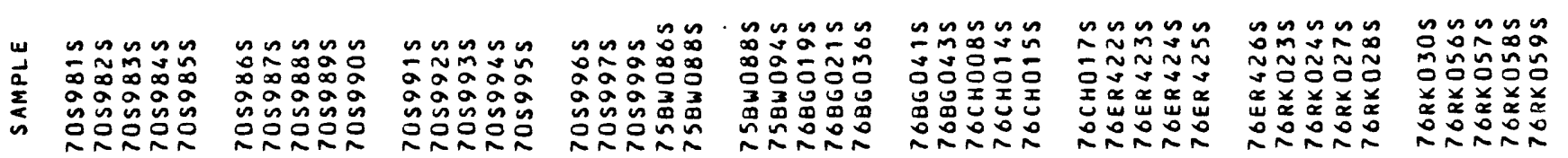




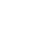

$z \geq 2 z 2$

$2 z 22 z$

$z 2 z z z$

$z \geq z 2 z$

$2 z 22 z$

$z 22 z 2$

\section{0}

운으으음

은은은은은

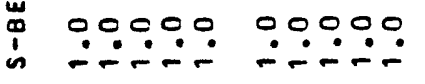

00000

0.000

$\because \because \div \frac{1}{v}$

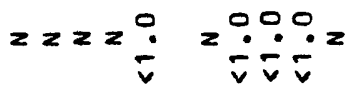

$\frac{0}{\square} \frac{0}{1} \div \frac{0}{2}$

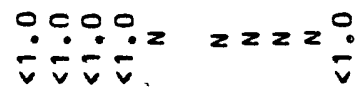

\section{옴웃웃윰}

\section{웅웅응}

0000

\section{更}

옹응응요

$\div$

음웅융

(

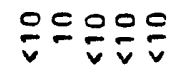

으은은으은

レテテレ

옷윳윰유

웅ㅇㅇ

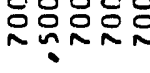

음읏움웅음

은은은은

은은운은은

$\frac{0}{v} \frac{0}{v} \frac{0}{v} \frac{0}{v}$

\section{$2 z 2 z$}

$z z z z$

$z z z z$

i

is

$z z z z=$

$2 z 2 z$

\section{응응웅으}

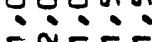

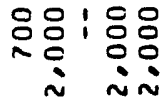

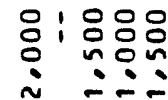

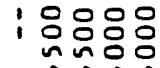

두

옹응융응응

$\therefore \because \div:$

옹웃음응

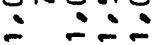

웃응융유

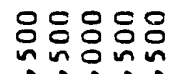

ํำ

응응응음

$\because \because \because-$

온옹응

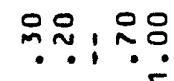

은 웃유운

운응오

웅요웅

웄임웅으

음욱움은

응응응

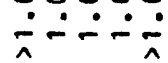

옹용ㅇㅇ $\therefore \therefore$

- $\bullet$.

!.

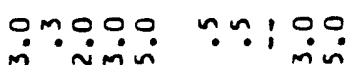

$0: 000$

in 0 .

on:oon

$\because \because 0 \because ?$

ongno

noonn

n-n

- -

-

$0.00 n$ - nñ-

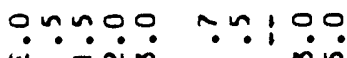

$0: 000$

in: $00 \%$

$\because 90 \div 9$

$\because 0000$

000 \%

$\because \because \because \because$

$\because \because \because 00$

nnm nn $\ddot{-}-\dot{-}-$

\section{0}

00100

01000

10000

00000

onooo

00000 욧 iminimi

iminim

o0000 $n=-\infty$

\section{ニニえぇa}

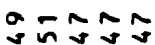

ำำ

䑻舟的的

웅ㅇㅇㅇㅜ

mMㅡ는

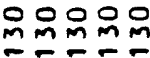

๓ะกำ

=amin

$m \bar{n} \sim \sim n$

이요

战敌敌的

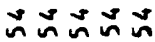

nonun

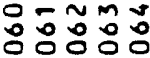

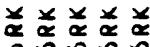

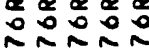

ง $⿻ \backsim$

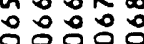

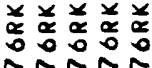

-

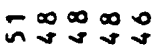

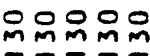

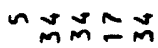

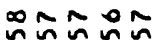

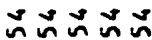

ทำกำ

웅으응

중

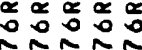

mmำ

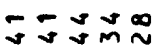
o0000 mํㅡㅁㅗㅡ

น พ

ニュュュ์ ก̃⿱N丶N 00000

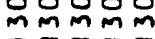

$\sim \approx \Sigma \approx m$

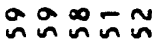

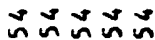

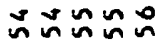

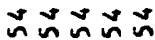

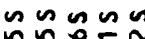
กำำ ×

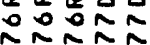

nunus múñô

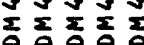

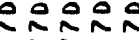
:
บNo⿻上 웅응 mpimpon

กบ 的落敌的

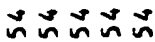

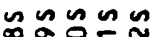

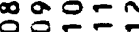
可

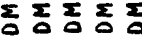

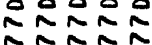
NmnnN in的 $-\infty$ กิก̃̃ 웅요응 M- - -

nீำ

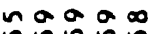

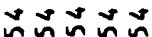

nonas ตะกำ प5 증종조음 ヘลลミล जिimi

00000 invin

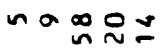
요 우음 웅우웅

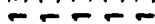

뜸ํํ웅 กิที丶万์

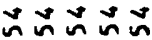

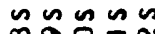
웇 โง

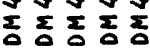

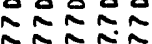

ㅇํㅇำ

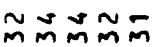

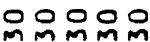
-....

กํำำ munno

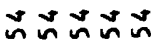

on $⿻$ ns $\sim \sim \sim \sim \tilde{N}$

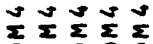

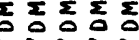
Nㅊㅇ 


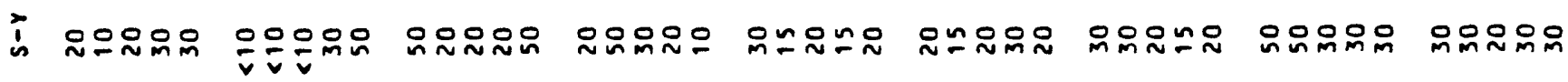
i

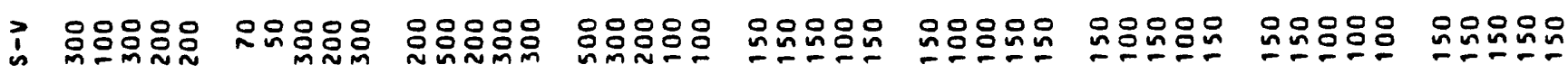

Ц is

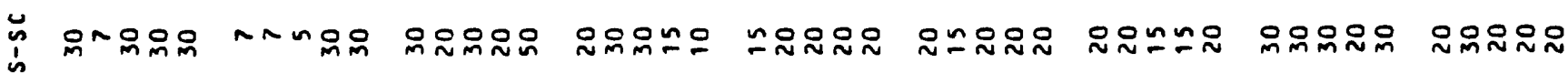
:

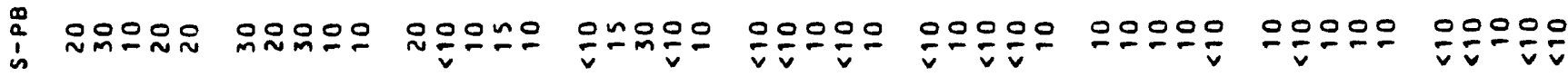

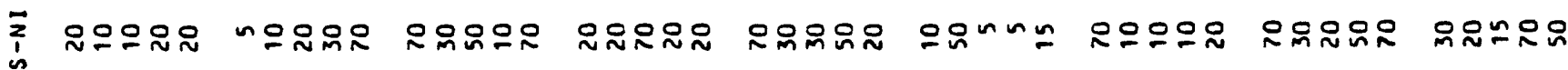

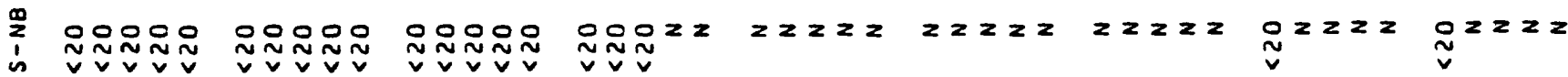

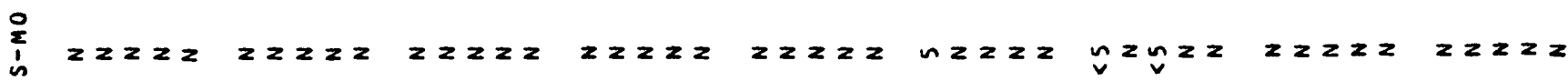

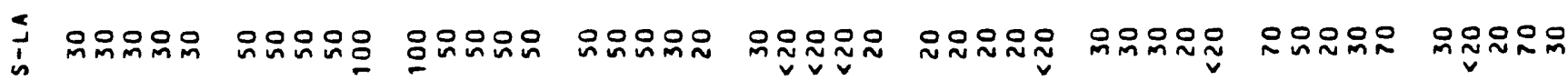

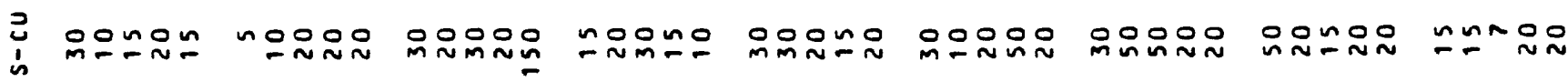

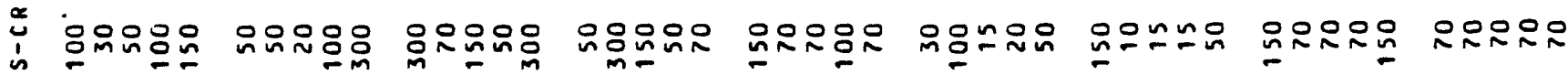

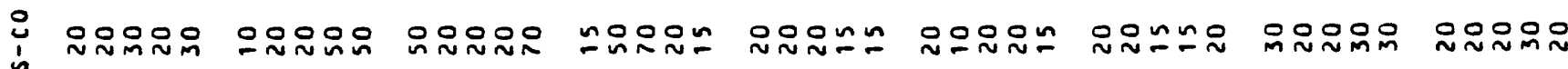

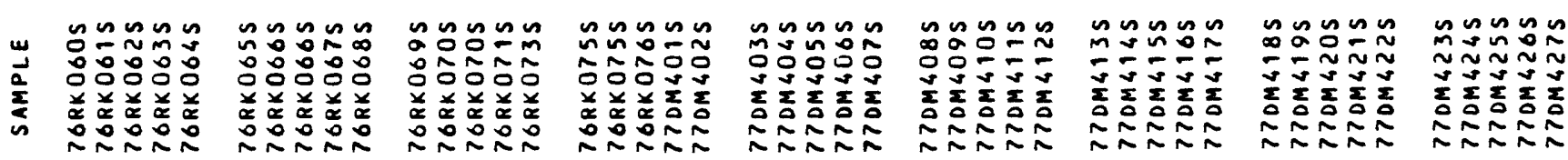




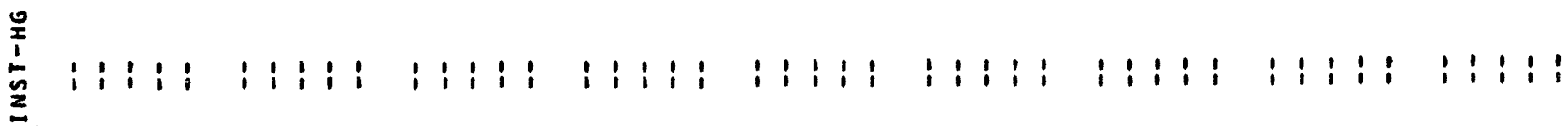

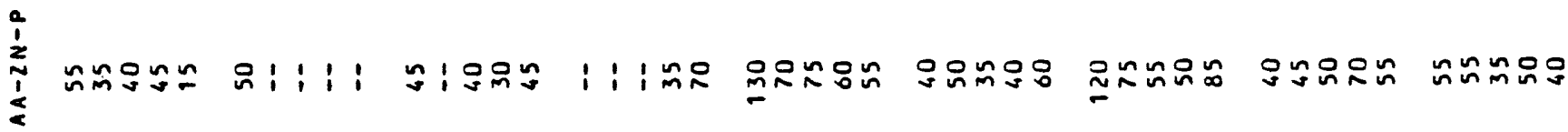
i⿱ 芳

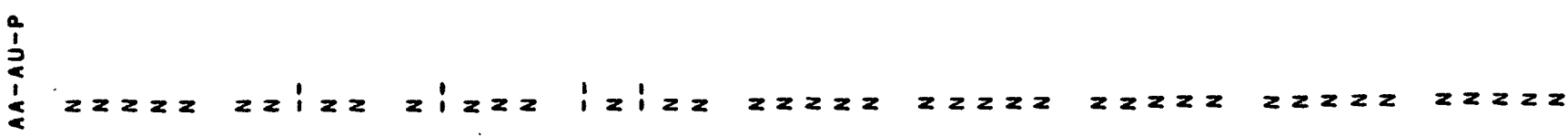

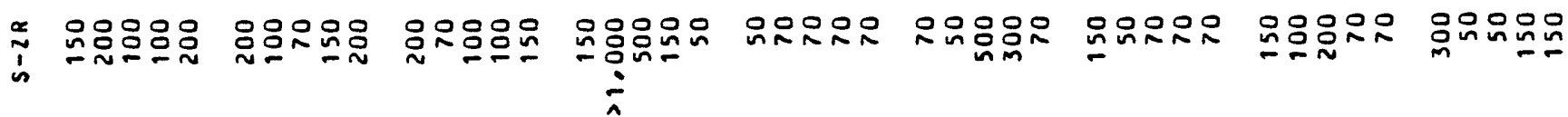

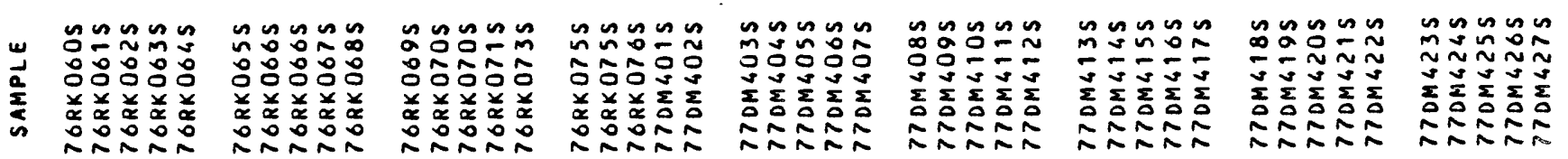


!

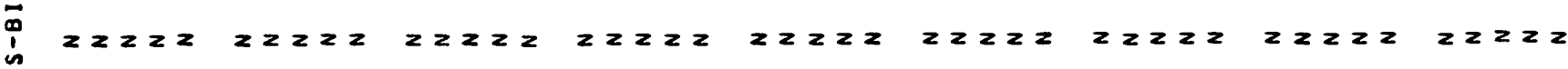

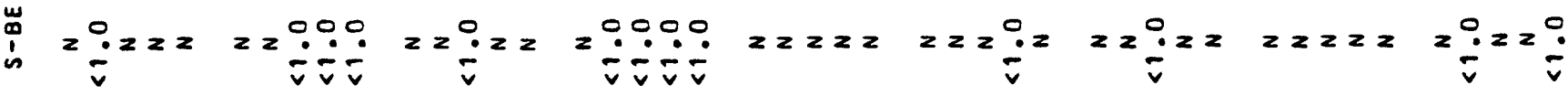

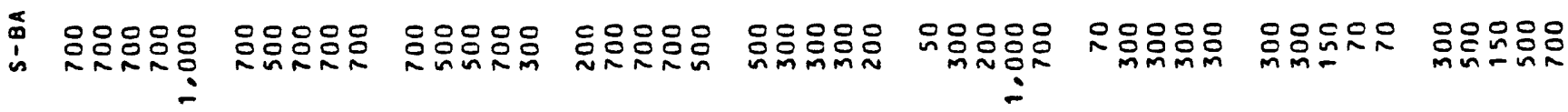

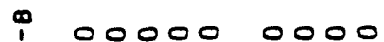

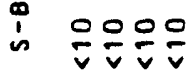

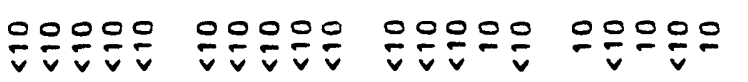

으응으은 으음으으

$\therefore \frac{2}{2} \div$

은은으

is $2 x \geq 2=$

$2 \geq 222$

$2 z 2 \geq$

$z \geq 2 \geq 2$

$2 \geq 22$

$22 \geq 22$

2222

22222

22222

$22222 \quad 2222$

$z \geq 222$

$2 z 222$

2222

2222

22222

2222

22222

i

$2222 z$

22222

2222

2222

$2 z 2 z$

22222

$2 z 222$

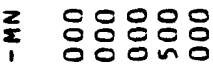

응영응응응

응응응응응

동ㅇㅇㅇㅇㅇㅇㅇㅇㅇㅇ

응응응응응

o요요

응옹ㅇㅇㅇㅇㅇㅇ

$\because \div \div !$

- in :

ㄴ.5:

응응응웅용

$\therefore \therefore-1$

응옹옹ㅇㅇㅇㅇㅇ

$\because \backsim 000$

$\therefore \therefore i \div:-$

응ㅇㅇㅇㅇㅇ

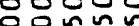

$\therefore \div \div \div$

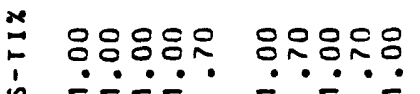

응응응응응

00000

옹ㅇㅇ잉

no00잉

$\div \div \div-\div$

ㄴำ?

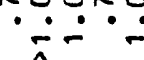

กกกับ

응은응온으

옹용용ㅇㅇ

웅ㅇㅇㅇㅇㅡ

$\because \because \dot{0}$

$\div \div$

i

\subsection{0}

onnunn o0000

nomon

no n n n

o. 0 nn $n$

nnmoo

nn noo

onogo

inimi $\dot{\sim} \dot{\sim} \dot{m} \dot{-}$

$\therefore \div \div \div$

ำำ

$\ddot{-\sim} \dot{\sim}$

$\because$ imin

m-ini

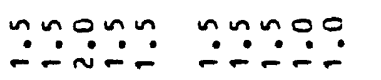

$0 \operatorname{mon} n \operatorname{man}$

ono ? ?

00 n.

$\because \because \because 0 \div$

noำn

กำ. - ini

Onono i i$$
\text { i }
$$

00000

00000

00000

00000

00000

00000

00000 minimi in ininis 웅요

o o on? in $\dot{0}-\dot{0}$

ㅁañ

ํำํำ

$-0-00$

$\stackrel{\sim}{\sim} \stackrel{\infty}{\sim} \sim \mathcal{M}$

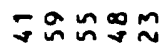

음요 nn

in $v^{2} \sim 0$

mãnn $m \simeq \stackrel{\sim}{\sim}$

nㅡㄴ

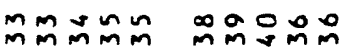

miñ

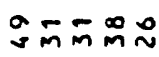

$\tilde{\sim} \sim \sim \sim ⿻$

유

m̄mm

$\bar{m} \bar{m} \bar{m}$

mÉm̃

은음음 음음음

음음음

음음 man

$m \sim m m$ un

$\operatorname{minm}_{m} \mathfrak{m}$

MNㅁㄴ

in $\tilde{m}^{\circ} \geq 0$

กิํํㅇ

$m a \backsim \sim N$

ing $\operatorname{ann}$

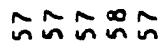

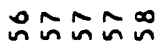

$\operatorname{ñgñm}$

mÉmm

m̃Nå

in in in in

ถูก๊ำตำ

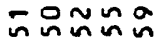

$\operatorname{nin} \sin \pi$

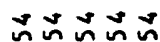

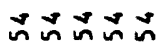

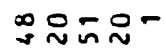

$\bar{m} \bar{m} \bar{m} \bar{m}$

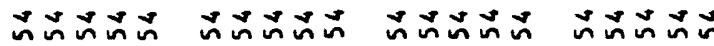

nusosu nosenos

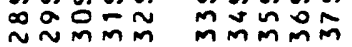

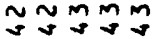

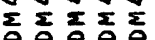

NลNÑ

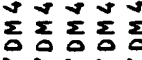

令令

$\sin \sin x$

mán

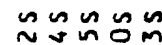

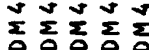

소소

nos 0

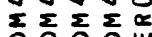

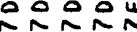

ㅅNㅅN $\sim \sim n \sim n$

$\tilde{\sim} \sim 0 \tilde{n}$

잉ㅇㅇㅇ

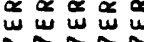

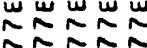

น⿻上丨 Nmmm 웅우웅

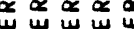
$\hat{N} \tilde{N} \tilde{N}$

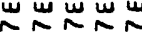

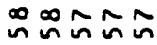

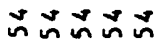

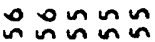

颌战的的

n n n s n mmmñ 잉ㅇㅇㅇ

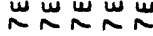
ñ̃̃N
ละกะกต

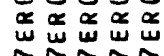
ก̃⿱土龰̃

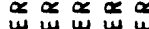

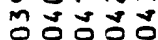

ป⿻n一ำ นทn

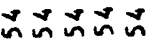

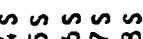

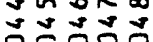

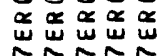
소소 


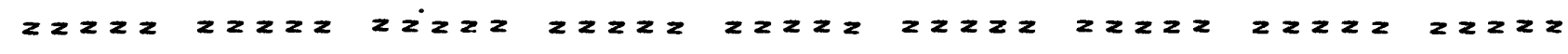

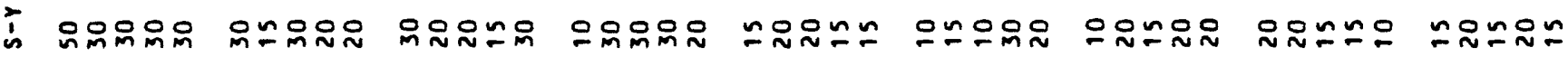

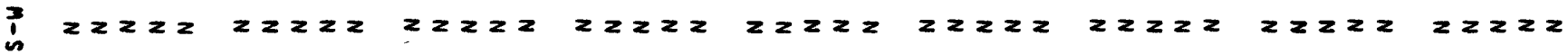

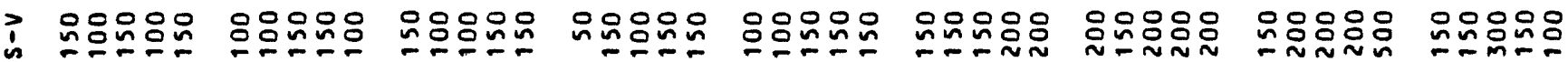
‘

is

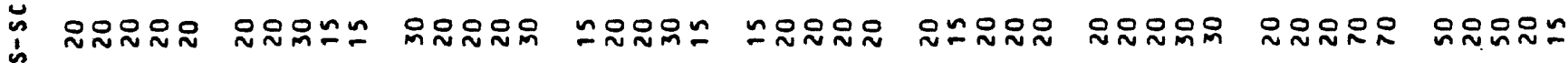
:

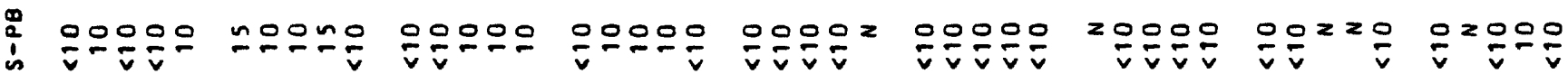

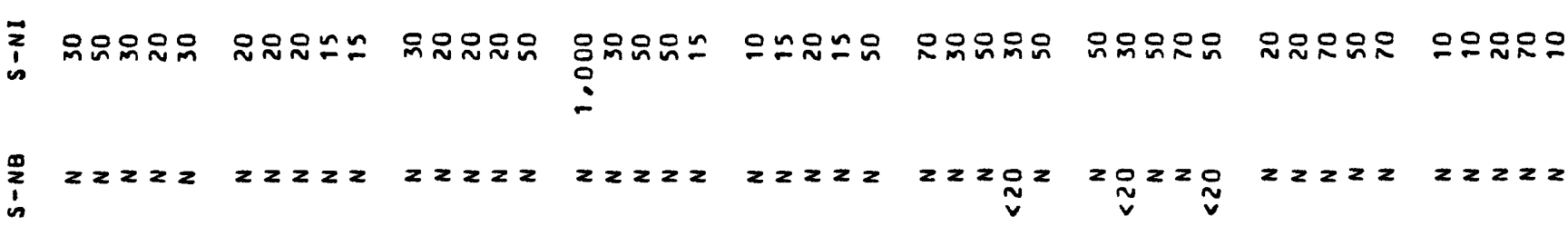

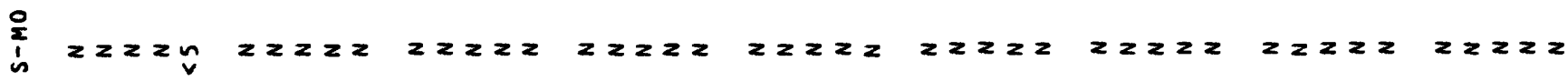
$\dot{0}$

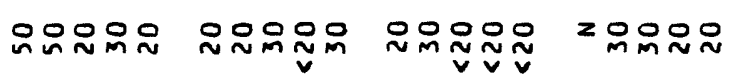

$\underset{\sim}{i} \geq \underset{v}{0}=2$

$z<z \underset{\sim}{i} z$

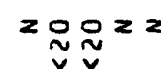

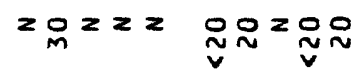

is

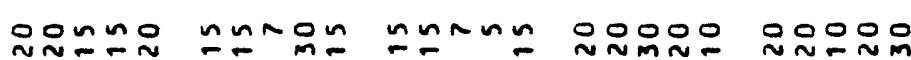

onono

nonoo

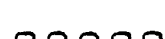

nกะー

is

00000 00000

00000

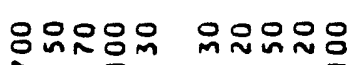

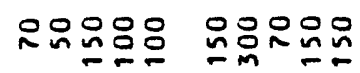

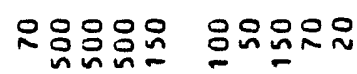

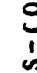

onoon oopinn o0000 0000n

이요요

oo $0 \backsim 0$

onnoo

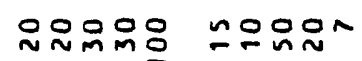

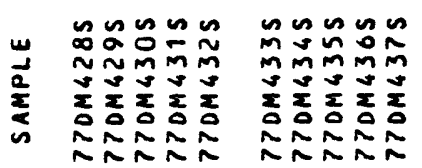

$\cos \cos \theta$

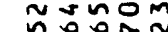

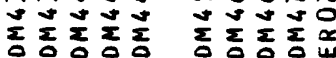

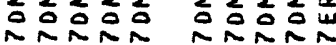

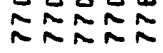

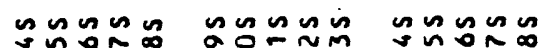
$\tilde{\sim} \sim \mathcal{n} \infty$ ํํㅇํำ

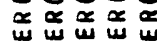

ล

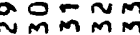

¿̊óñó

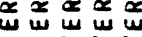



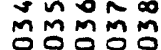

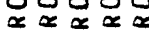

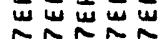

NヘNÑ

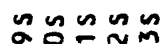

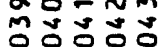

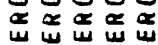

NNN

$\sim \approx n$ ⿰纟勺

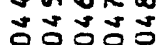

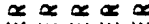
出出令 


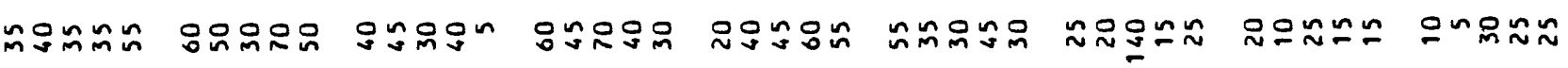

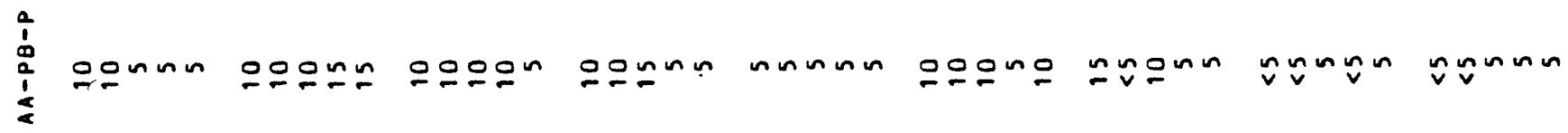
0
$\vdots$
$\vdots$
2

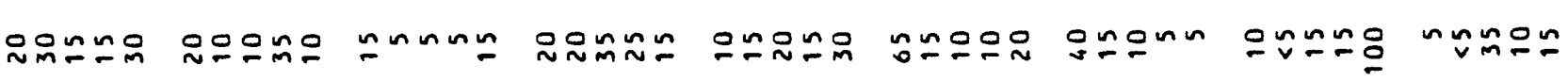
$\frac{1}{2}$ $z z 2 z 2 z 2 z 2$ $z \geq z z$ $z z z 2 z$ $z \geq z \geq z$ $z z z z z$ $z z 2 z$ $2 z 2 z 2 \geq 2 z 2 z$

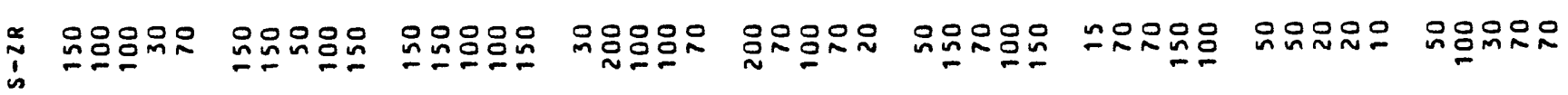

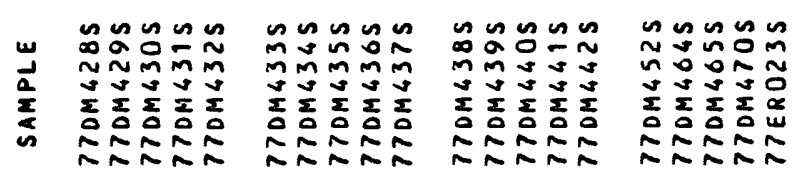
กำก $\sim \sim \sim \sim \sim \sim ⿻ 上 丨$

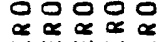
WNNN

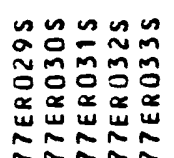

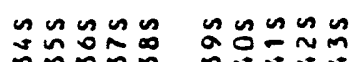

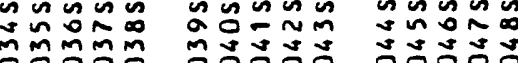

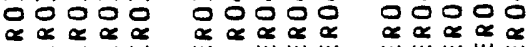
山س山س

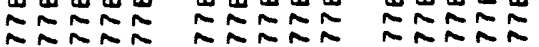


i

is

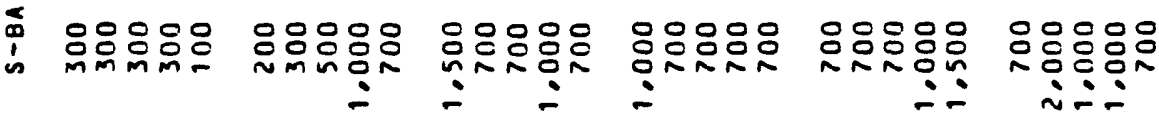

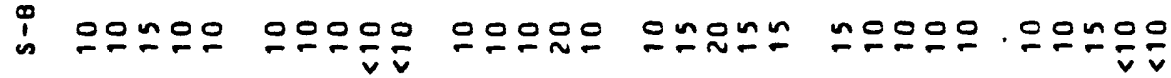

?

n

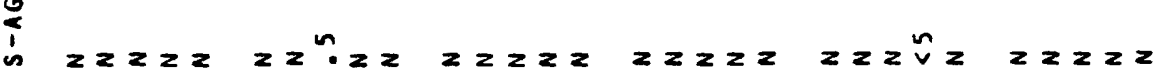

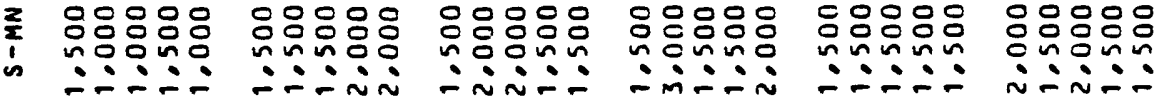

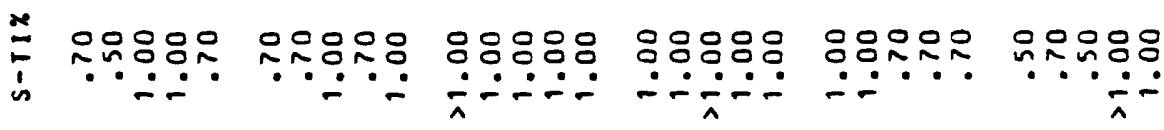

E onnoo 000no no000 nonon nnnoo nno00

i

innogo

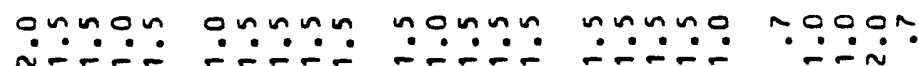

00000000000000000000

0000000000

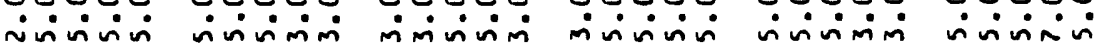

$-m \sim v n$

$0 \sim m \sim n$

$-\operatorname{\sim n} \sim$

$m-0 \infty=$

ป $=0$

ตํํำ

moa 00

$\simeq \approx \circ \backsim \infty$

ñm̃m

$\bar{m} \stackrel{\infty}{\sim} \tilde{N} \stackrel{\sim}{\sim} N \bar{N}$ NO

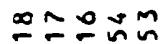

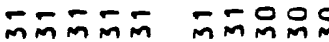

mon

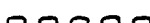

Dzovn

minmm

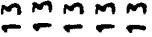

minmm

움음음요

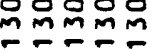

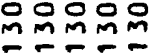

!

ะONM

$\infty m \sim-$

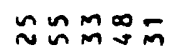

mㄷำ

웜ำ

요 $\sim$

nีnกำmm

in $\infty a$

品的的品

จ的的的

กีบำกํำ

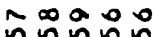

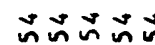

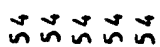

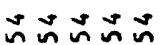

的战战的

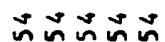

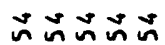

$\operatorname{son} \cos$

in os n is

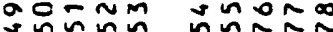

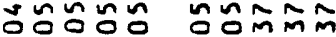

$\alpha \propto \alpha \propto \alpha$

ผN会等

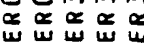

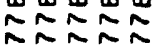

ns nos

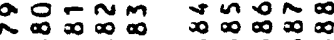

$M m M M M$

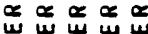

$\hat{\Lambda} \tilde{N} \hat{N}$

in

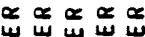

NNNNN

ณnะ⿻上

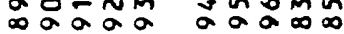

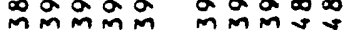

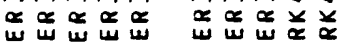

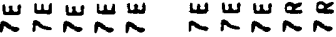




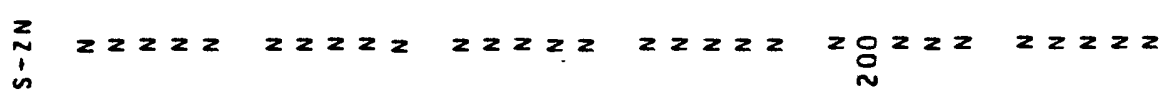

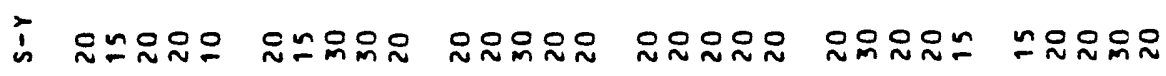

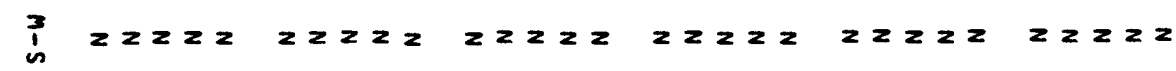

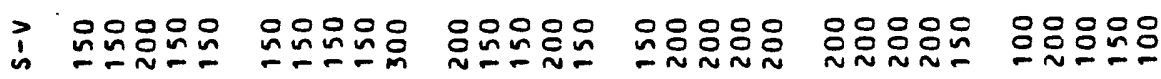

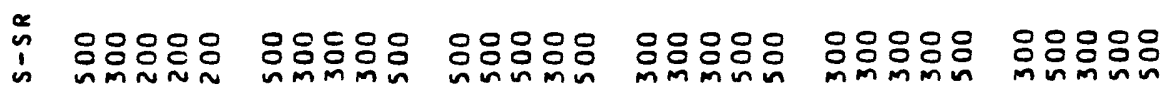

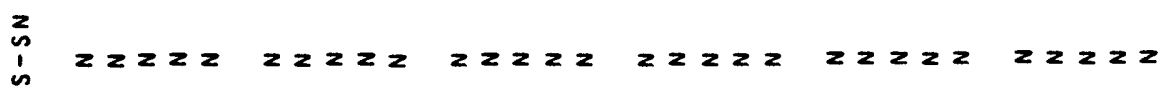

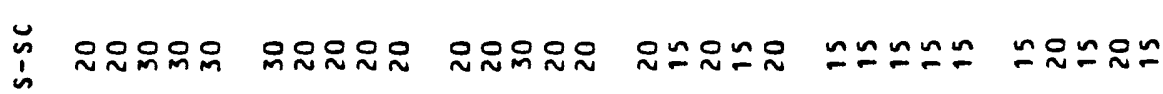

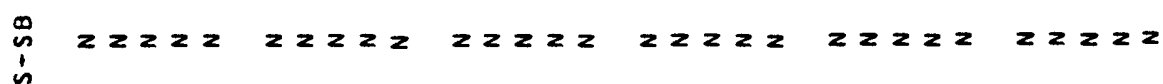

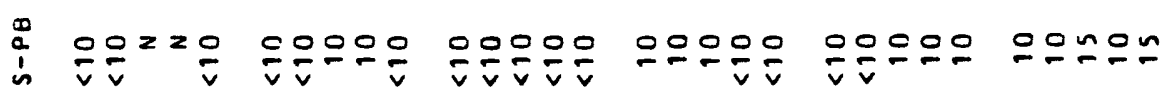

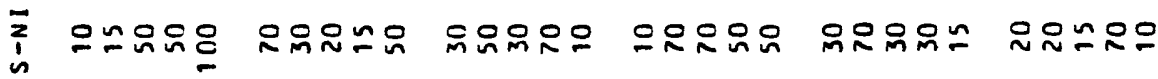

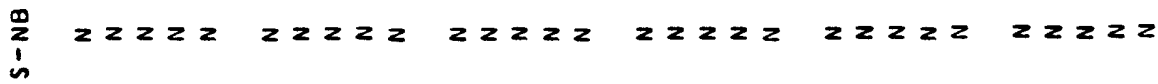

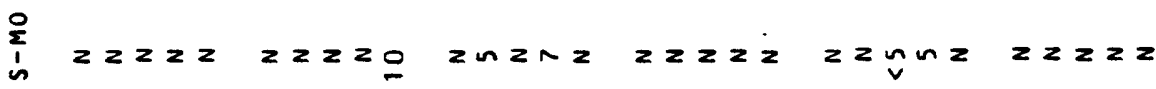

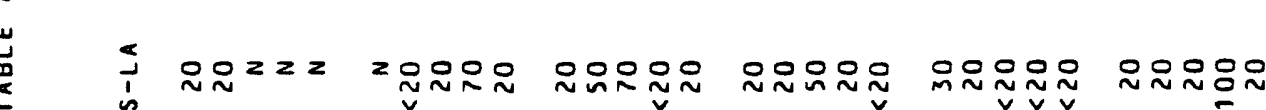

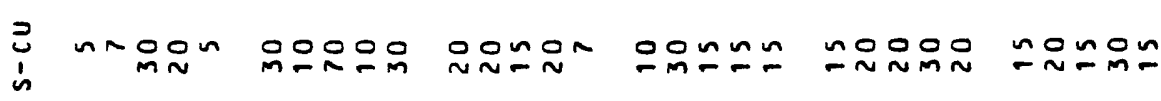

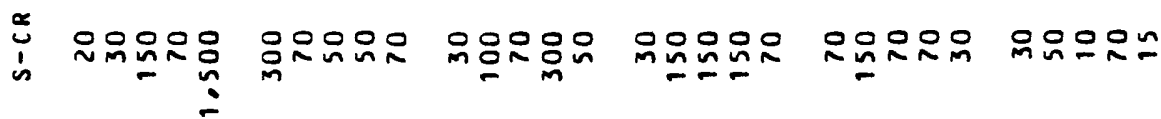

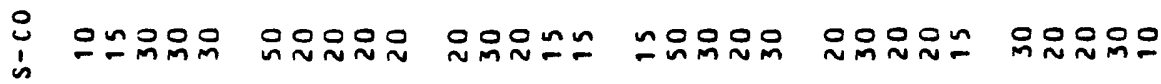

\begin{tabular}{|c|c|c|c|c|}
\hline 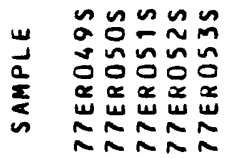 & 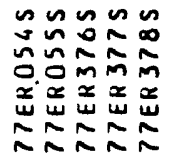 & 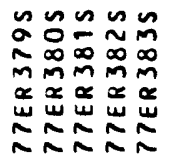 & 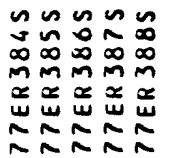 & 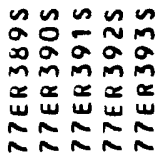 \\
\hline
\end{tabular}




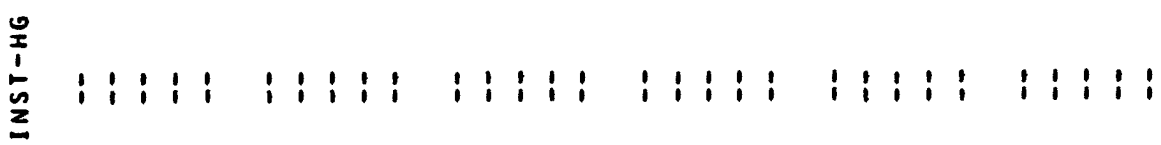
¿ ¿ 方 $\vdots$
$\vdots$
$\vdots$
$\vdots$

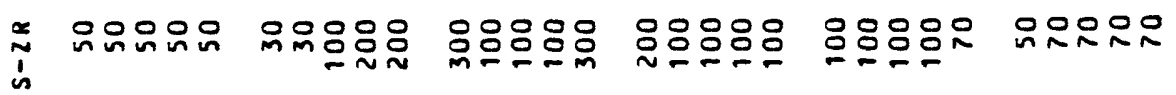

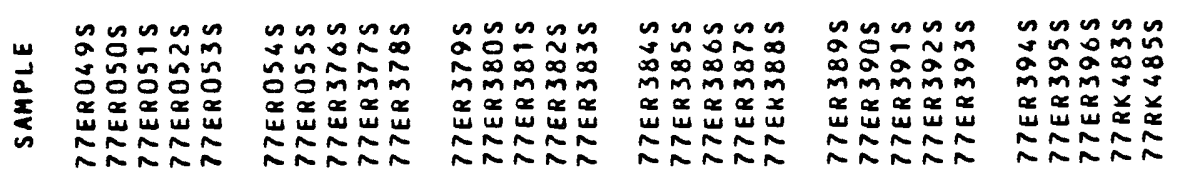




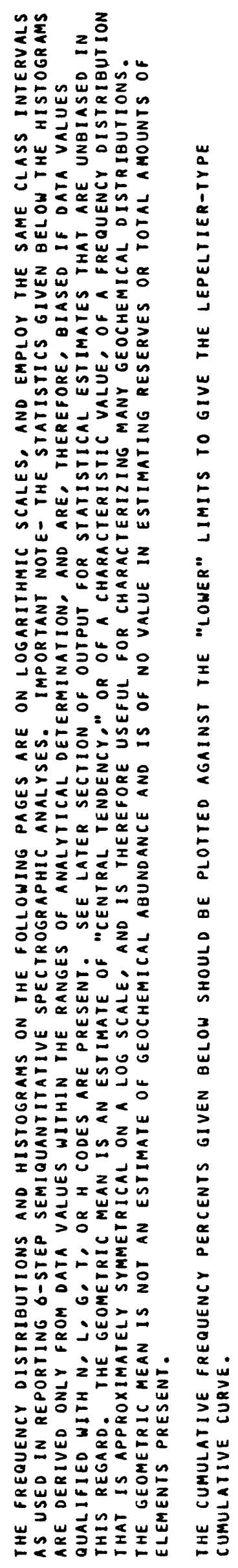




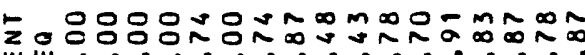

$-0 \stackrel{0}{0}$

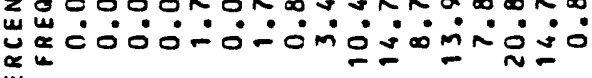
w

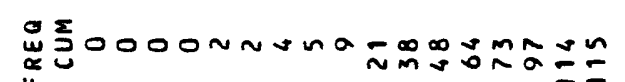

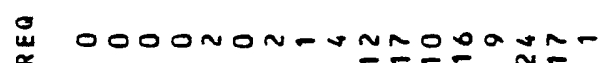
趈

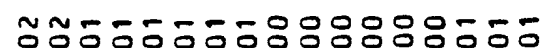

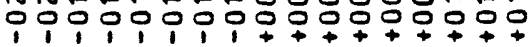

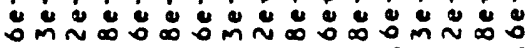

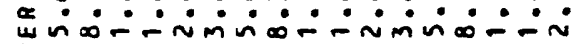
$\sim \frac{0}{2}$ $\vec{\Sigma}_{1}, 1,1,1,1,1,1,1,1,1,1,1$

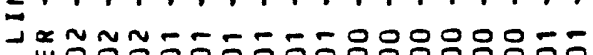

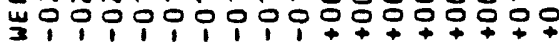

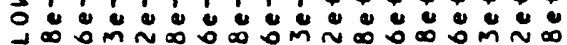

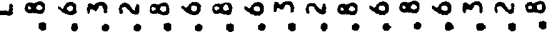

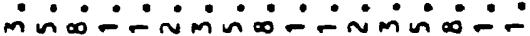

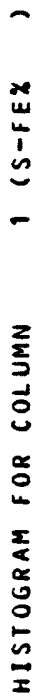

$\Phi \sim$

Io

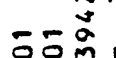
- $0 \stackrel{8}{0}$ t: 두웅ㅇㅇㅇㅇㅇㅇㅇㅇㅇㅇㅇㅎㅇ

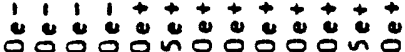

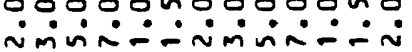




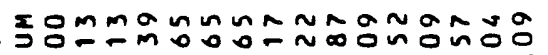
z

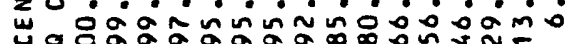

$\underset{a}{\infty} \underset{a}{\infty}$

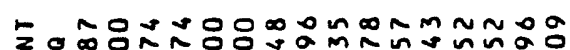
- U⿺辶巛ه a

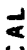

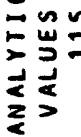
vo: -

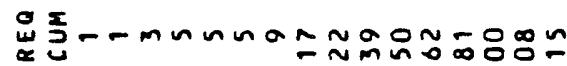

๑n

0
0
$\vdots$
$\vdots$
0

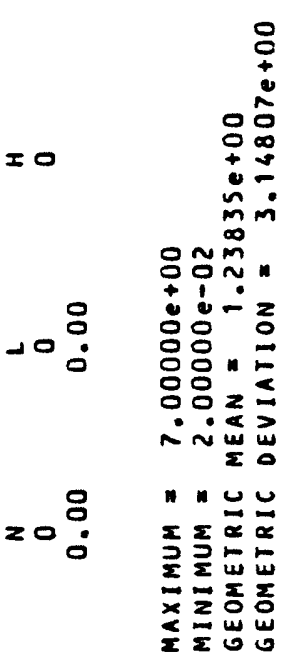

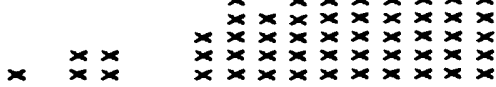

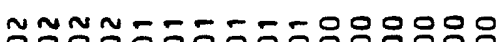

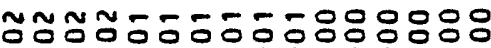
! ! ! ! ! ! ! ! ! ! t+t+t+

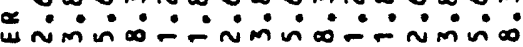
$\sim \frac{a}{2}$

$\Sigma_{1} 1,1,1,1,1,1,1,1,1,1$

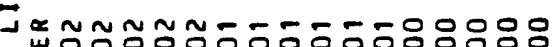

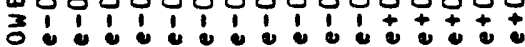

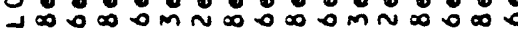

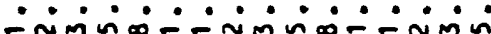

$1111111111+++++4$

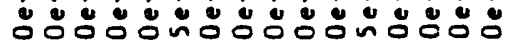
งmin--imiñ-imin

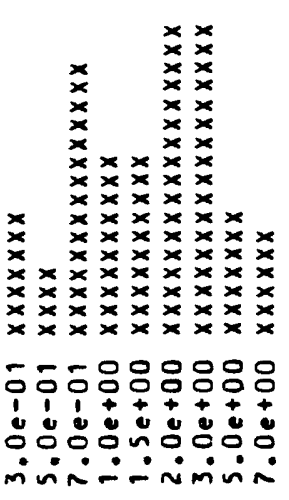

8 
-

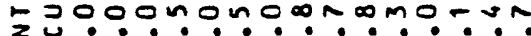
ú

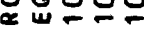
w

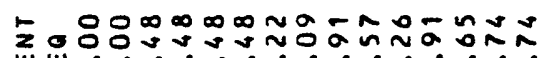

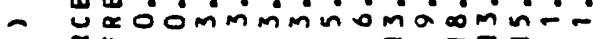
a

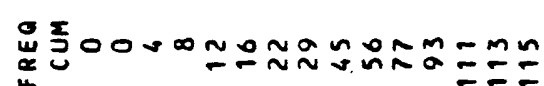

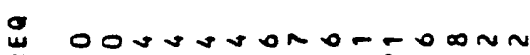
赵

ํํㅇㄷㅎㄷㅎㅎㅎㅇㅇㅇㅇㅇㅇㅇㅇㅇㅇㅁ !

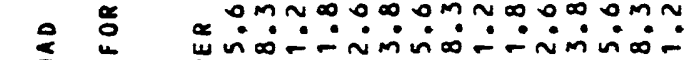
$\cong \stackrel{a}{a}$ 


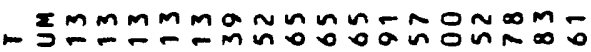
z

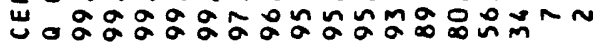
西㟧

w

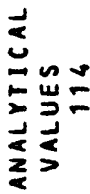

$00:$

-0

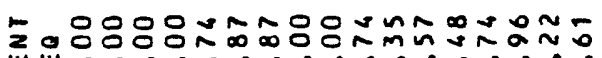

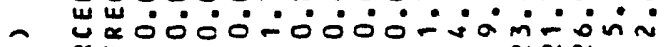
幽 u

운

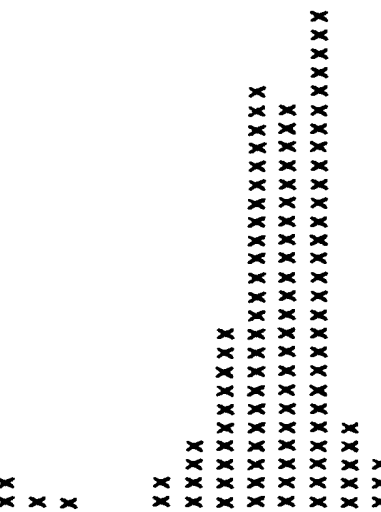
$\stackrel{2}{2}$

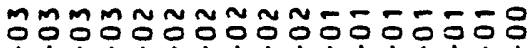

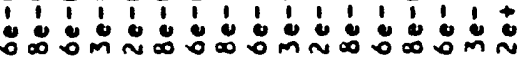

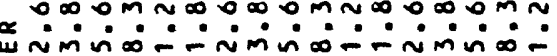
a

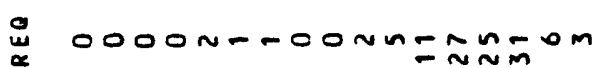

$x \times x$

$x \times x \times x \times x \times x$

E $\vec{z}=1,1,1,1,1,1,1,1,1 \ldots$ ×

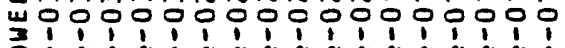

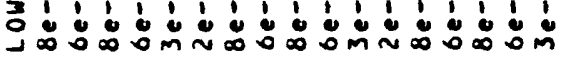

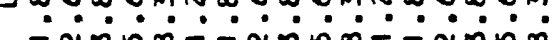

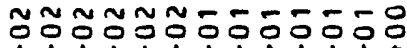

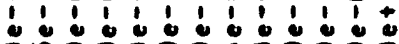

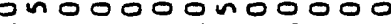

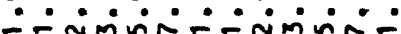

$\infty \sim$

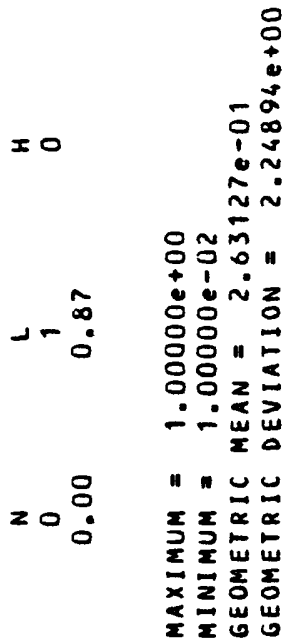


ะ⿻ำ

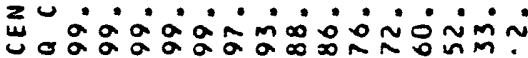
$\stackrel{w}{\infty}$

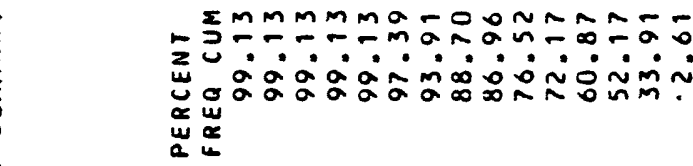

- $0 \stackrel{8}{0}$

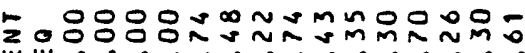

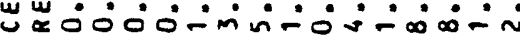

岹

$\sum_{i}^{2}$

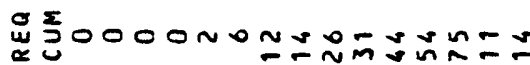
\&

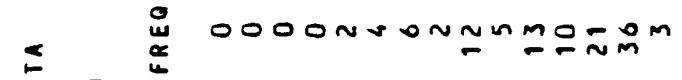

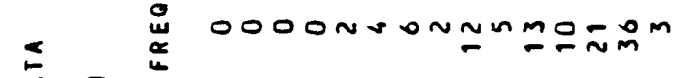

م)

- $\stackrel{8}{\circ}$

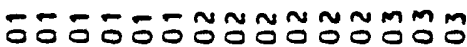

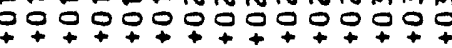
กั

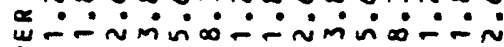
$\cong \frac{a}{a}$

$\infty \sim$

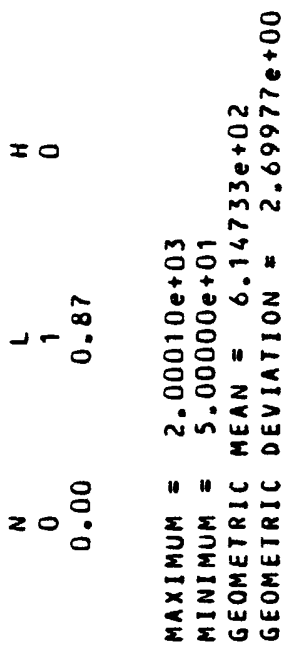




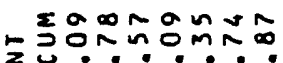

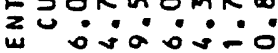
ب요

$\underset{w}{\infty}$

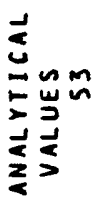

$00 \%$

-0:

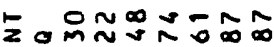
w

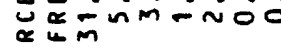

i

$x$
$x$
$x$
$x$
$x$
$x$
$x$
$x$
$x$
$x$
$x$
$x$
$x$
$x$
$x$
$x$
$x$
$x$
$x$
$x$
$x$
$x$
$x$
$x$

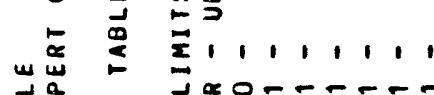

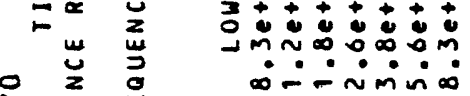

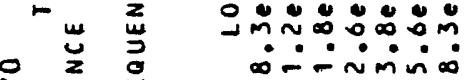

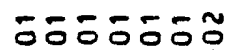
$+7+t+t$

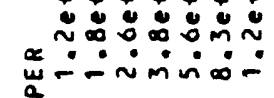
$\sim \stackrel{a}{a}$

11111111
$=00=-5-5$

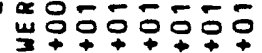

i oーsกm-

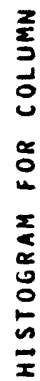

$\infty \sim$

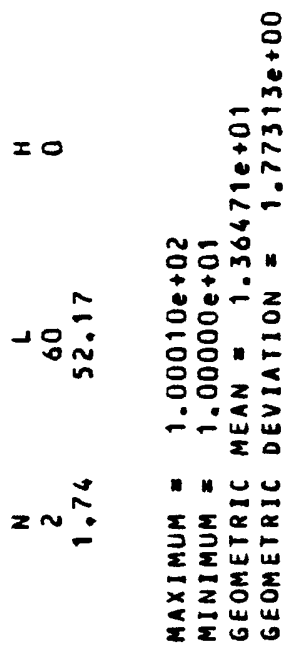




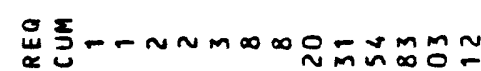

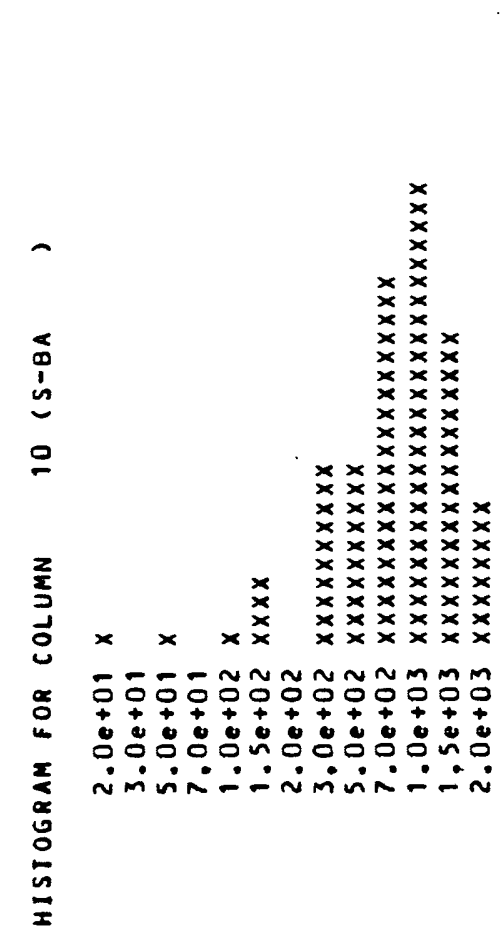

$\infty \sim$

- $\stackrel{0}{0}$

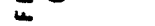
แ $a$

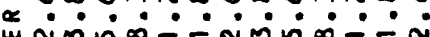

马 w $\sim a \frac{a}{2}$

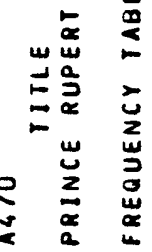

$\bar{\Sigma}, 1,1,1,1,1,1$,

ปะ -

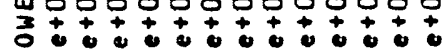

$-\infty 0 \infty 0 m \sim \infty 0 \infty 0 m \sim \infty$

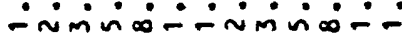

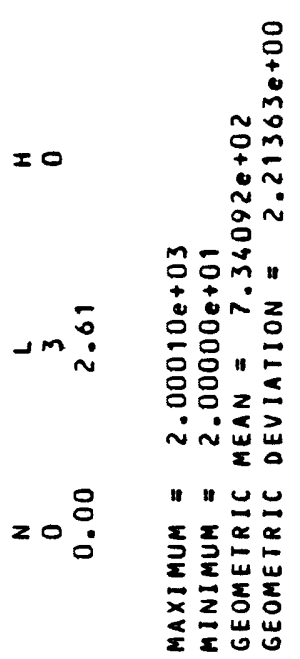




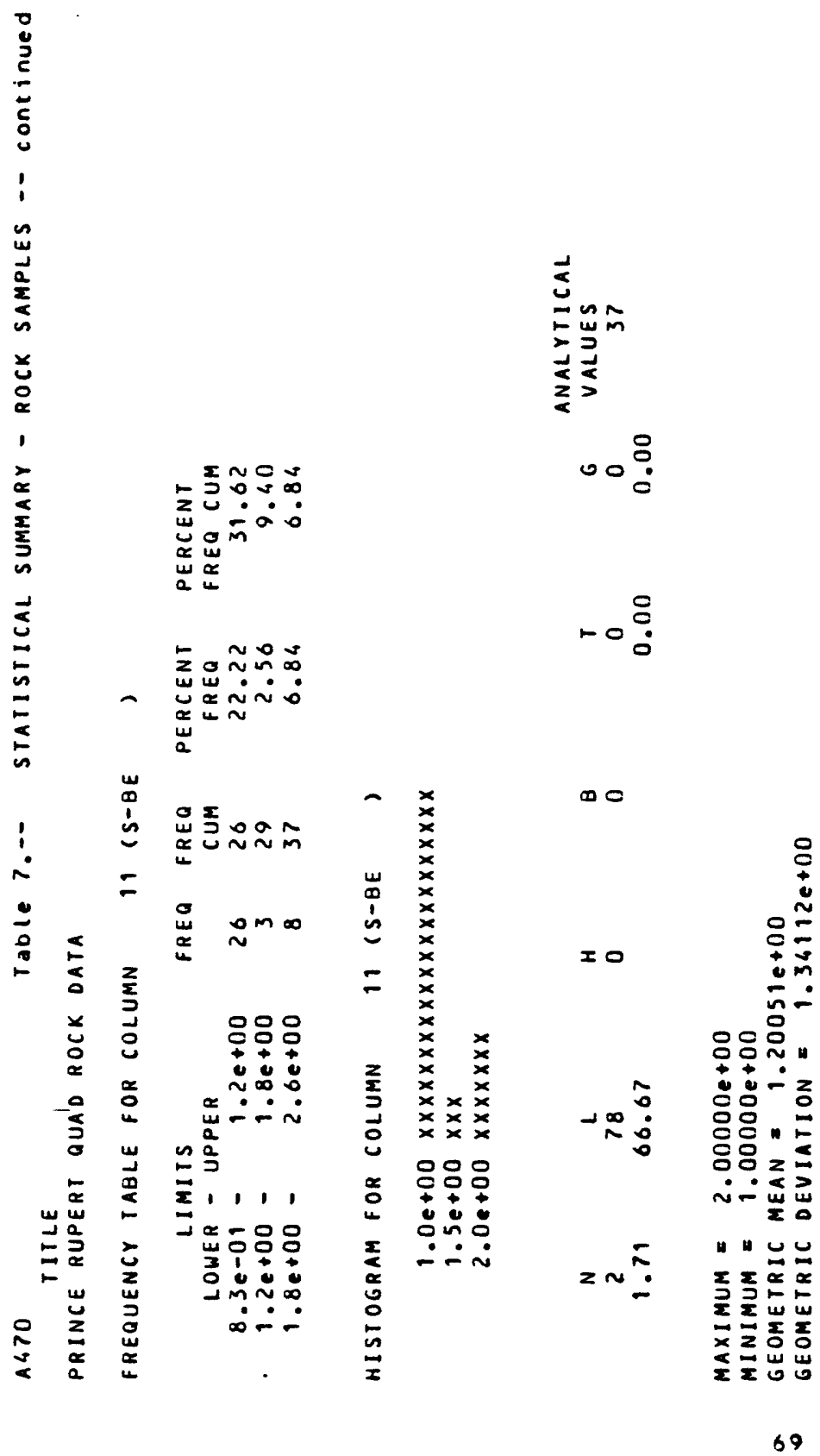


₹oำ

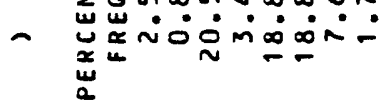

i $\quad a=$

i

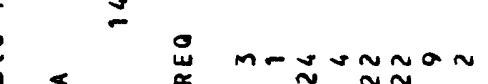

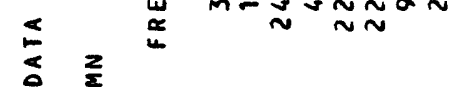

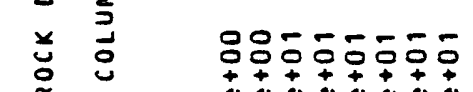

年

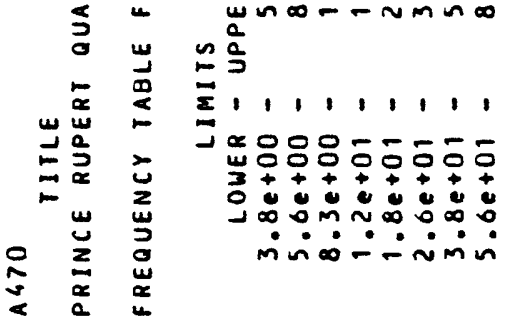

0
$\vdots$
$\vdots$
$=$ $\infty 0$

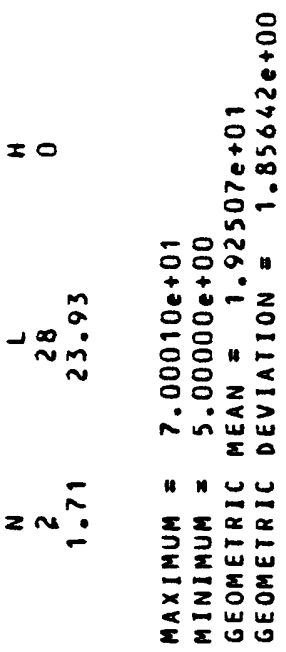




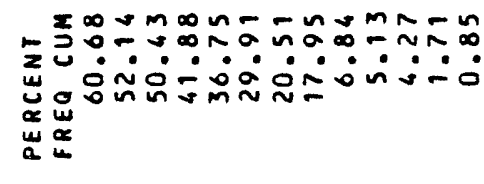

$$
\text { ט } 0 \text { 웅 }
$$

-

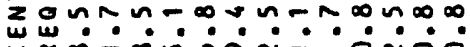

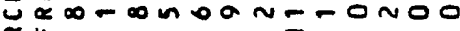
$\underset{a}{\infty}$

in i $0 N 00 \infty-m m N-m,-$ a

$\simeq$

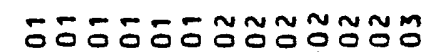

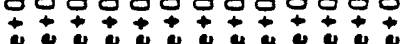
บั:

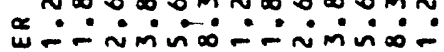
wa $\sim \frac{a}{5}$

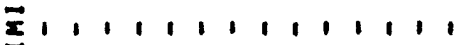

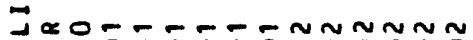
넌우웅ㅇㅇㅇㅇㅇㅇㅇㅇㅇㅇㅇㅇㅇㅇㄴ

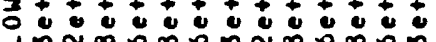

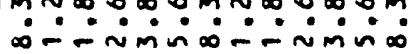

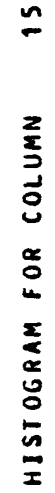

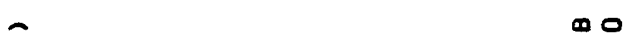

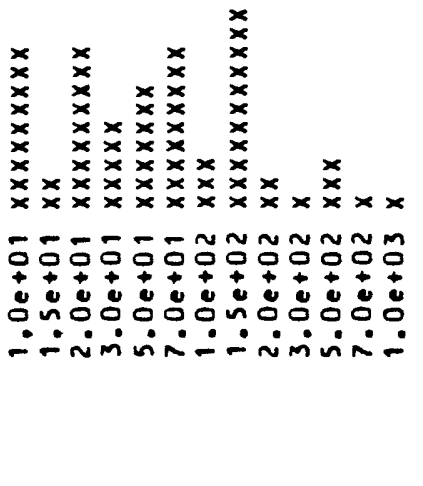

Io

$-0 \stackrel{\circ}{0}$

$\infty 0$

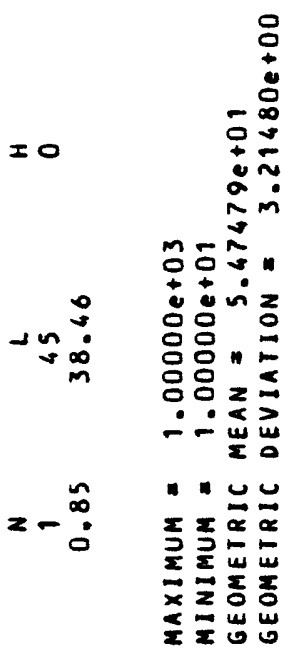




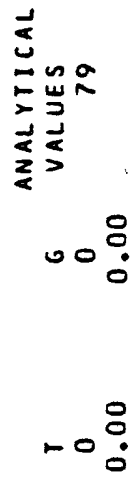

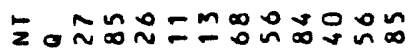

$-\infty \dot{0}$

-

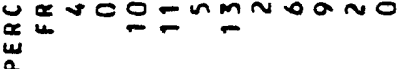

$00 \stackrel{8}{0}$

บ

0

i

แ্ำ

$\stackrel{0}{0}$

$<\underset{\infty}{0} n-\simeq m \infty 0 m \infty=m-$

造

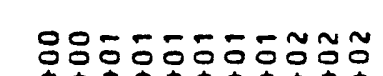

응응ㄷㅇㅇㅎㅁㄷㅇㅇㅠ ڤัต் $\sim \frac{a}{a}$

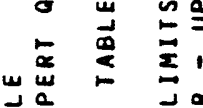

111111111211111

د응ㅇㅇㅇㄷㅇㄷㄷㄷㅇㅜ

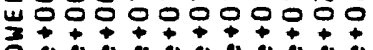
-

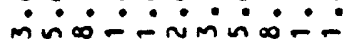

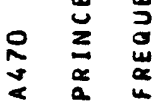

¿

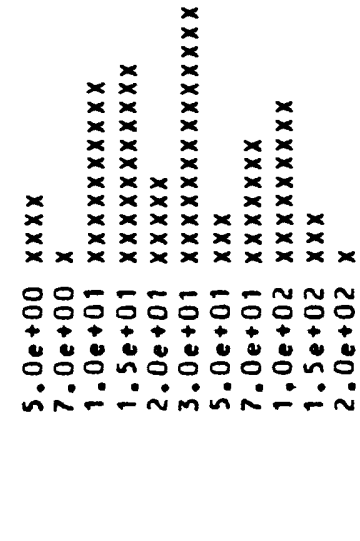

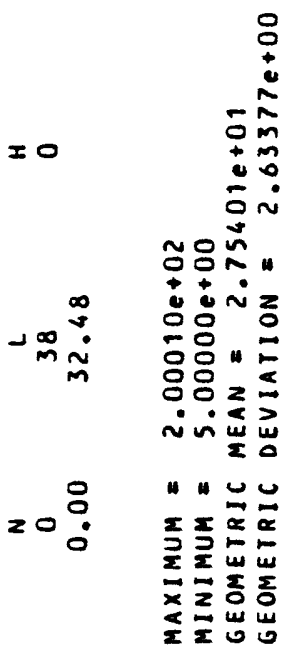



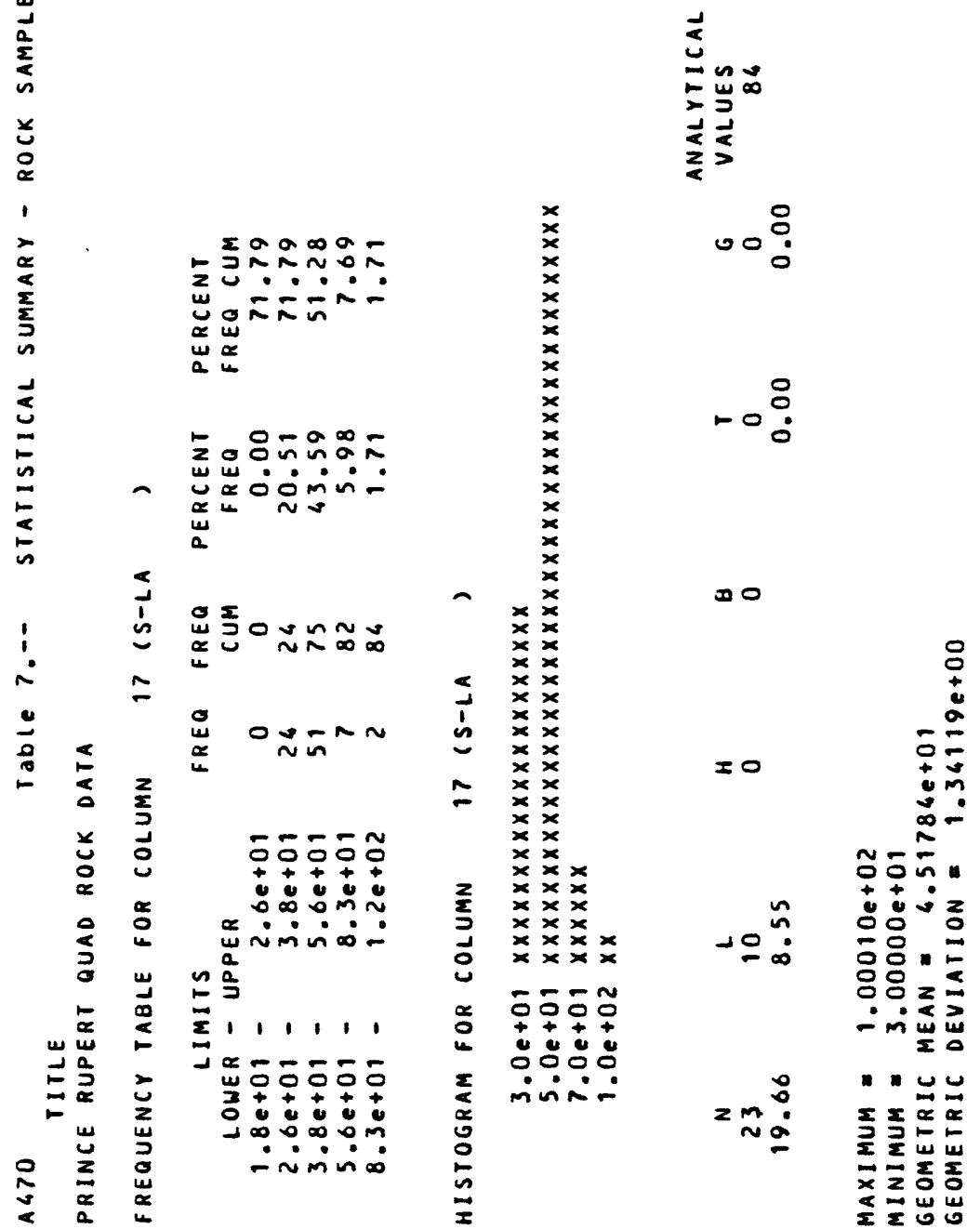


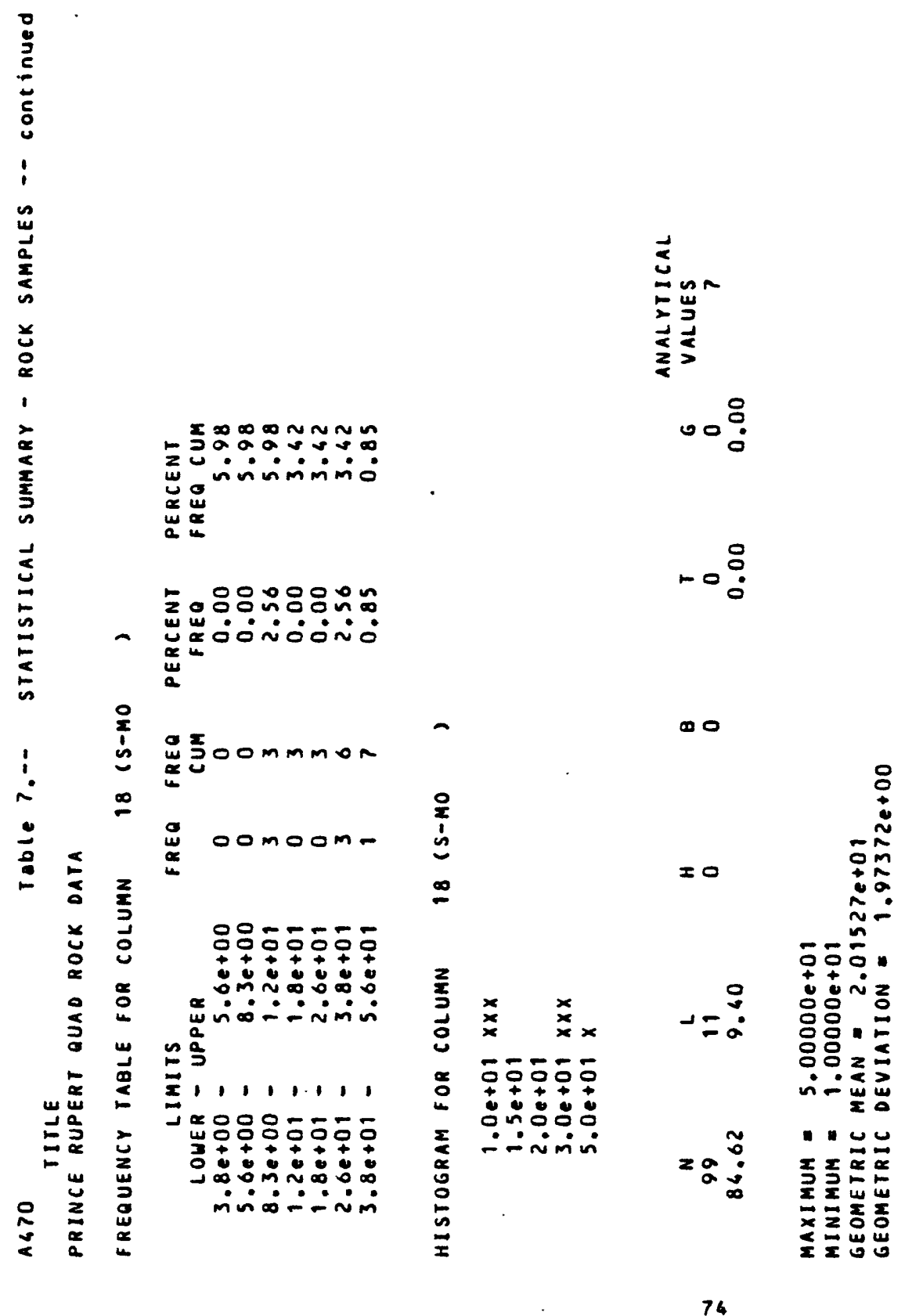




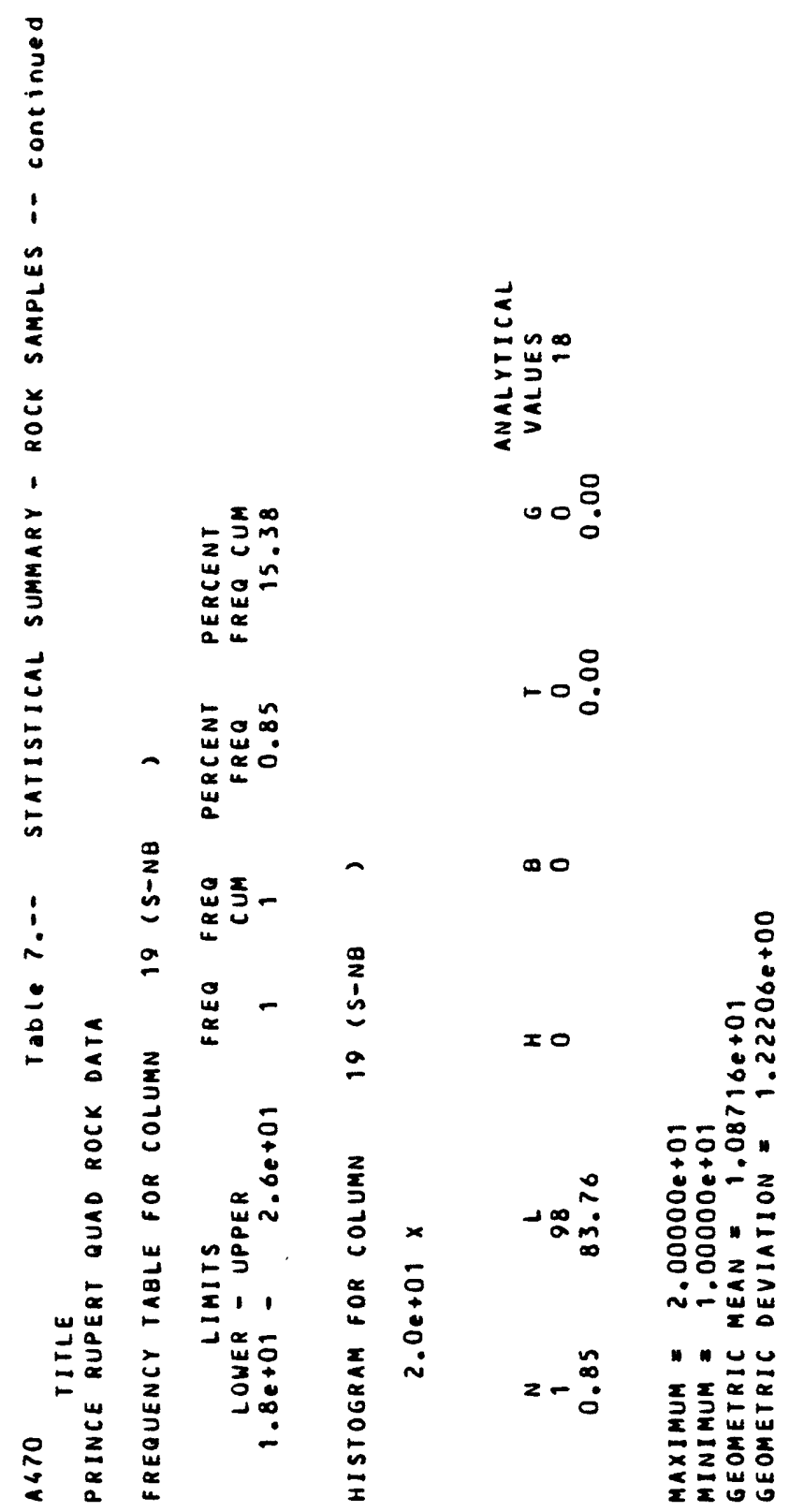




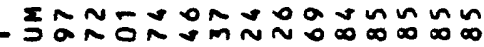
z

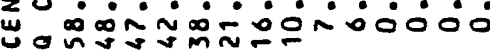

$\underset{w}{w}$

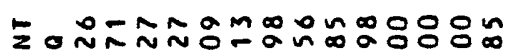

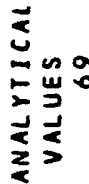

$00 \stackrel{8}{0}$

- 0 :

a
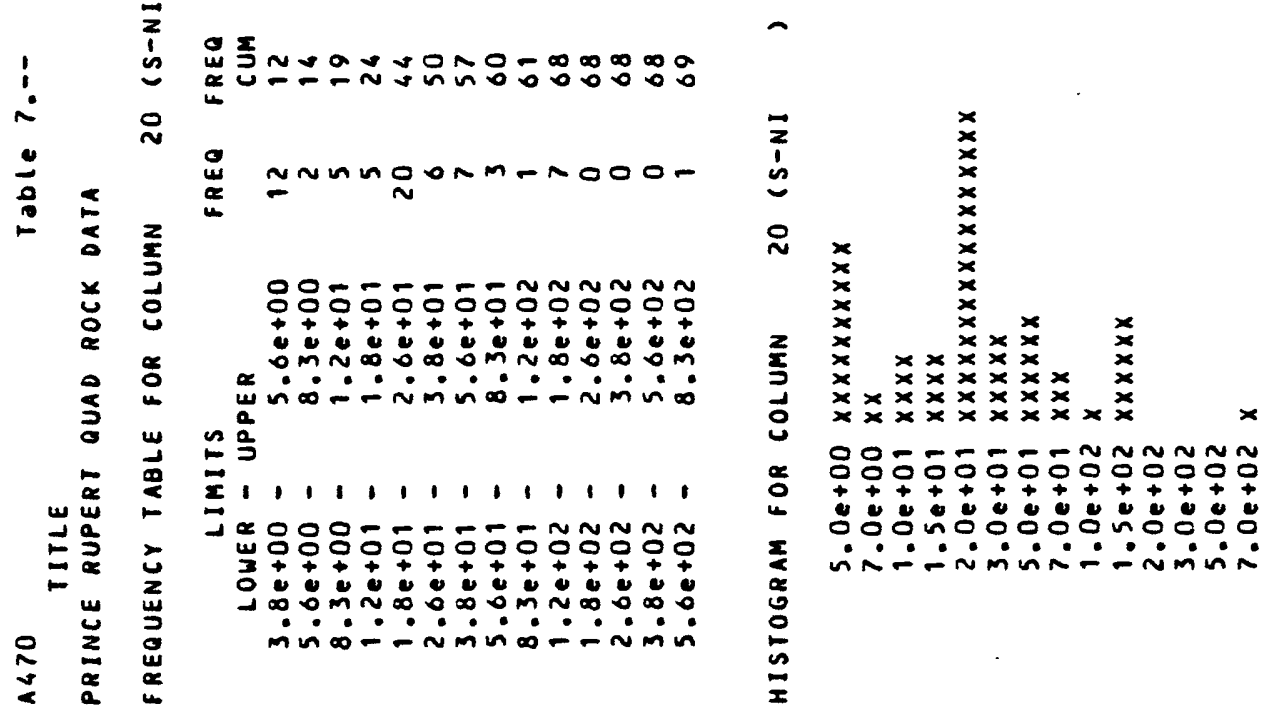

$\infty 0$

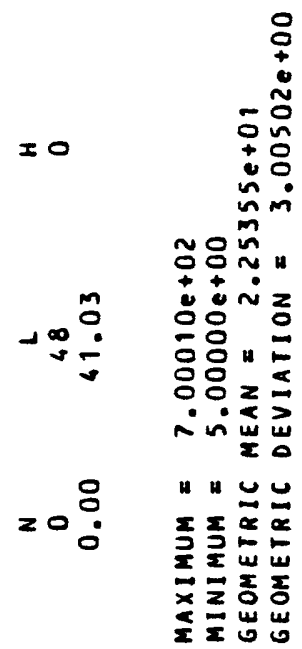



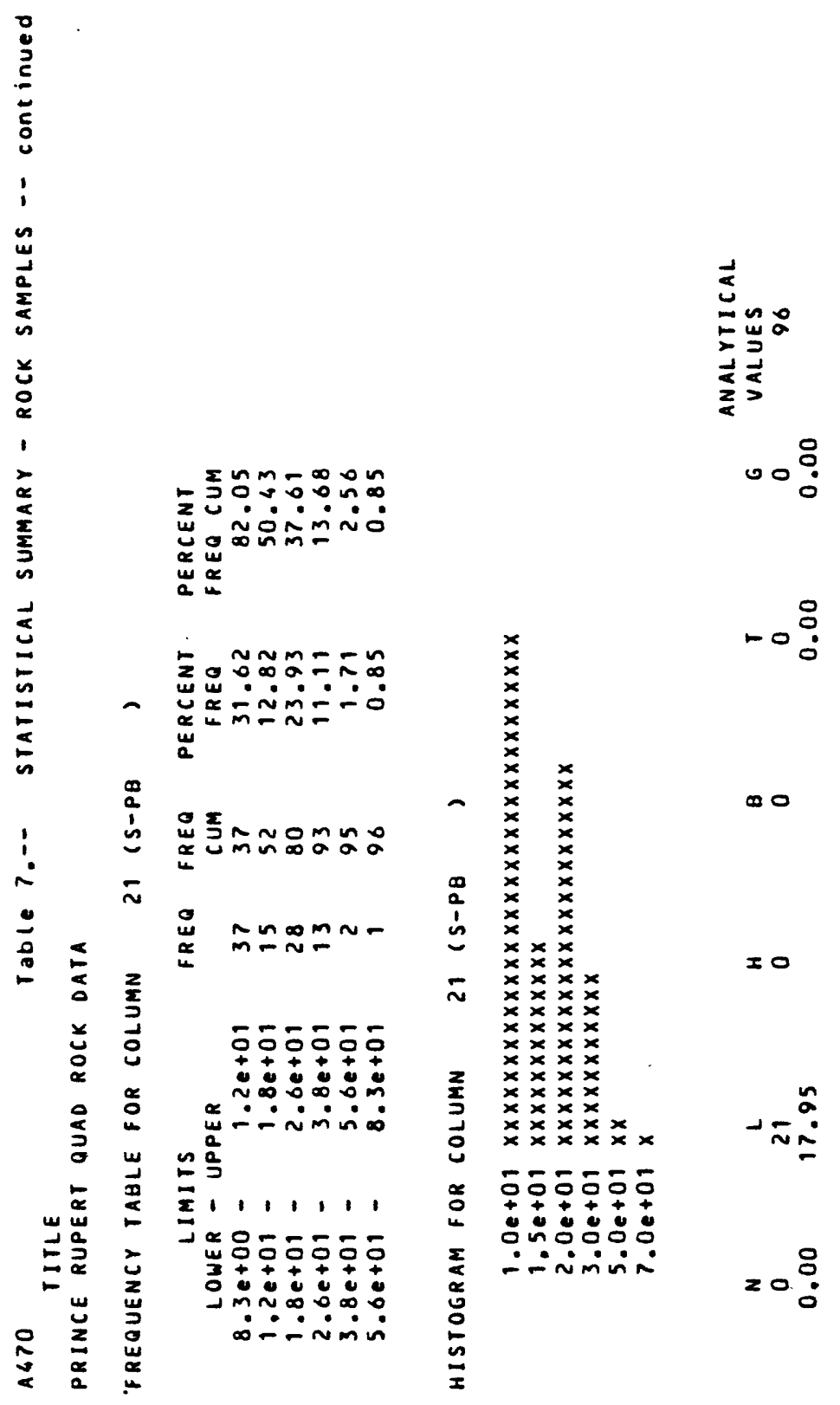

$\infty 0$

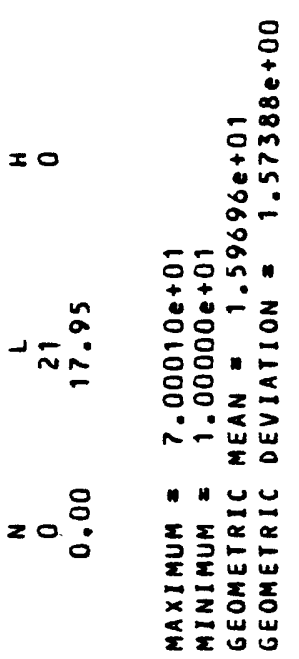




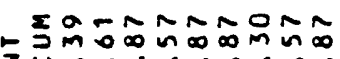

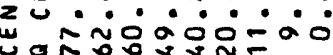
幽

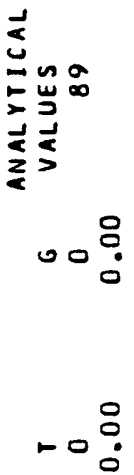

-

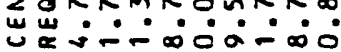
近

a

\section{ํㅗำดก ก

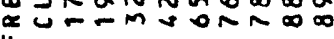

* Nmำ№-

$\stackrel{0}{\circ}$

$$
\text { 妾 }
$$
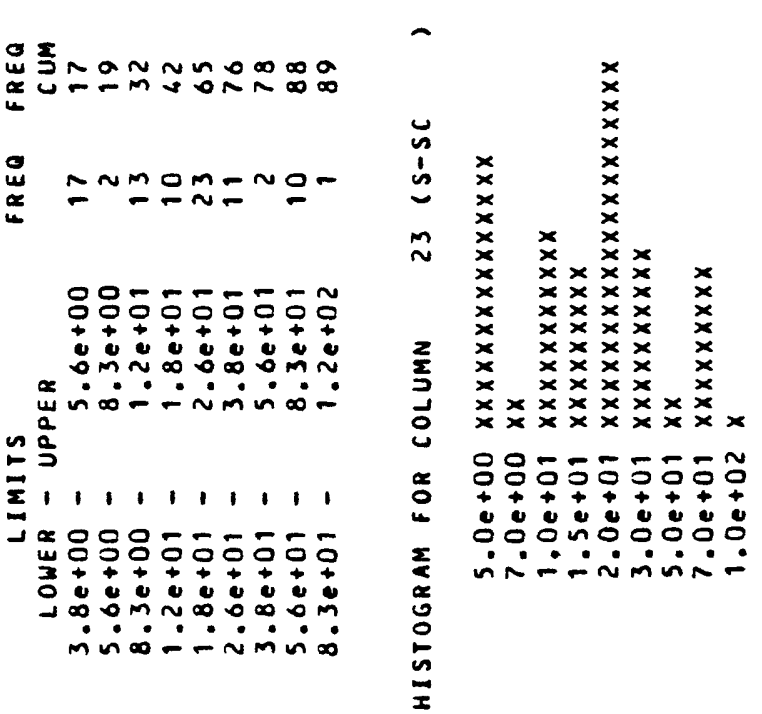

$\infty \sim$

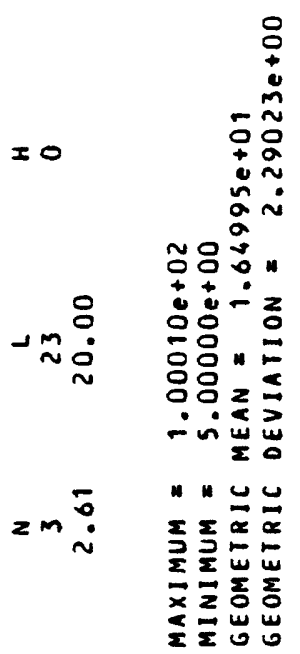



竞

$00:$

-0:

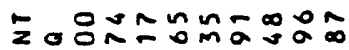

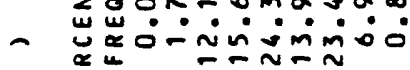
a.
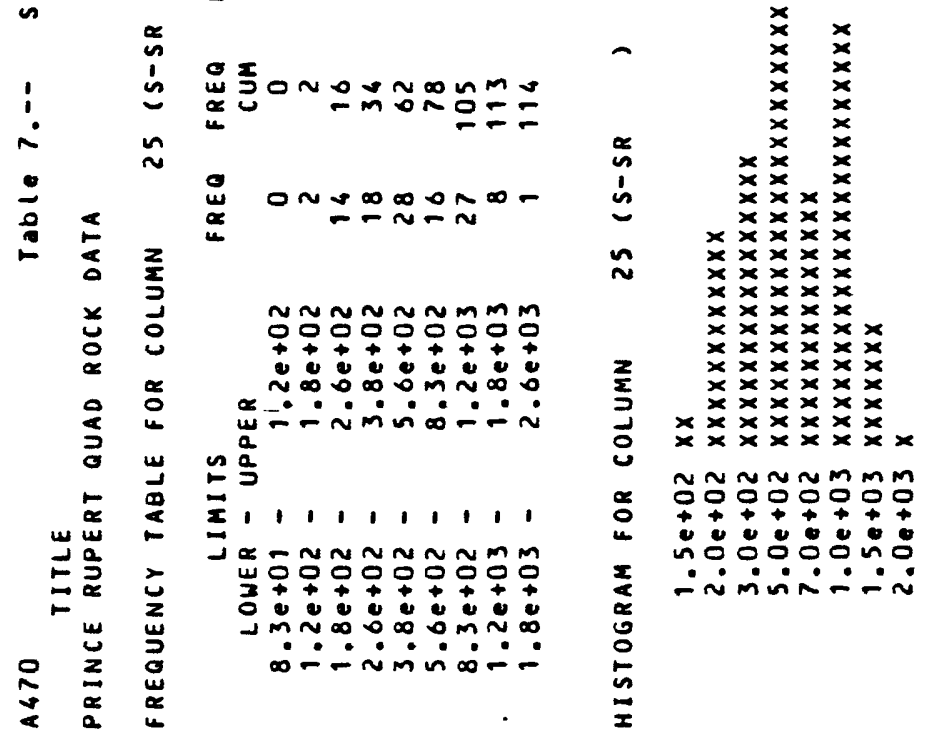

$\infty \sim$

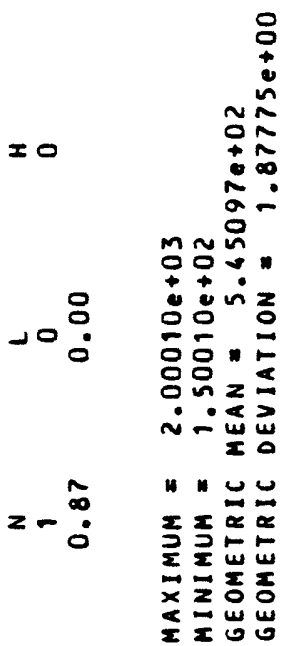



焉

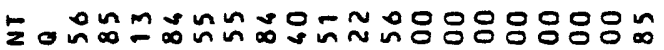

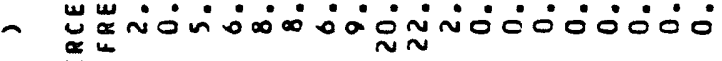
a 晒

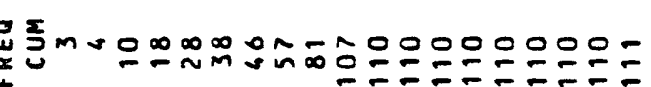

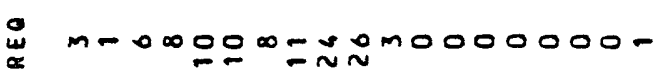

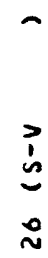

$++1+0.00000000000$ U ヘ⿻ $\frac{m}{a}$ $\stackrel{2}{\frac{a}{2}}$

$\overline{2} 1,1,1,1,1,1,1,1,1,1,1$

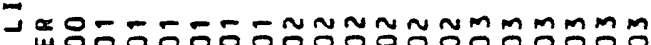

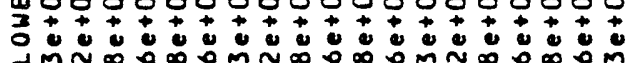

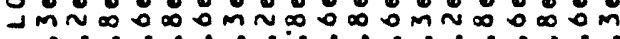

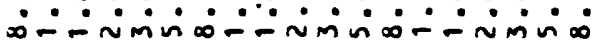

?

$\sum_{0}^{2}$

$\stackrel{2}{0}$ $\sum$

$\because \underset{\Xi}{m}=$

$\sum$

ט $0:$

$-0 \stackrel{\circ}{\circ}$

$\infty 0$

Io

5
0
0
0
0
$\vdots$
$\frac{1}{3}$

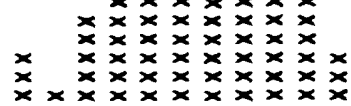

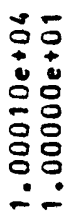

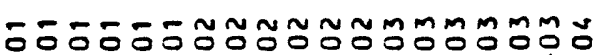

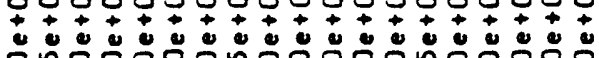

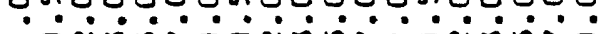



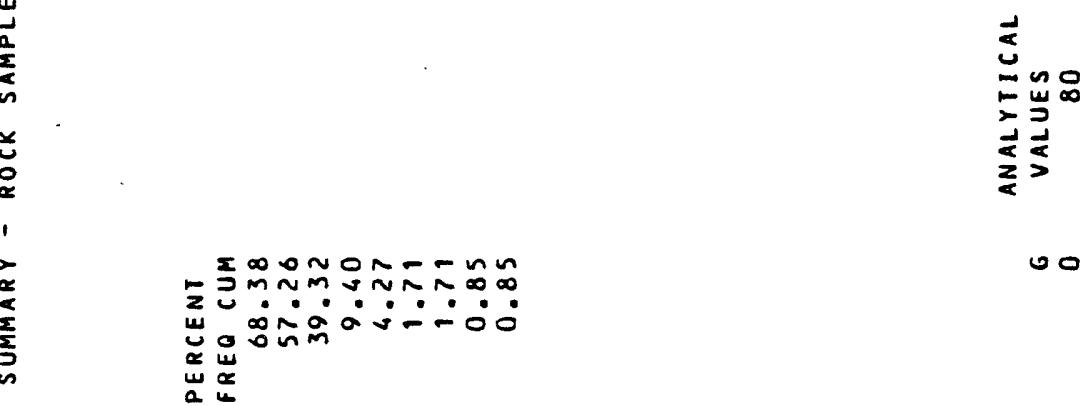

$00 \stackrel{\circ}{\circ}$

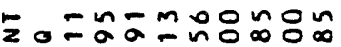
ü

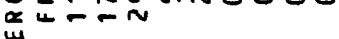

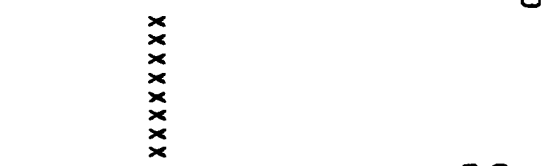

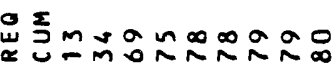
$\stackrel{\infty}{\sim}$

㟧 m̃ñmoror

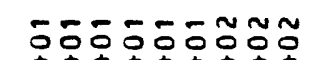
t.t! t+t! $\sim \cdots \infty \infty \cdots \div \infty !$ 药

-

$\infty 0$

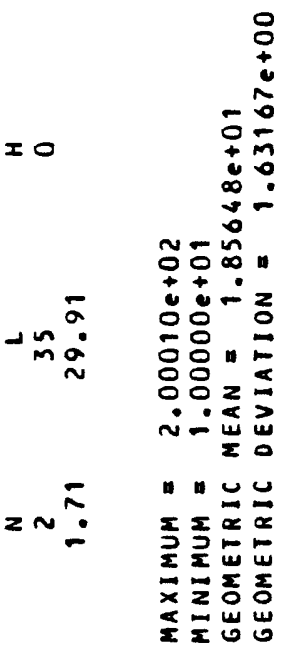



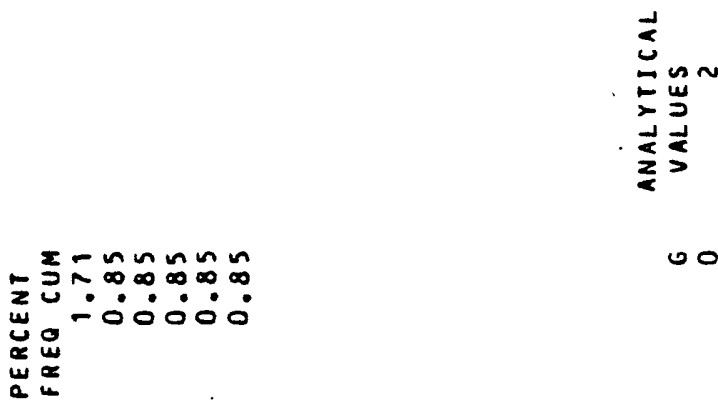

5

₹ด

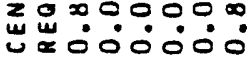

赵

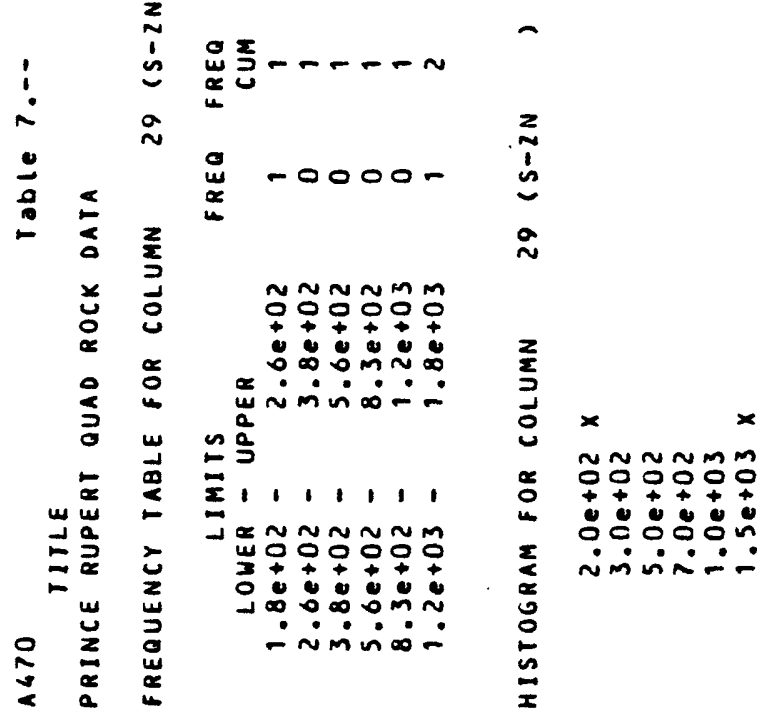

$\infty$

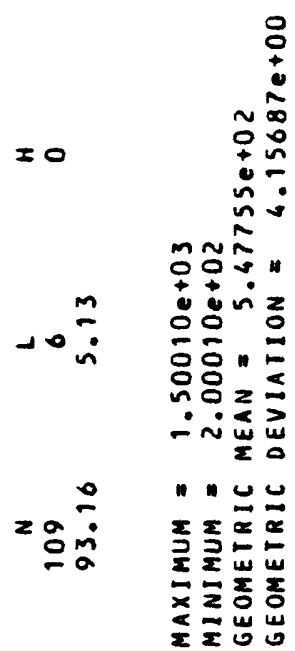




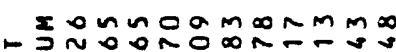

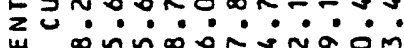

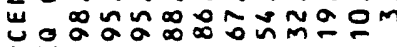
$\stackrel{\sim}{\sim} \underset{\sim}{\alpha}$

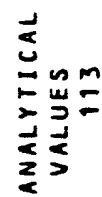

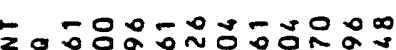
ราำㅇำ

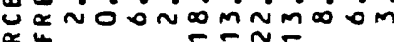
学

i

$$
\text { 원근 }
$$
울

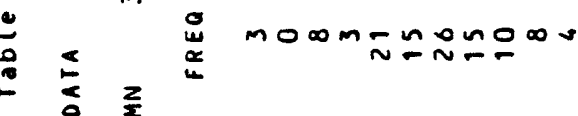

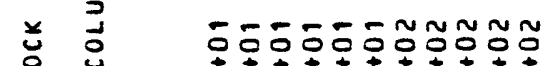

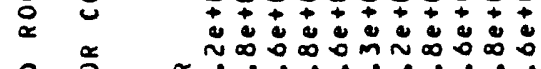

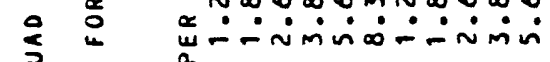

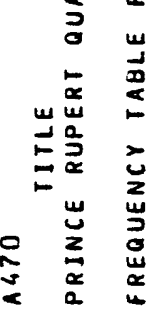
$\approx \frac{a}{2}$

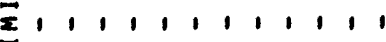

ม으으드ำกับ

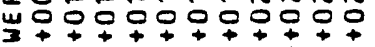
ป $\infty \div \sim$ imin $\dot{0}-\dot{m}$

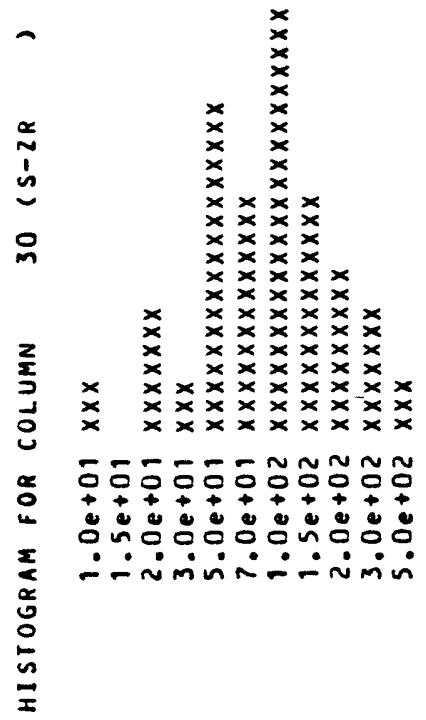

$\infty \sim$

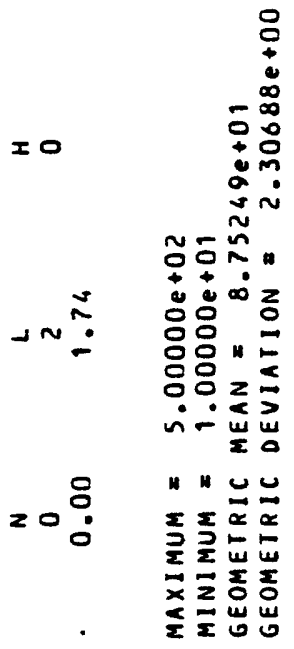



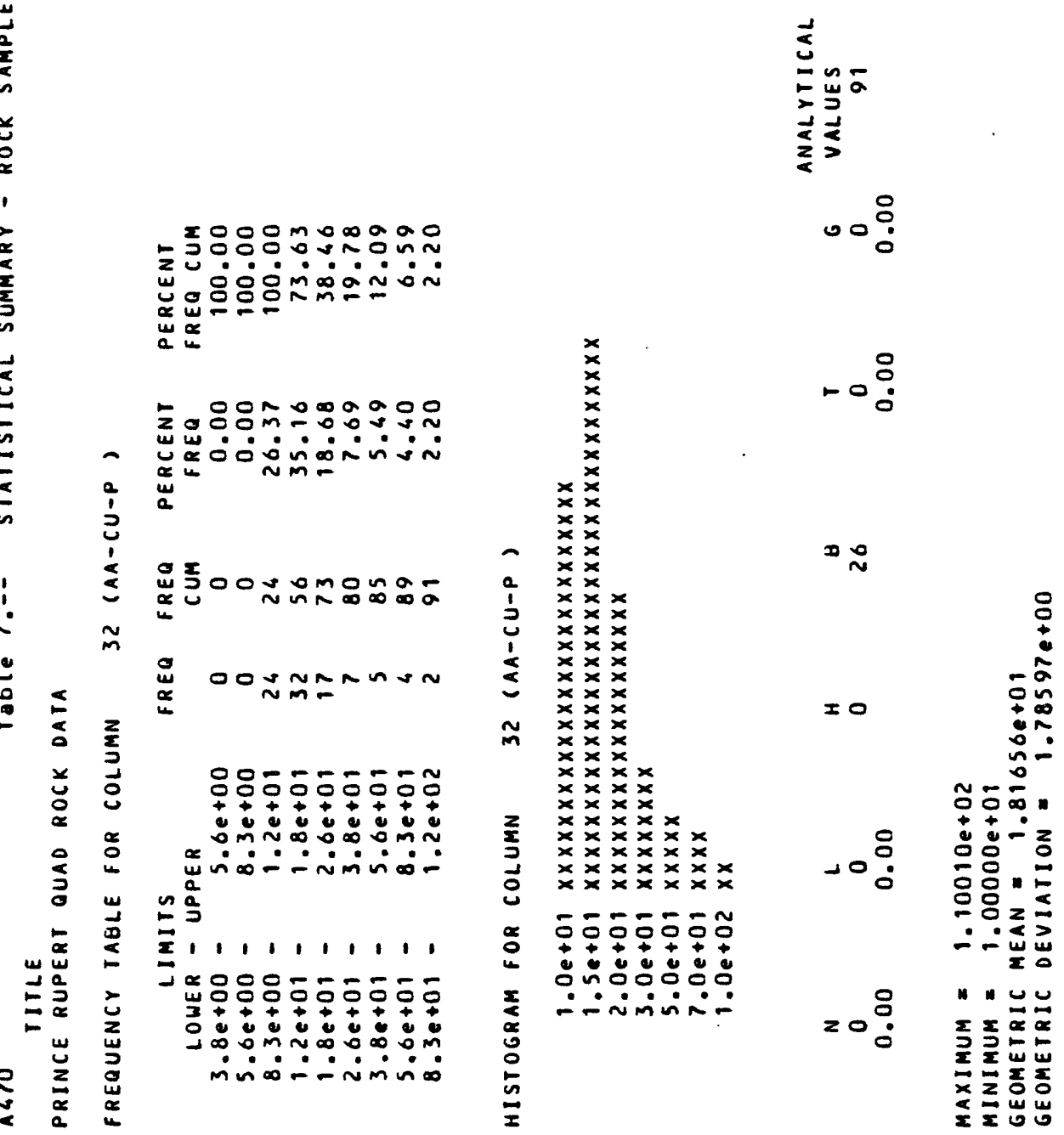


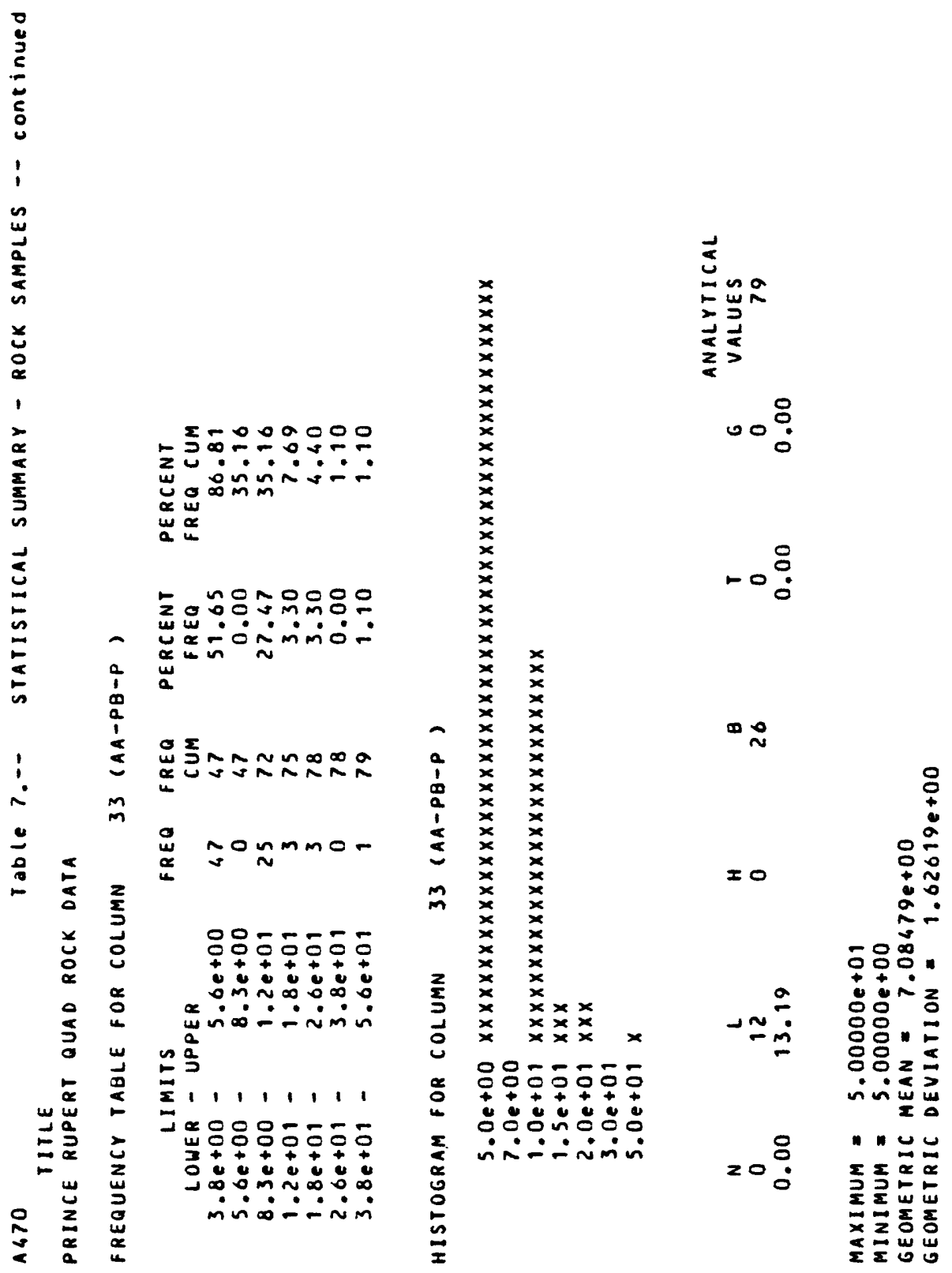




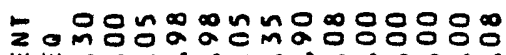
-0:

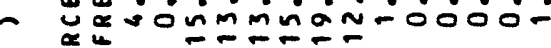
$\div \quad w$

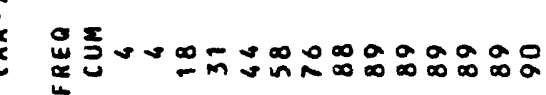
in

i

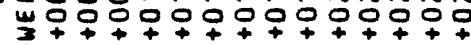
ơ:

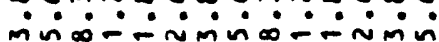

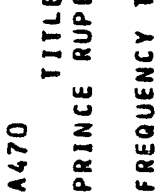

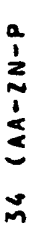

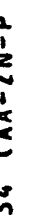

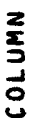

\section{$x$}

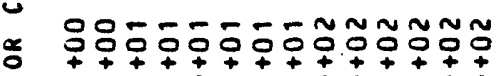

운

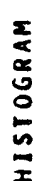

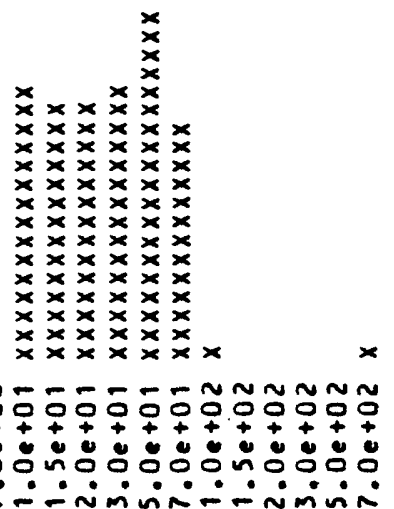

no

$\infty$

$=0 \quad$

웅ํ.

$-\stackrel{\infty}{-}$

워응

항을

응ํㅊ

柁出岕

$2 \sim \stackrel{n}{n}$

" 


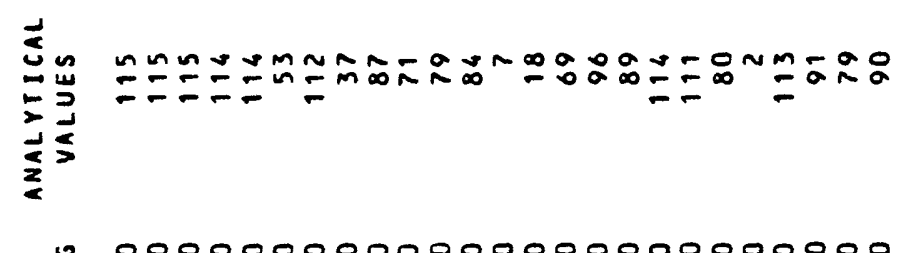

- 0000000000000000000000000

- 0000000000000000000000000

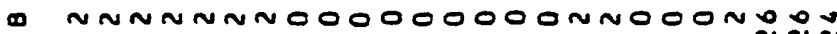
N N

I 0000000000000000000000000

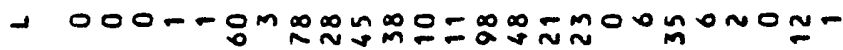

$=00000 N 0 N n-0 m a-00 m-0 N g 000 n$

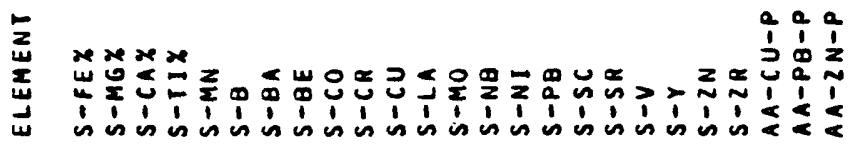




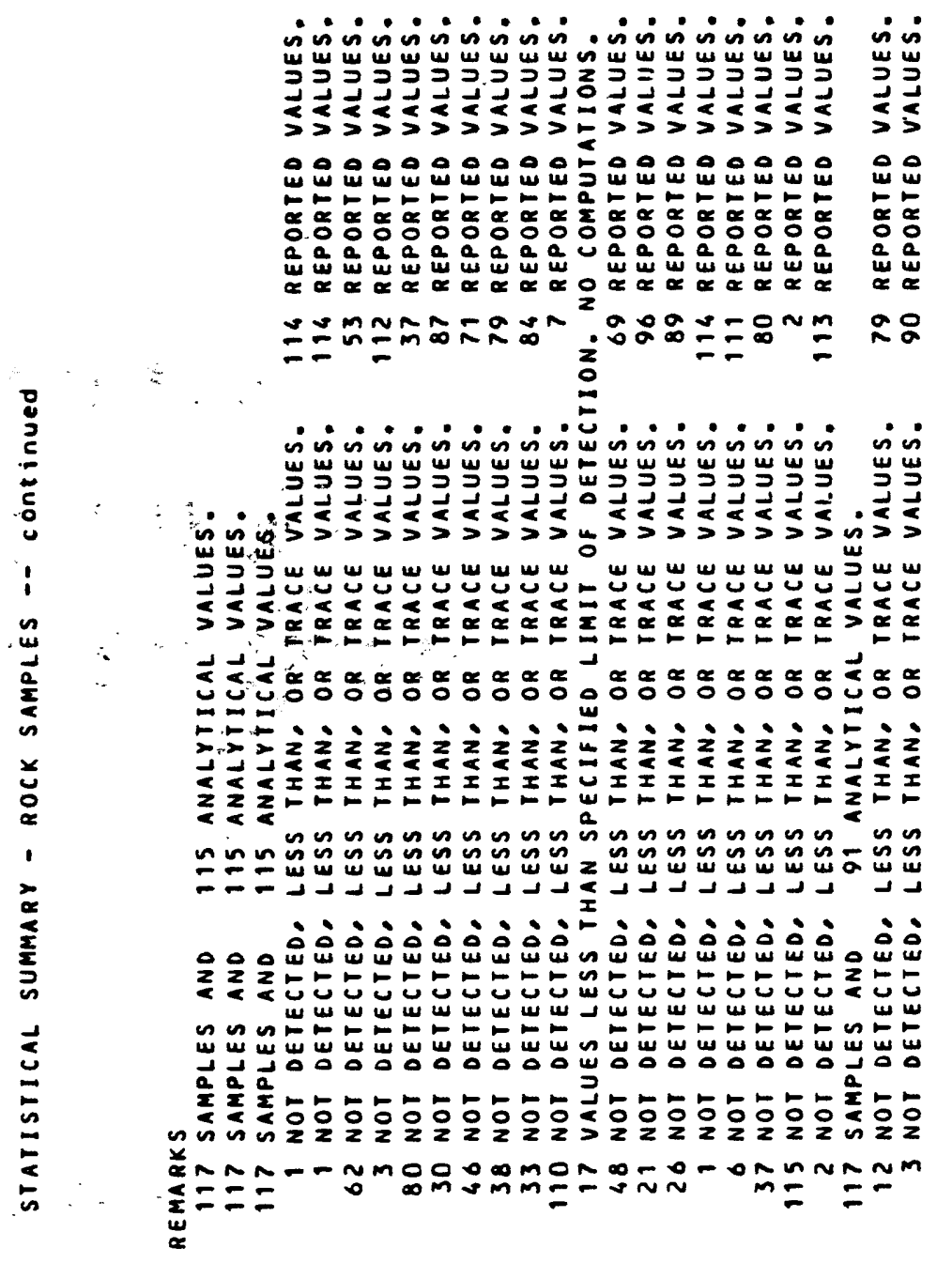

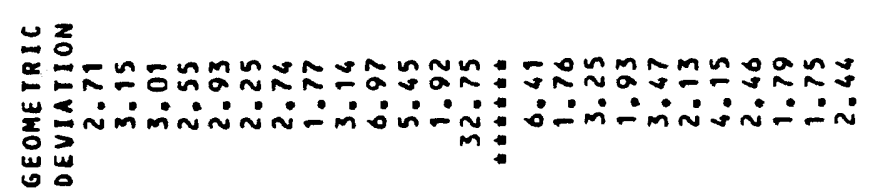

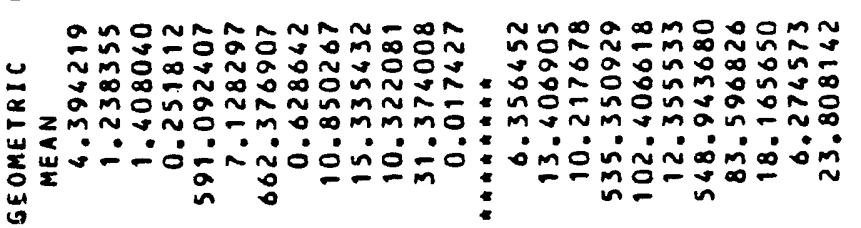

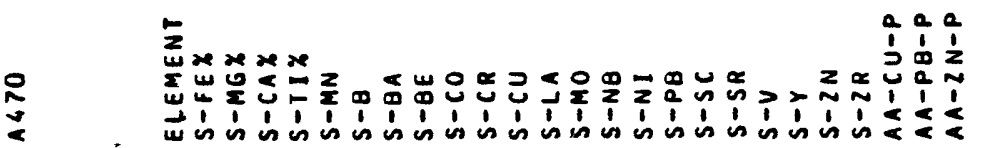




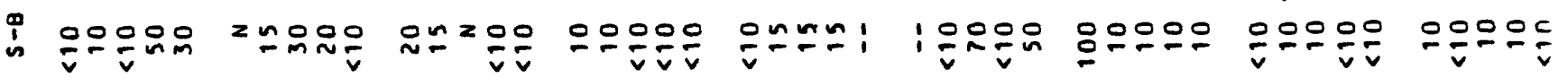
变 n

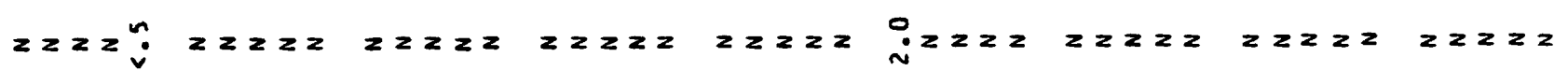

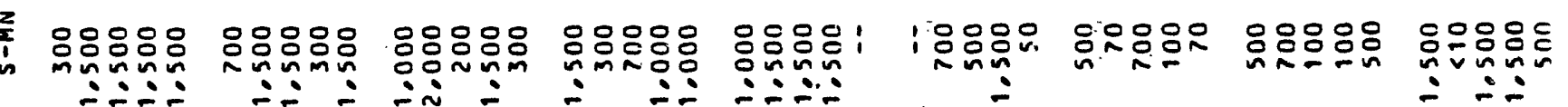

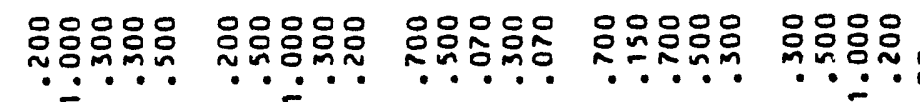

응ㅇㅇㅇㅛ $\therefore$ जin்:

응ㅇㅇㅇ요웅 $\because \therefore \dot{~}$

옹응응응 nin ni-

\section{0}

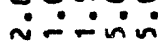

응용용 ninim

\section{용ㅇㅇㅇ \\ inis.}

응용융ㅇㅁ miñ்

영웅요 ini

옹옹용요 m-ini

응옹응요 的丘 운.

00000 mon்문

90000 nioninis

00ํำ 0요.

00000 onió

00001 inivi inini 웅으옴옴.

읐웅음옹옹 ํํํํํำ

응옹응요옹 응유믄

\section{9.}

응융웅 $\because \therefore \dot{m}$

운유운운 กำ

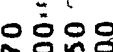
군? (1)

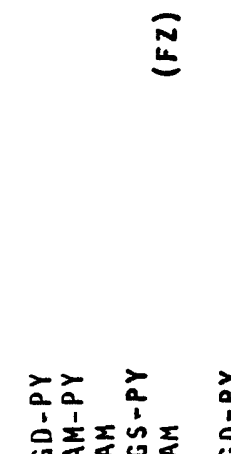

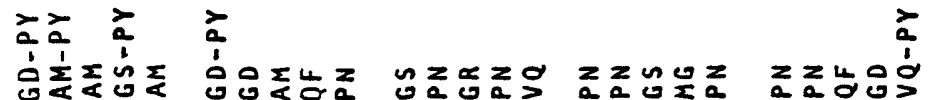

ป

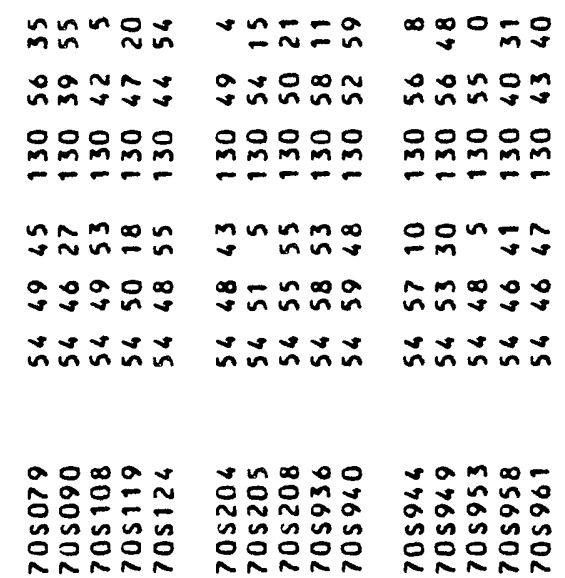

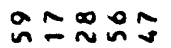

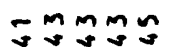

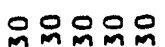

끄므므

กะกละก

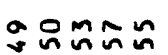

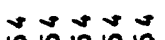

ำะก

ำงำ

웄윰임요 ํㅗㅁำ

mons n

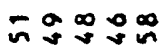

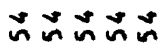

$\infty ー \geq \infty \bar{n}$

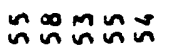

ogooo

mmmpm

जึะmo

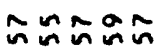

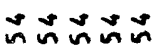

ํㅜํํำำ

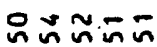

mmmm

nMg:-

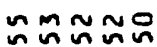

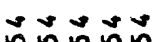

웅ㅇㅇㅇ opeo

กำ

00000

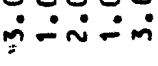

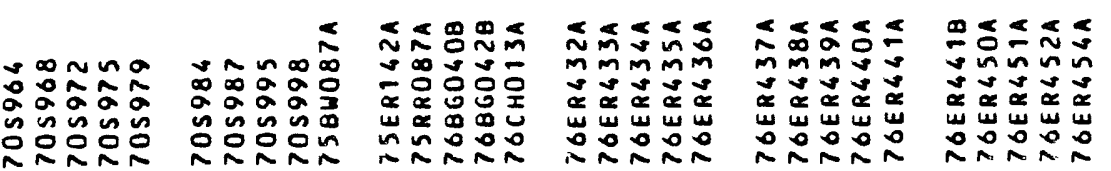

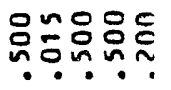
$\dot{\sim} \dot{\sim} \dot{-}$

동응응 $\dot{m} \sim \dot{\sim}$

$0 \sim 000$ $\because \dot{0} \dot{\sim}$

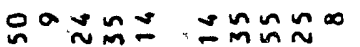

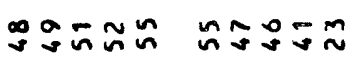

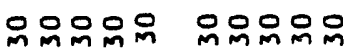




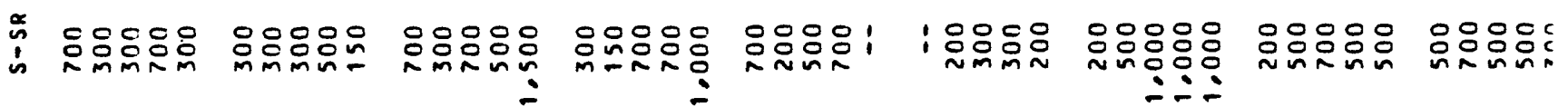

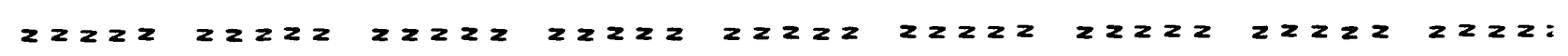

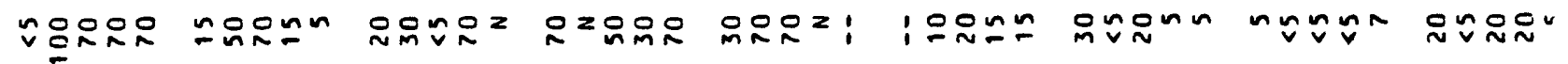

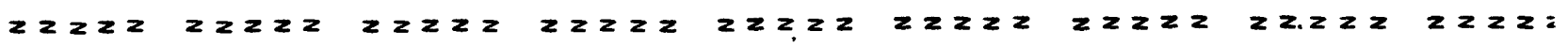

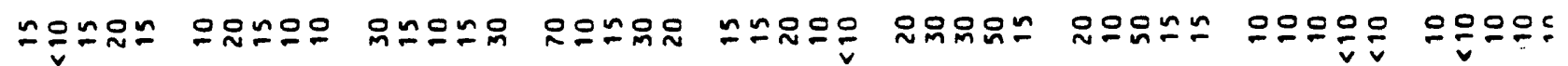

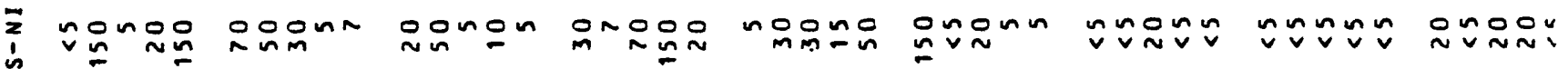

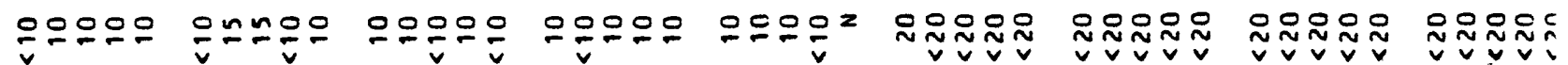

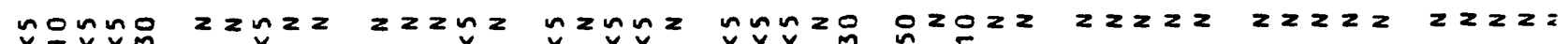

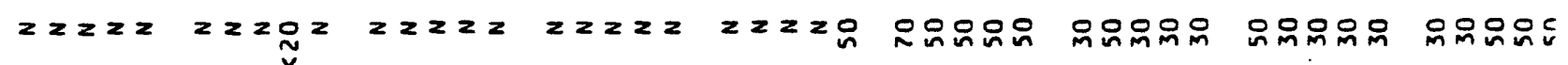

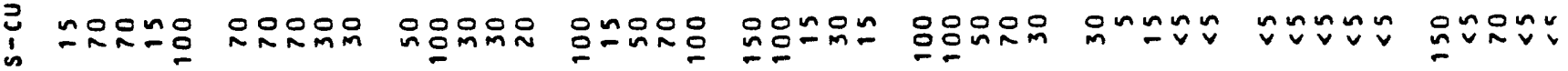

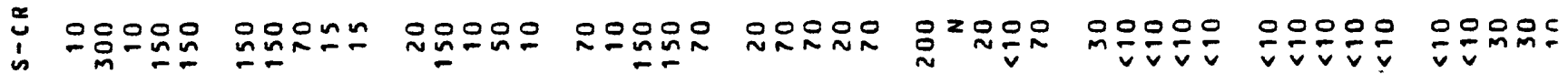

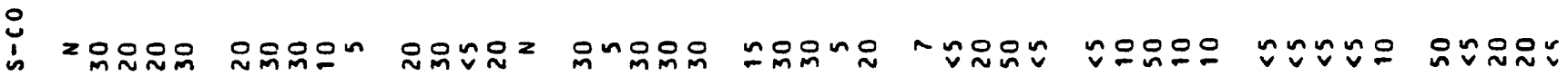
í

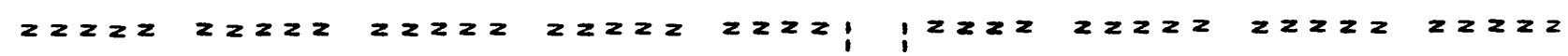

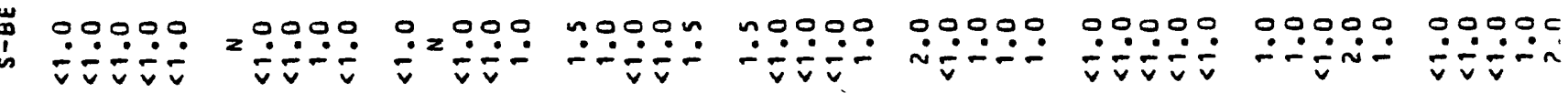

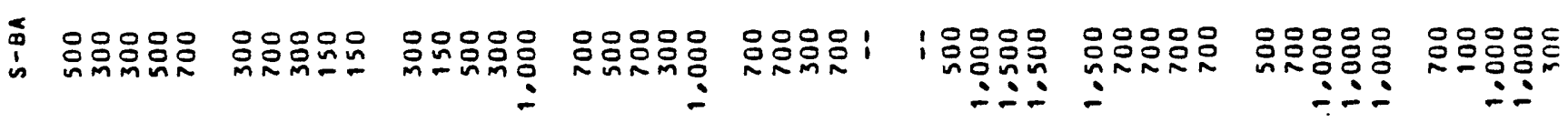

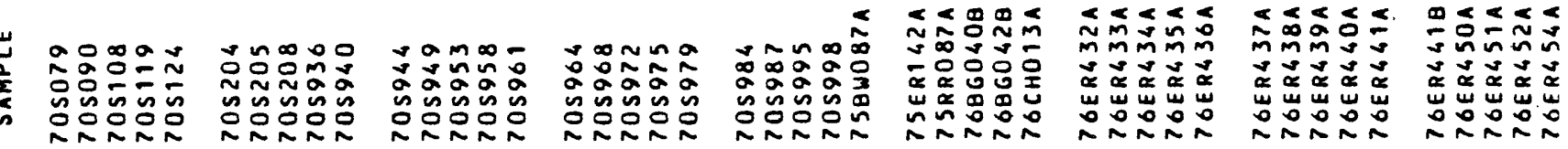




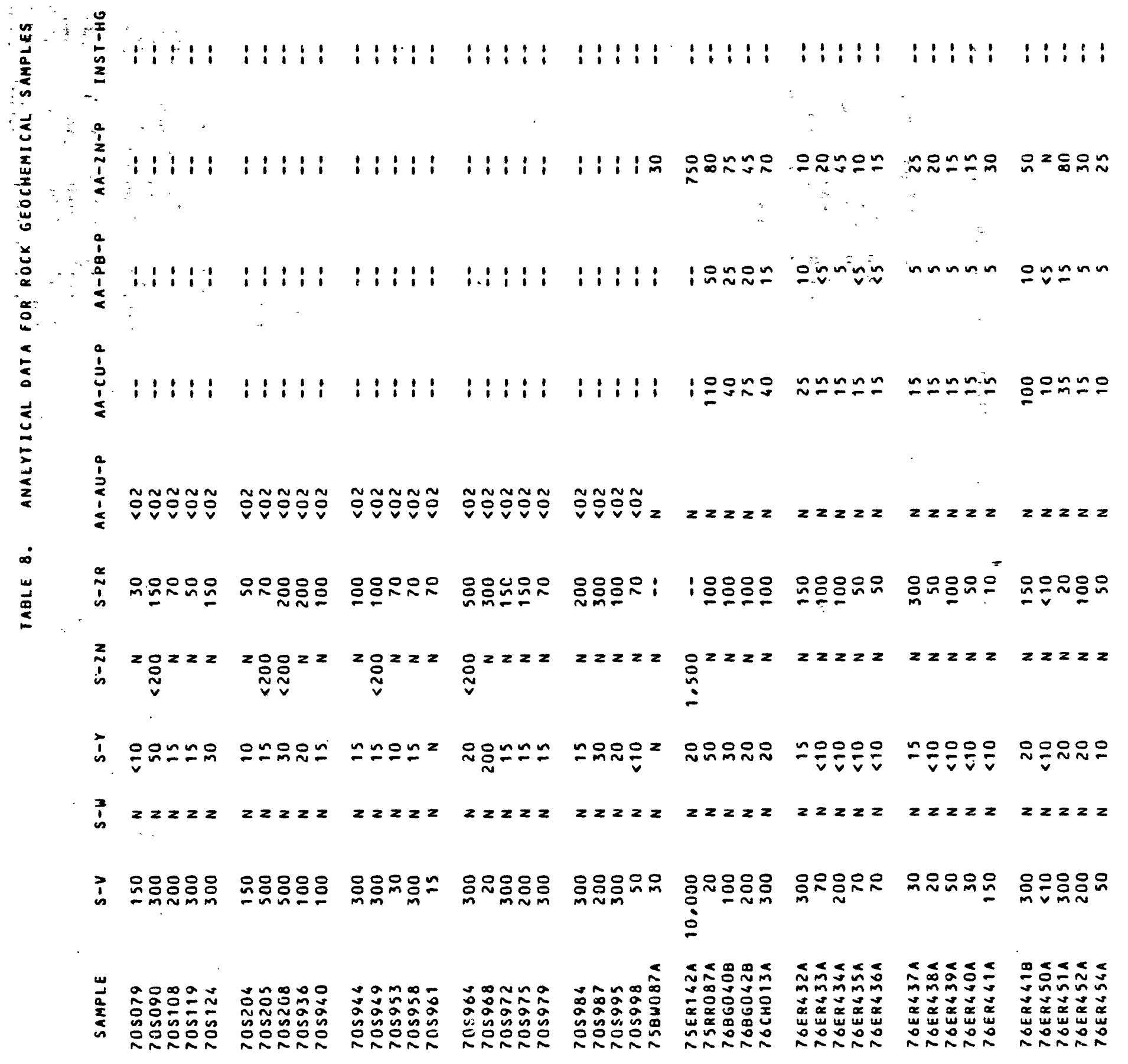




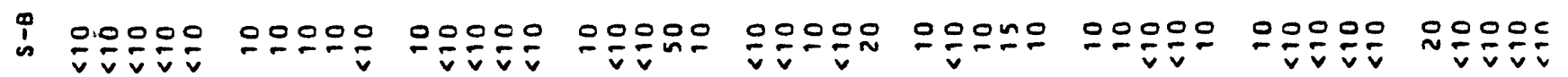

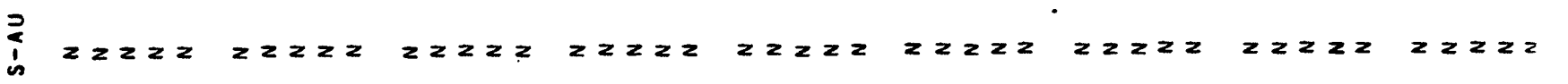

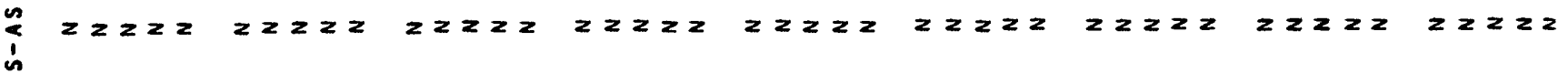

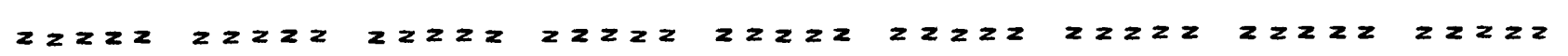

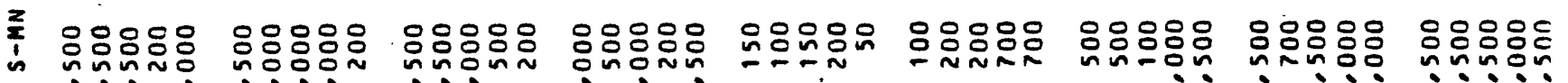

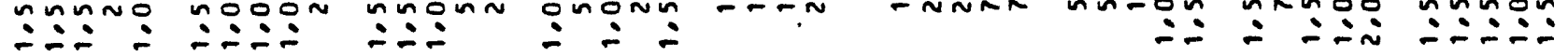

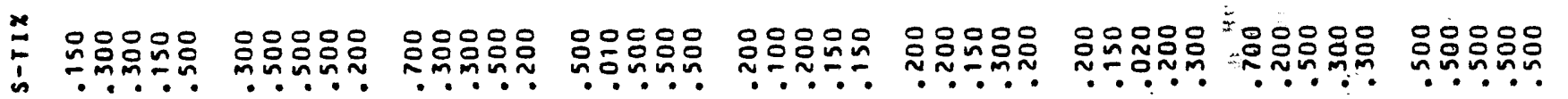

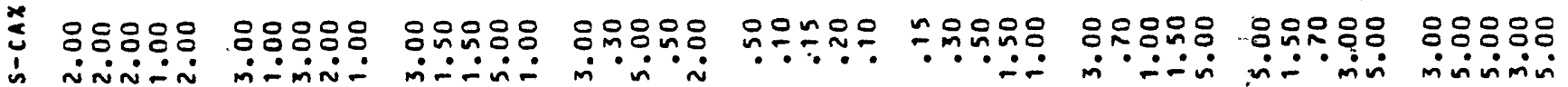

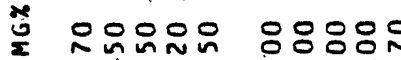

$\because \div$ - minin.

응응응용

웅옹응응응

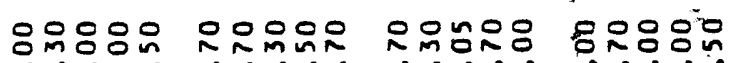

응웅웅웅 $\dot{n} \dot{\sim} \dot{-1}$ inim-i $\dot{-1}$

in in

inimin

00000

00000 ヘ

வீำ

00000

ónimi

00000

oogun unnoo

onnoo

00000

00000 ¿miñ

$\ddot{\sim} \dot{m} \div \dot{-} \therefore-\dot{0} \dot{0}$

í: min

inं

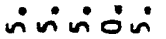

$\infty$

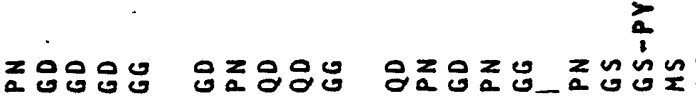

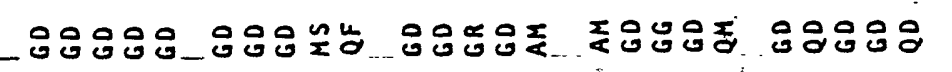

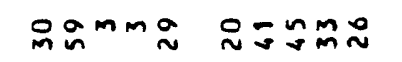

กñ

ñon

nninnin

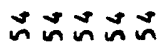

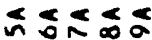

nnnin

まする゙す

热

0000

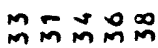

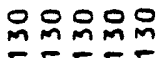

nnOñ

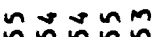

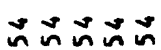

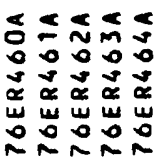

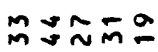

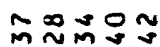

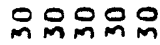
-....

กำํำ

品的守舟品

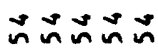

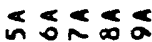

ก. 000

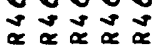

س NNㅇ

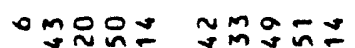
动的的 00000

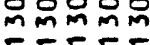

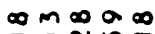
ñnn

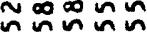

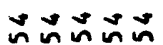

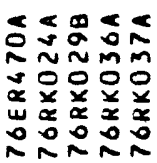

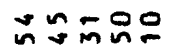
goning

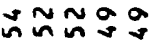

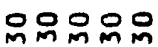
ㄱ-

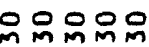
mำ는

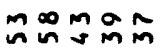
的角的品守 $\infty m-n \tilde{n}$ ? $\infty$ min

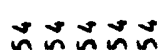

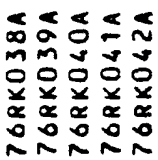

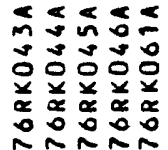

눙유 asonn 00000

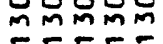
$=0+\infty \infty$

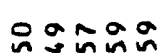

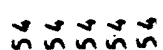

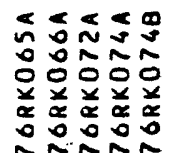

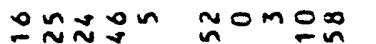

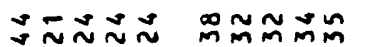
00000 MmMmm $\because n \infty \sim 5$ 串昂的路

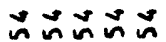

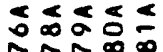

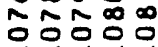

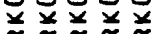

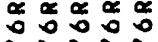

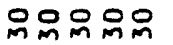

$0 \sim 0 m 0$ บกำ

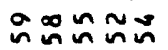

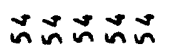

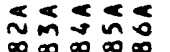
$\infty \infty \infty \infty$ 우웅요요 政 

용용요

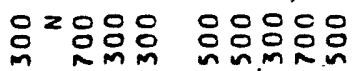

응응음음

n!

음윰유

웅용유

요요

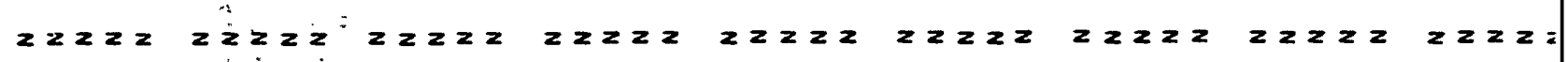

ooond oo 오웅요 ๓ะฐュท nะทำ

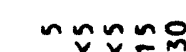
드눙응 옷웅의 Q

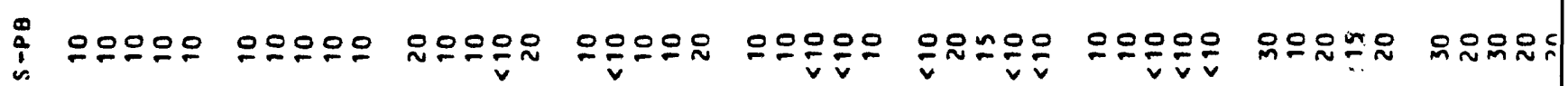

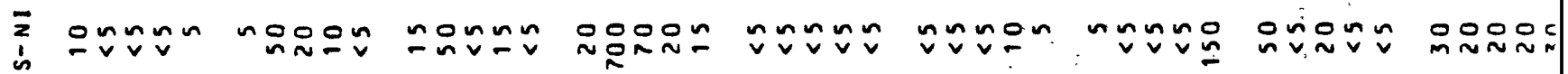

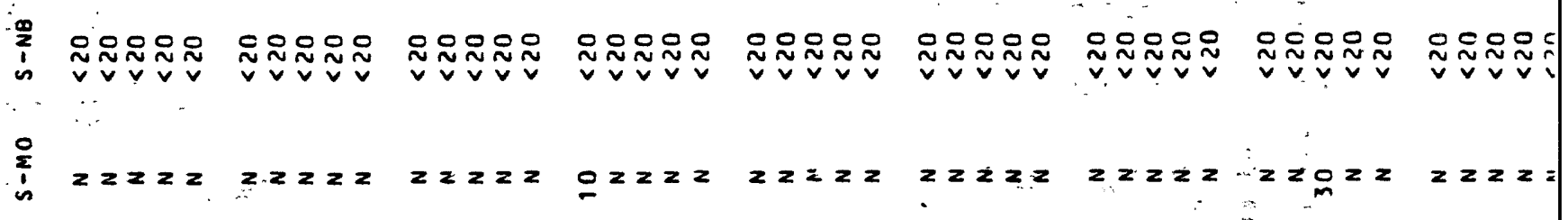

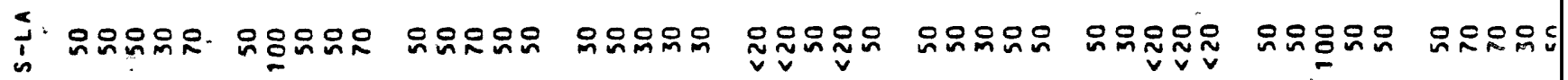

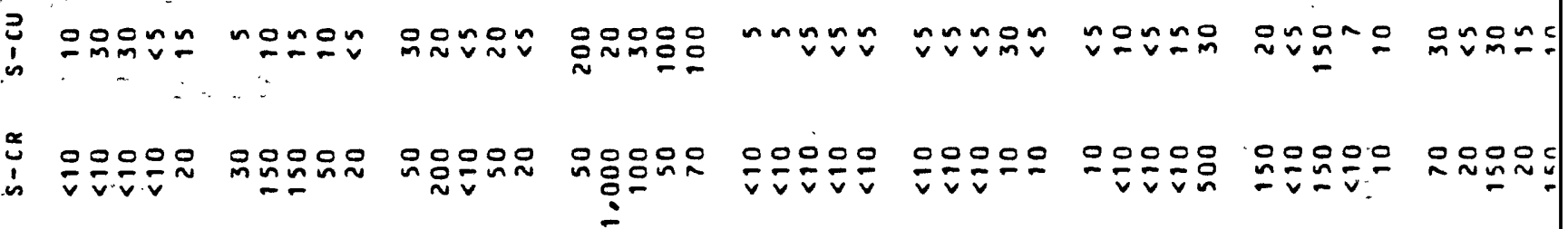

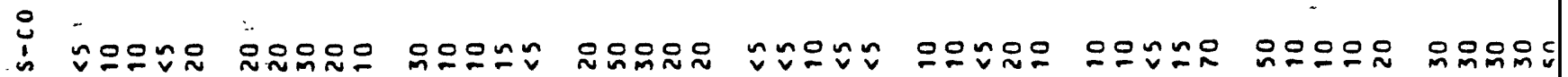
$\infty$

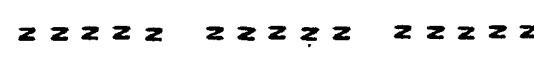
22222 $2 z 2 \geq 2$ $z \geq z 2 z$ $z \geq z \geq 2$ $z z z 2 z \quad 2 z 2 z$

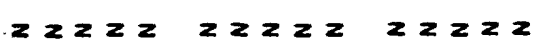
$2222 \cdot 2$ 2222222222 $2 z 222$ $2 z 2 z 2$

$\$ 0000000000$ $\dot{v}-\dot{\sim}$ $\div \div \bar{v}-\dot{v}$

00000

00000

00000

00000

00000

00000

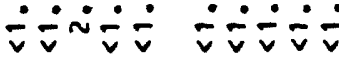

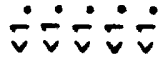

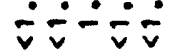
$\div \dot{v} \div \div$

¿ 옹옹ㅇㅇㅇㅇㅇㅇ 응웅응응옹 ㄴํㄴ? 읏응음욤은 옹응ㅇㅁㅇㅇㅇ 은응은

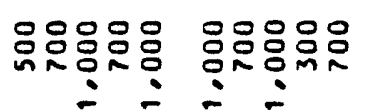
은응응은은 는

옹옹윰윰드 $-\infty-$

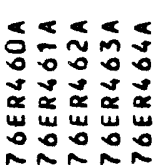

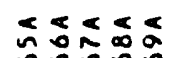

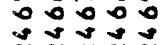
$\alpha \propto \alpha \approx \alpha$

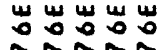
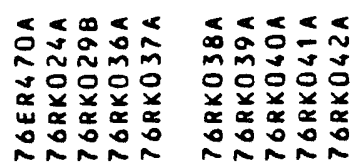

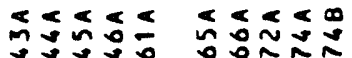
ல்லீல்

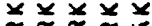

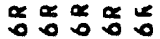
ํํㄴㅇㅠ

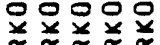

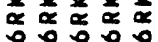
ペ゚゚゚ $\because \therefore$ i

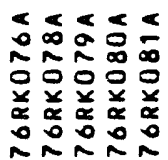

$z z z z=$ 0.000 $i-\bar{v} i$ 웅응응 도 응는의 $\therefore-1 n-$ $<<<<4$

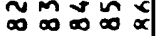
0000

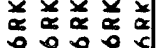




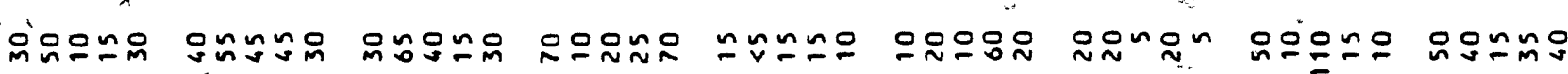

0
$\vdots$
$\vdots$
$\vdots$
$\vdots$

$n \in n n n$

웅으

nnn

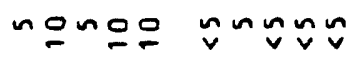

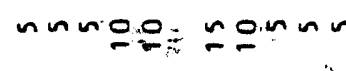

gnonugnonn

$\begin{array}{lll}\approx & 0 \\ 0 & \vdots \\ 0 & \vdots \\ 0 & \vdots & 0\end{array}$

ำกำก ำกนกn กับ

은ํำ

กะกทั

$-5$ (1)

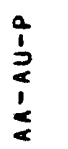

$z z z z \quad z z z z$

22222

$2222 z$

$z 2 z z 2$

$z z 2 z$

$z 2.2 z \cdot 2$

$z 2 z z-z \quad z 2 z 2 z$

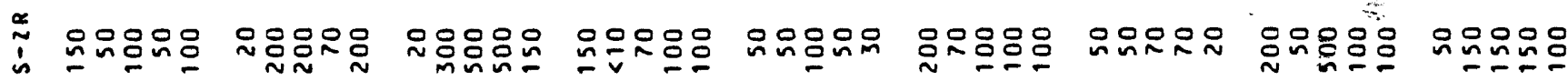

$\infty$

$\underset{⿱ ㇒}{\infty}$

$\grave{i}$

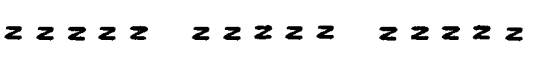

$\stackrel{g}{\tilde{i}}^{22 x}$

$2 \geq 2 \geq 2$

$2 z 2 z$

$z z z z$
-3 $\underset{N}{ }$

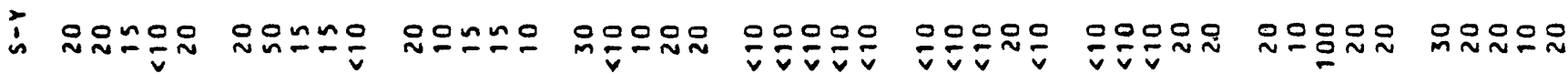

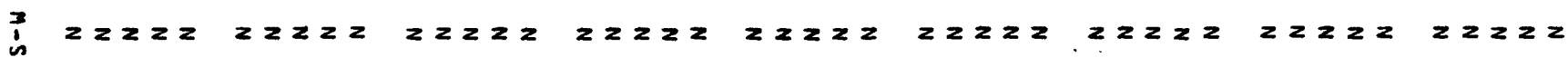

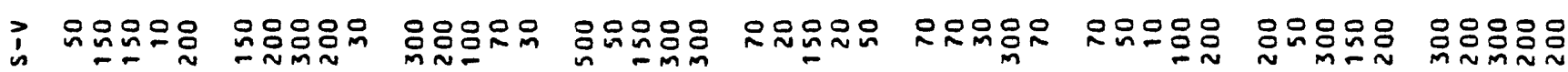
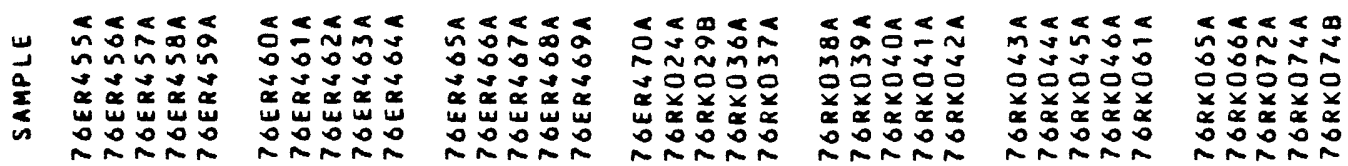

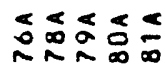
jo 000

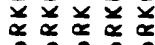




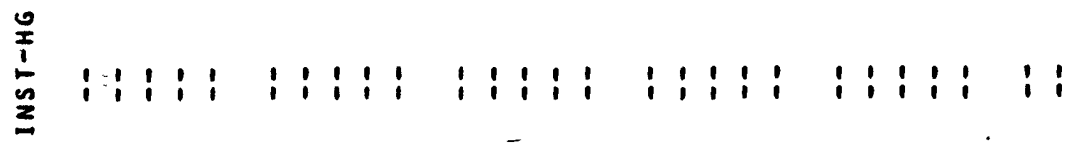

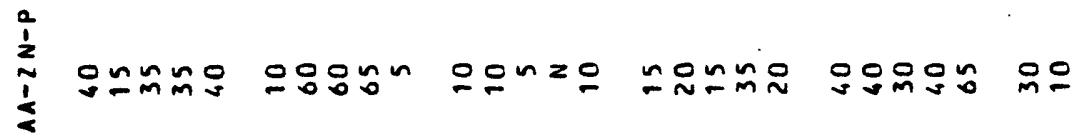

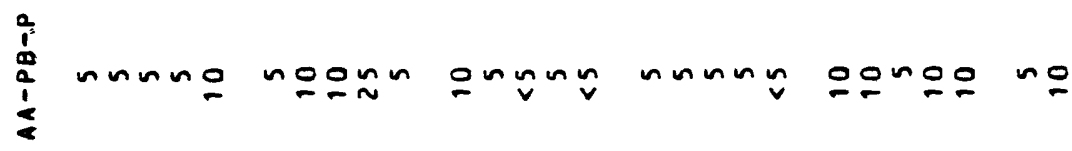

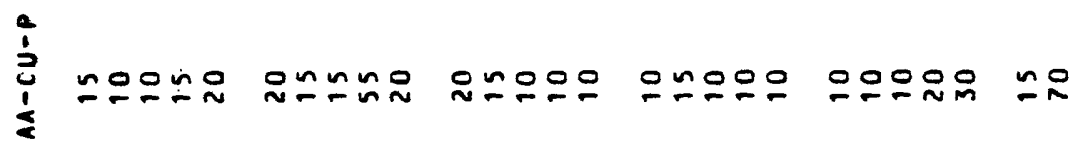
$\vdots$
$\vdots$
$\vdots$
$\vdots$

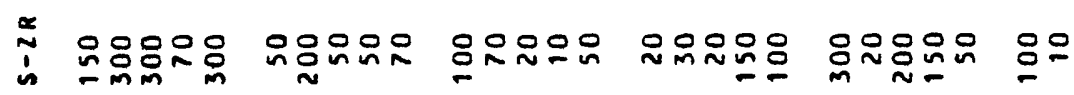

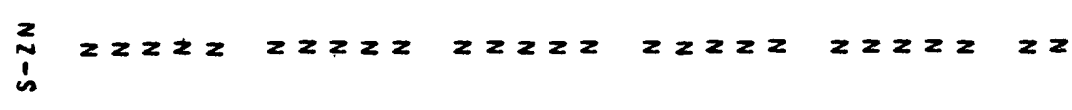

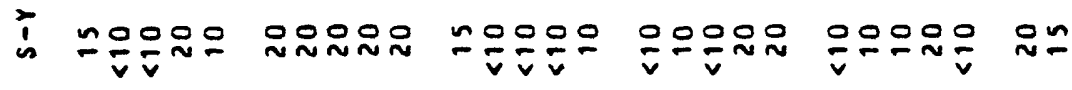

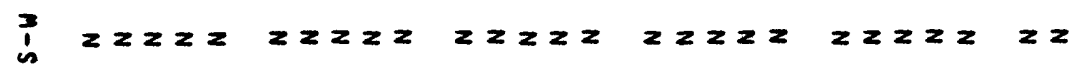

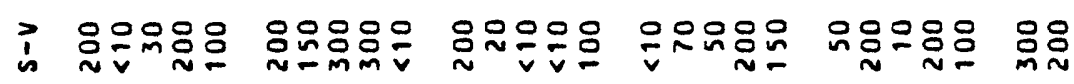
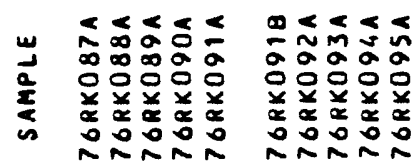

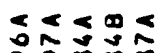

20,

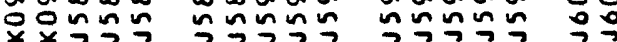

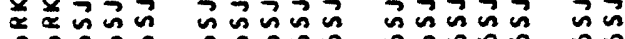

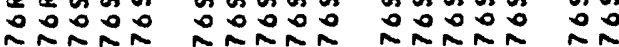




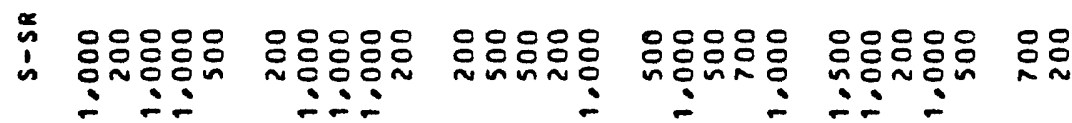
is $\begin{aligned} & z \\ & \text { i }\end{aligned}$

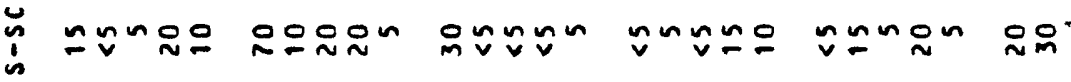

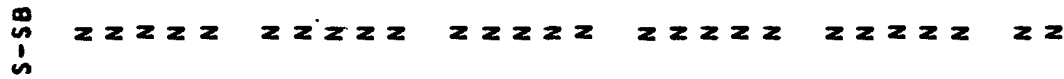

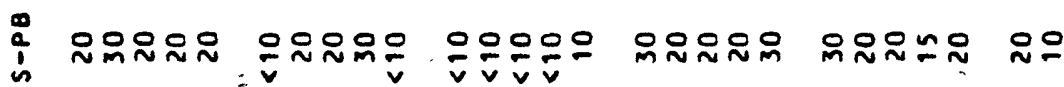

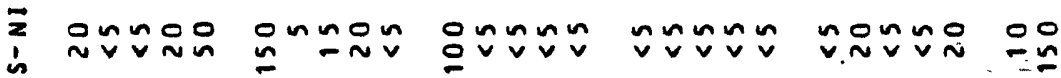

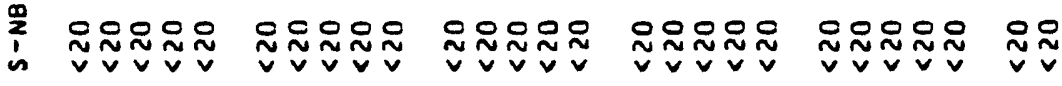

帝

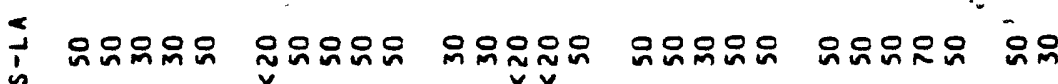

¿ ㅇำท응

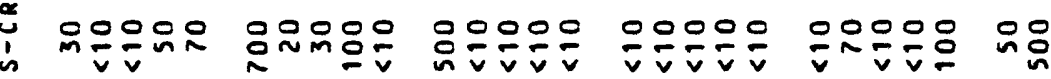

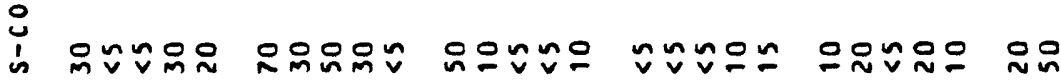
$\dot{\infty}$

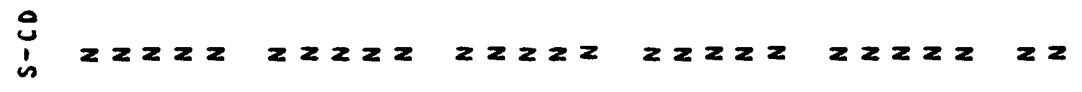

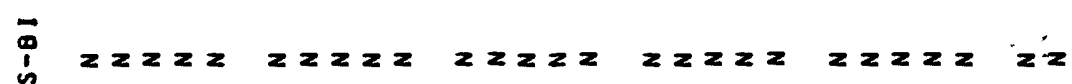
岁

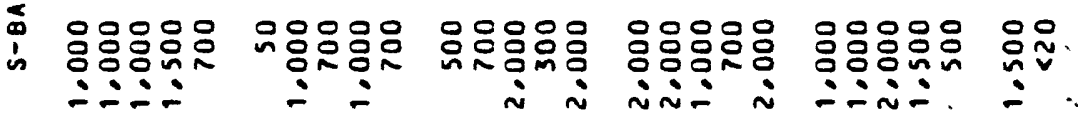

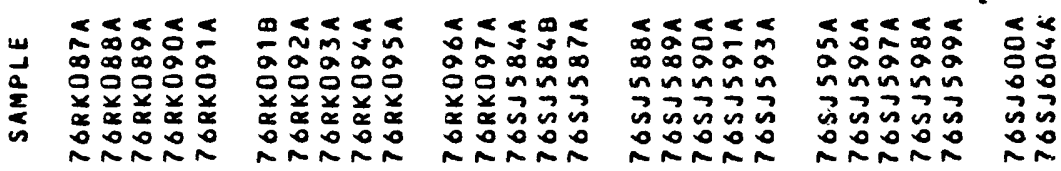

